\title{
Fourier PCA and Robust Tensor Decomposition
}

\author{
Navin Goyal * \\ Santosh Vempala ${ }^{\dagger}$ \\ Ying Xiao $\ddagger$
}

July 1, 2014

\begin{abstract}
Fourier PCA is Principal Component Analysis of a matrix obtained from higher order derivatives of the logarithm of the Fourier transform of a distribution. We make this method algorithmic by developing a tensor decomposition method for a pair of tensors sharing the same vectors in rank-1 decompositions. Our main application is the first provably polynomial-time algorithm for underdetermined ICA, i.e., learning an $n \times m$ matrix $A$ from observations $y=A x$ where $x$ is drawn from an unknown product distribution with arbitrary non-Gaussian components. The number of component distributions $m$ can be arbitrarily higher than the dimension $n$ and the columns of $A$ only need to satisfy a natural and efficiently verifiable nondegeneracy condition. As a second application, we give an alternative algorithm for learning mixtures of spherical Gaussians with linearly independent means. These results also hold in the presence of Gaussian noise.
\end{abstract}

\footnotetext{
*Microsoft Research India

†Schools of Computer Science, Georgia Tech and CMU

¥School of Computer Science, Georgia Tech
} 


\section{Introduction}

Principal Component Analysis [55] is often an "unreasonably effective" heuristic in practice, and some of its effectiveness can be explained rigorously as well (see, e.g., 43.). It consists of computing the eigenvectors of the empirical covariance matrix formed from the data; the eigenvectors turn out to be directions that locally maximize second moments. The following example illustrates the power and limitations of PCA: given random independent points from a rotated cuboid in $\mathbb{R}^{n}$ with distinct axis lengths, PCA will identify the axes of the cuboid and their lengths as the eigenvectors and eigenvalues of the covariance matrix. However, if instead of a rotation, points came from a linear transformation of a cuboid, then PCA does not work.

To handle this and similar hurdles, higher moment extensions of PCA have been developed in the literature e.g., [5, 40, 53, 6, 3, 39, 65, and shown to be provably effective for a wider range of unsupervised learning problems, including special cases of Independent Component Analysis (ICA), Gaussian mixture models, learning latent topic models etc. ICA is the classic signal recovery problem of learning a linear transformation $A$ from i.i.d. samples $x=A s$ where $s \in \mathbb{R}^{n}$ has an unknown product distribution. The example above, namely learning a linearly transformed cuboid, is a special case of this problem. Although PCA fails, one can use it to first apply a transformation (to a sample) that makes the distribution isotropic, i.e., effectively making the distribution a rotation of a cube. At this point, eigenvectors give no further information, but as observed in the signal processing literature [26, 41], directions that locally maximize fourth moments reveal the axes of the cube, and undoing the isotropic transformation yields the axes of the original cuboid. Using this basic idea, Frieze et al. 35] and subsequent papers give provably efficient algorithms assuming that the linear transformation $A$ is full-dimensional and the components of the product distribution differ from one-dimensional Gaussians in their fourth moment. This leaves open the important general case of underdetermined ICA, namely where $A$ is not necessarily square or full-dimensional, i.e., the observations $x$ are projections to a lower-dimensional space; in the case of the cuboid example, we only see samples from an (unknown) projection of a transformed cuboid.

In this paper, we give a polynomial-time algorithm that (a) works for any transformation $A$ provided the columns of the linear transformation satisfy a natural extension of linear independence, (b) does not need the fourth moment assumption, and (c) is robust to Gaussian noise. As far as we know, this is the first polynomial-time algorithm for underdetermined ICA. The central object of our study is a higher derivative tensor of suitable functions of the Fourier transform of the distribution. Our main algorithmic technique is an efficient tensor decomposition method for pairs of tensors that share the same vectors in their respective rank-1 decompositions. We call our general technique Fourier PCA. This is motivated by the fact that in the base case of second derivatives, it reduces to PCA of a reweighted covariance matrix.

As a second application, Fourier PCA gives an alternative algorithm for learning a mixture of spherical Gaussians, under the assumption that the means of the component Gaussians are linearly independent. Hsu and Kakade [39] already gave an algorithm for this problem based on third moments; our algorithm has the benefit of being robust to Gaussian noise.

We now discuss these problems and prior work, then present our results in more detail.

ICA Blind source separation is a fundamental problem in diverse areas ranging from signal processing to neuroscience to machine learning. In this problem, a set of source signals are mixed in an unknown way, and one would like to recover the original signals or understand how they were mixed given the observations of the mixed signals. Perhaps the most influential formalization of this problem is ICA (see the books 26. 41] for comprehensive introductions). In the basic formulation of ICA, one has a random vector $s \in \mathbb{R}^{n}$ (the source signal) whose components are independent random variables with unknown distributions. Let $s^{(1)}, s^{(2)}, \ldots$ be independent samples of $s$. One observes mixed samples $A s^{(1)}, A s^{(2)}, \ldots$ obtained by mixing the components of $s$ by an unknown invertible $n \times n$ mixing matrix A. The goal is to recover $A$ to the extent possible, which would then also give us $s^{(1)}, s^{(2)}, \ldots$, or some approximations thereof. One cannot hope to recover $A$ in case more than one of the $s_{i}$ are Gaussian; in this case any set of orthogonal directions in this subspace would also be consistent with the model. Necessarily, then, all ICA algorithms must require that the component distributions differ from being Gaussian in some fashion.

A number of algorithms have been devised in the ICA community for this problem. The literature is vast and we refer to [26] for a comprehensive account. The ICA problem has been studied rigorously in theoretical 
computer science in several previous papers [35, 54, 9, 12, 5]. All of these algorithms either assume that the component distribution is a very specific one [54, 9], or assume that its kurtosis (fourth cumulant) is bounded away from 0 , in effect assuming that its fourth moment is bounded away from that of a Gaussian. The application of tensor decomposition to ICA has its origins in work by Cardoso [21, and similar ideas were later discovered by Chang [24] in the context of phylogenetic reconstruction and developed further in several works, e.g. Mossel and Roch [53, Anandkumar et al. [3], Hsu and Kakade [39] for various latent variable models. Arora et al. [9] and Belkin et al. [12] show how to make the algorithm resistant to unknown Gaussian noise.

Underdetermined ICA, where the transformation matrix $A$ is not square or invertible (i.e., it includes a projection), is an important general problem and there are a number of algorithms proposed for it in the signal processing literature, many of them quite sophisticated. However, none of them is known to have rigourous guarantees on the sample or time complexity, even for special distributions. See e.g. Chapter 9 of [26] for a review of existing algorithms and identifiability conditions for underdetermined ICA. For example, FOOBI [22, 32] uses fourth-order correlations, and its analysis is done only for the exact setting without analyzing the robustness of the algorithm when applied to a sample, and bounding the sample and time complexity for a desired level of error. In addition, the known sufficient condition for the success of FOOBI is stronger than ours (and more elaborate). We mention two other related papers [27, 2].

Gaussian mixtures Gaussian mixtures are a popular model in statistics. A distribution $F$ in $\mathbb{R}^{n}$ is modeled as a convex combination of unknown Gaussian components. Given i.i.d. samples from $F$, the goal is to learn all its parameters, i.e., the means, covariances and mixing weights of the components. A classical result in statistics says that Gaussian mixtures with distinct parameters are uniquely identifiable, i.e., as the number of samples goes to infinity, there is unique decomposition of $F$ into Gaussian components. It has been established that the sample complexity grows exponentially in $m$, the number of components [14, 13, 42, 51. In a different direction, under assumptions of separable components, a mixture is learnable in time polynomial in all parameters $64,28,58,30,25,19$. . Our work here is motivated by Hsu and Kakade's algorithm [39, which uses a tensor constructed from the first three moments of the distribution and works for a mixture of spherical Gaussians with linearly independent means.

Robust tensor decomposition As a core subroutine for all problems above, we develop a general theory of efficient tensor decompositions for pairs of tensors, which allows us to recover a rank- 1 tensor decomposition from two homogeneous tensor relations. As noted in the literature, such a pair of tensor equations can be obtained from one tensor equation by applying two random vectors to the original equation, losing one in the order of the tensor. Our tensor decomposition "flattens" these tensors to matrices and performs an eigenvalue decomposition. The matrices in question are not Hermitian or even normal, and hence we use more general methods for eigendecomposition (in particular, tensor power iterations cannot be used to find the desired decompositions). The algorithm for tensor decomposition via simultaneous tensor diagonalization is essentially due to Leurgans et al [48; to the best of our knowledge, ours is the first robust analysis. In subsequent work, Bhaskara et al. [15] have outlined a similar robustness analysis with a different application.

\subsection{Results}

We begin with fully determined ICA. Unlike most of the literature on ICA, which employs moments, we do not require that our underlying random variables $s_{i}$ differ from a Gaussian at the fourth moment. In fact, our algorithm can deal with differences from being Gaussian at any moment, though the computational and sample complexities are higher when the differences are at higher moments. We will use cumulants as a notion of difference from being a Gaussian. The cumulant of random variable $x$ at order $r$, denoted by $\operatorname{cum}_{r}(x)$, is the $r^{\text {th }}$ moment with some additional subtractions of polynomials of lower moments. The following is a short statement of our result for fully-determined ICA (i.e. the mixing matrix $A$ is invertible); the full statement appears later as Theorem 4.2. The algorithm takes as input the samples generated according to the ICA model and parameters $\epsilon, \Delta, M, k$ and an estimate of $\sigma_{n}(A)$.

Theorem 1.1. Let $x \in \mathbb{R}^{n}$ be given by an ICA model $x=$ As where $A \in \mathbb{R}^{n \times n}$ columns of $A$ have unit 
norm and let $\sigma_{n}(A)>0$. Suppose that for each $s_{i}$, there exists a $k_{i} \leq k$ such that $\left|\operatorname{cum}_{k_{i}}\left(s_{i}\right)\right| \geq \Delta>0$ and $\mathbb{E}\left(\left|s_{i}\right|^{k}\right) \leq M$. Then, one can recover the columns of $A$ up to signs to $\epsilon$ accuracy in polynomial time using $\operatorname{poly}\left(n^{k^{2}}, M^{k}, 1 / \Delta^{k}, 1 / \sigma_{n}(A)^{k}, 1 / \epsilon\right)$

samples with high probability.

In the simplest setting, roughly speaking, our algorithm computes the covariance matrix of the data reweighted according to a random Fourier coefficient $e^{i u^{T} x}$ where $u \in \mathbb{R}^{n}$ is picked according to a Gaussian distribution. Our ideas are inspired by the work of Yeredor [69], who presented such an algorithm for fully determined ICA (without finite sample guarantees).

The reweighted covariance matrix can also be viewed as the Hessian of the logarithm of the Fourier transform of the distribution. Using this perspective, we extend the method to underdetermined instancesproblems where the apparent number of degrees of freedom seems higher than the measurement system can uniquely fix, by studying higher derivative tensors of the Fourier transform. The use of Fourier weights has the added advantage that the resulting quantities always exist (this is the same phenomenon that for a probability distribution the characteristic function always exists, but the moment generating function may not) and thus works for all random variables and not just in the case of having all finite moments.

We then extend this to the setting where the source signal $s$ has more components than the number of measurements (Section 6); recall that in this case, the transformation $A$ is a map to a lower-dimensional space. Finding provably efficient algorithms for underdetermined ICA has been an open problem. Tensor decomposition techniques, such as power iteration, which are known to work in the fully determined case cannot possibly generalize to the underdetermined case [4, as they require linear independence of the columns of $A$, which means that they can handle at most $n$ source variables.

Our approach is based on tensor decomposition, usually defined as follows: given a tensor $T \in \mathbb{R}^{n \times \cdots \times n}$ which has the following rank-1 expansion:

$$
T=\sum_{i=1}^{m} \mu_{i} A_{i} \otimes \cdots \otimes A_{i}
$$

compute the vectors $A_{i} \in \mathbb{R}^{n}$. Here $\otimes$ denotes the usual outer product so that entry-wise $[v \otimes \cdots \otimes v]_{i_{1}, \ldots, i_{r}}=$ $\left.v_{i_{1}} \cdots v_{i_{r}}\right)$. Our main idea here is that we do not attempt to decompose a single tensor into its rank-1 components. This is an NP-hard problem in general, and to make it tractable, previous work uses additional informaton and structural assumptions, which do not hold in the underdetermined setting or place strong restrictions on how large $m$ can be as a function of $n$. Instead, we consider two tensors which share the same rank-1 components and compose the tensors in a specific way, thereby extracting the desired rank-1 components. In the following vec $\left(A_{i}^{\otimes d / 2}\right)$ denotes the tensor $A_{i}^{\otimes d / 2}$ flattened into a vector. The algorithm's input consists of: tensors $T_{\mu}, T_{\lambda}$, and parameters $n, m, d, \Delta, \epsilon$ as explained in the following theorem.

Theorem 1.2 (Tensor decomposition). Let $A$ be an $n \times m$ matrix with $m>n$ and columns with unit norm, and let $T_{\mu}, T_{\lambda} \in \mathbb{R}^{n \times \cdots \times n}$ be order $d$ tensors such that $d \in 2 \mathbb{N}$ and

$$
T_{\mu}=\sum_{i=1}^{m} \mu_{i} A_{i}^{\otimes d} \quad T_{\lambda}=\sum_{i=1}^{m} \lambda_{i} A_{i}^{\otimes d}
$$

where $\operatorname{vec}\left(A_{i}^{\otimes d / 2}\right)$ are linearly independent, $\mu_{i}, \lambda_{i} \neq 0$ and $\left|\frac{\mu_{i}}{\lambda_{i}}-\frac{\mu_{j}}{\lambda_{j}}\right|>\Delta$ for all $i, j$ and $\Delta>0$. Then, algorithm TensorDecomposition $\left(T_{\mu}, T_{\lambda}\right)$ outputs vectors $A_{1}^{\prime}, \ldots, A_{m}^{\prime}$ with the following property. There is a permutation $\pi:[m] \rightarrow[m]$ and signs $\alpha:[m] \rightarrow\{-1,1\}$ such that for $i \in[m]$ we have

$$
\left\|\alpha_{i} A_{\pi(i)}^{\prime}-A_{i}\right\|_{2} \leq \epsilon
$$

The running time is poly $\left(n^{d}, \frac{1}{\epsilon}, \frac{1}{\Delta}, \frac{1}{\sigma_{\min }\left(A^{\odot d / 2}\right)}\right)$. 
The polynomial in the running time above can be made explicit. It basically comes from the time complexity of SVD and eigenvector decomposition of diagonalizable matrices. We note that in contrast to previous work on tensor decompositions [37, 33, 23, 59, our method has provable finite sample guarantees. We give a robust version of the above, stated as Theorem 5.4

To apply this to underdetermined ICA, we form tensors associated with the ICA distribution as inputs to our pairwise tensor decomposition algorithm. The particular tensors that we use are the derivative tensors of the second characteristic function evaluated at random points.

Our algorithm can handle unknown Gaussian noise. The ICA model with Gaussian noise is given by

$$
x=A s+\eta,
$$

where $\eta \sim N(0, \Sigma)$ is independent Gaussian noise with unknown general covariance matrix $\Sigma \in \mathbb{R}^{n \times n}$. Also, our result does not need full independence of the $s_{i}$, it is sufficient to have $d$-wise independence. The following is a short statement of our result for underdetermined ICA; the full statement appears later as Theorem 6.8 (but without noise). Its extension to handling Gaussian noise is in Sec. 4.8. The input to the algorithms, apart from the samples generated according to the unknown noisy underdetermined ICA model, consists of several parameters whose meaning will be clear in the theorem statement below: A tensor order parameter $d$, number of signals $m$, accuracy parameter $\epsilon$, confidence parameter $\delta$, bounds on moments and cumulants $M$ and $\Delta$, an estimate of the conditioning parameter $\sigma_{m}$, and moment order $k$. The notation $A^{\odot d}$ used below is explained in the preliminaries section; briefly, it's a $n^{d} \times m$ matrix with each column obtained by flattening $A_{i}^{\otimes d}$ into a vector.

Theorem 1.3. Let $x \in \mathbb{R}^{n}$ be given by an underdetermined ICA model with unknown Gaussian noise $x=A s+\eta$ where $A \in \mathbb{R}^{n \times m}$ with unit norm columns and the covariance matrix $\Sigma \in \mathbb{R}^{n \times n}$ are unknown. Let $d \in 2 \mathbb{N}$ be such that $\sigma_{m}\left(A^{\odot d / 2}\right)>0$. Let $M_{k}, M_{d}, M_{2 d}$ and $k>d$ be such that for each $s_{i}$, there is a $k_{i}$ satisfying $d<k_{i}<k$ and $\left|\operatorname{cum}_{k_{i}}\left(s_{i}\right)\right| \geq \Delta$, and $\mathbb{E}\left(\left|s_{i}\right|^{k_{i}}\right), \mathbb{E}\left(\sigma_{1}(\Sigma)^{k}\right) \leq M_{k}, \mathbb{E}\left(\left|s_{i}\right|^{d}\right) \leq M_{d}$, and $\mathbb{E}\left(\left|s_{i}\right|^{2 d}\right) \leq M_{2 d}$. Then one can recover the columns of $A$ up to $\epsilon$ accuracy in 2-norm and up to the sign using poly $\left(m^{k}, M_{d}^{k}, M_{2 d}, 1 / \Delta, 1 / \sigma_{m}\left(A^{\odot d / 2}\right)^{k}, 1 / \epsilon, 1 / \sigma^{k}\right)$ samples and with similar polynomial time complexity with probability at least $3 / 4$, where $0<\sigma<\frac{\Delta}{M_{k}} \operatorname{poly}\left(\sigma_{m}\left(m^{k}, A^{\odot d / 2}\right)^{k}, 1 / k^{k}\right)$.

The probability of success of the algorithm can be boosted from $3 / 4$ to $1-\delta$ for any $\delta>0$ by taking $O(\log (1 / \delta))$ independent runs of the algorithm and using an adaptation of the "median" trick (see e.g., Thm 2.8 in 49 ). To our knowledge, this is the first polynomial-time algorithm for underdetermined ICA with provable finite sample guarantees. It works under mild assumptions on the input distribution and nondegeneracy assumptions on the mixing matrix $A$. The assumption says that the columns of the matrix when tensored up individually are linearly independent. For example, with $d=4$, suppose that every $s_{i}$ differs from a Gaussian in the fifth or higher moment by $\Delta$, then we can recover all the components as long as vec $\left(A_{i} A_{i}^{T}\right)$ are linearly independent. Thus, the number of components that can be recovered can be as high as $m=n(n+1) / 2$. Clearly, this is a weakening of the standard assumption that the columns of $A$ are linearly independent. This assumption can be regarded as a certain incoherence type assumption. Moreover, in a sense it's a necessary and sufficient condition: the ICA problem is solvable for matrix $A$ if and only if any two columns are linear independent [26, and this turns out to be equivalent to the existence of a finite $d$ such that $A^{\odot d}$ has full column rank. A well-known condition in the literature on tensor decomposition is Kruskal's condition [45]. Unlike that condition it is easy to check if a matrix satisfies our assumption (for

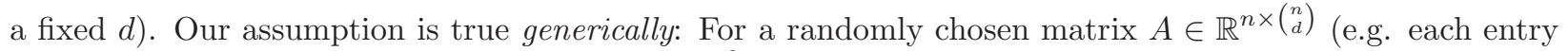
being i.i.d. standard Gaussian), we have $\sigma_{\min }\left(A^{\odot d}\right)>0$ with probability 1. In a similar vein, for a randomly chosen matrix $A \in \mathbb{R}^{n \times\left(\begin{array}{l}n \\ d\end{array}\right)}$ its condition number is close to 1 with high probability; see Theorem 9.2 for a precise statement and proof. Moreover, our assumption is robust also in the smoothed sense [7]: If we

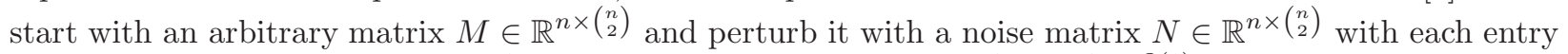
independently chosen from $N\left(0, \sigma^{2}\right)$, then we have $\sigma_{\min }\left((M+N)^{\odot 2}\right)=\sigma^{2} / n^{O(1)}$ with probability at least $1-1 / n^{\Omega(1)}$, and a similar generalization holds for higher powers. This follows from a simple application of the anti-concentration properties of polynomials in independent random variables; see [7] for a proof. See also [15]. 
As in the fully-determined ICA setting, we require that our random variables have some cumulant different from a Gaussian. One aspect of our result is that using the $d^{\text {th }}$ derivative, one loses the ability to detect non-Gaussian cumulants at order $d$ and lower; on the other hand, a theorem of Marcinkiewicz [50] implies that this does not cause a problem.

Theorem 1.4 (Marcinkiewicz). Suppose that random variable $x \in \mathbb{R}$ has only a finite number of nonzero cumulants, then $x$ is distributed according to a Gaussian, and every cumulant of order greater than 2 vanishes.

Thus, even if we miss the difference in cumulants at order $i \leq d$, there is some higher order cumulant which is nonzero, and hence non-Gaussian. Note also that for many specific instances of the ICA problem studied in the literature, all cumulants differ from those of a Gaussian [35, 54, 9].

We remark that apart from direct practical interest of ICA in signal recovery, recently some new applications of ICA as an algorithmic primitive have been discovered. Anderson et al. [8] reduce some special cases of the problem of learning a convex body (coming from a class of convex bodies such as simplices), given uniformly distributed samples from the body, to fully-determined ICA. Anderson et al. 7 solve the problem of learning Gaussian mixture models in regimes for which there were previously no efficient algorithms known. This is done by reduction to underdetermined ICA using the results of our paper.

Our final result applies the same method to learning mixtures of spherical Gaussians (see the full version). Using Fourier PCA, we recover the result of Hsu and Kakade [39, and extend it to the setting of noisy mixtures, where the noise itself is an unknown arbitrary Gaussian. Our result can be viewed as saying that reweighted PCA gives an alternative algorithm for learning such mixtures.

Theorem 1.5. Fourier PCA for Mixtures applied to a mixture of $k<n$ spherical Gaussians $N\left(\mu_{i}, \sigma_{i}^{2} I_{n}\right)$ recovers the parameters of the mixture to desired accuracy $\epsilon$ using time and samples polynomial in $k, n, 1 / \epsilon$ with high probability, assuming that the means $\mu_{i}$ are linearly independent.

Thus, overall, our contributions can be viewed as two-fold. The first part is a robust, efficient tensor decomposition technique. The second is the analysis of the spectra of matrices/tensors arising from Fourier derivatives. In particular, showing that the eigenvalue gaps are significant based on anticoncentration of polynomials in Gaussian space; and that these matrices, even when obtained from samples, remain diagonalizable.

\section{Preliminaries}

For positive integer $n$, the set $\{1, \ldots, n\}$ is denoted by $[n]$. The set of positive even numbers is denoted by $2 \mathbb{N}$.

We assume for simplicity and without real loss of generality that $\mathbb{E}\left(s_{j}\right)=0$ for all $j$. We can ensure this by working with samples $x^{i}-\bar{x}$ instead of the original samples $x^{i}$ (here $\bar{x}$ is the empirical average of the samples). There is a slight loss of generality because using $\bar{x}$ (as opposed to using $\mathbb{E}(() x)$ ) introduces small errors. These errors can be analysed along with the rest of the errors and do not introduce any new difficulties.

Probability. For a random variable $x \in \mathbb{R}^{n}$ and $u \in \mathbb{R}^{n}$, its characteristic function $\phi: \mathbb{R} \rightarrow \mathbb{C}$ is defined by $\phi_{x}(u)=\mathbb{E}_{x}\left(e^{i u^{T} x}\right)$. Unlike the moment generating function, the characteristic function is well-defined even for random variables without all moments finite. The second characteristic function of $x$ is defined by $\psi_{x}(u):=\log \phi_{x}(u)$, where we take that branch of the complex logarithm that makes $\psi(0)=0$. In addition to random variable $x$ above we will also consider random variable $s \in \mathbb{R}^{m}$ related to $x$ via $x=A s$ for $A \in \mathbb{R}^{n \times m}$ and the functions associated with it: the characteristic function $\phi_{s}(t)=\mathbb{E}_{s}\left(e^{i t^{T} s}\right)$ and the second characteristic function $\psi_{s}(t)=\log \phi_{s}(t)$.

Let $\mu_{j}:=\mathbb{E}\left(x^{j}\right)$. Cumulants of $x$ are polynomials in the moments of $x$ which we now define. For $j \geq 1$, the $j$ th cumulant is denoted $\operatorname{cum}_{j}(x)$. Some examples: $\operatorname{cum}_{1}(x)=\mu_{1}, \operatorname{cum}_{2}(x)=\mu_{2}-\mu_{1}^{2}, \operatorname{cum}_{3}(x)=$ $\mu_{3}-3 \mu_{2} \mu_{1}+2 \mu_{1}^{3}$. As can be seen from these examples the first two cumulants are the same as the expectation 
and the variance, resp. Cumulants have the property that for two independent r.v.s $x, y$ we have $\operatorname{cum}_{j}(x+y)=$ $\operatorname{cum}_{j}(x)+\operatorname{cum}_{j}(y)$ (assuming that the first $j$ moments exist for both $x$ and $y$ ). The first two cumulants of the standard Gaussian distribution have value 0 and 1, and all subsequent cumulants have value 0 . Since ICA is not possible if all the independent component distributions are Gaussians, we need some measure of distance from the Gaussians of the component distributions. A convenient measure turns out to be the distance from 0 (i.e. the absolute value) of the third or higher cumulants. If all the moments of $x$ exist, then the second characteristic function admits a Taylor expansion in terms of cumulants

$$
\psi_{x}(u)=\sum_{j \geq 1} \operatorname{cum}_{j}(x) \frac{(i u)^{j}}{j !} .
$$

This can also be used to define cumulants of all orders.

Matrices. For a vector $\mu=\left(\mu_{1}, \ldots, \mu_{n}\right)$, let $\operatorname{diag}(\mu)$ and $\operatorname{diag}\left(\mu_{j}\right)$, where $j$ is an index variable, denote the $n \times n$ diagonal matrix with the diagonal entries given by the components of $\mu$. The singular values of an $m \times n$ matrix will always be ordered in the decreasing order: $\sigma_{1} \geq \sigma_{2} \geq \ldots \geq \sigma_{\min (m, n)}$. Our matrices will often have rank $m$, and thus the non-zero singular values will often, but not always, be $\sigma_{1}, \ldots, \sigma_{m}$. The span of the columns vectors of a matrix $A$ will be denoted colspan $(A)$. The columns of a matrix $A$ are denoted $A_{1}, A_{2}, \ldots$ The potentially ambiguous but convenient notation $A_{i}^{T}$ means $\left(A_{i}\right)^{T}$. The condition number of a matrix $A$ is $\kappa(A):=\sigma_{\max }(A) / \sigma_{\min }(A)$, where $\sigma_{\max }(A):=\sigma_{1}(A)$ and $\sigma_{\min }(A):=\sigma_{\min (m, n)}(A)$.

Tensors and tensor decomposition. Here we introduce various tensor notions that we need; these are discussed in detail in the review paper [44. An order $d$ tensor $T$ is an array indexed by $d$ indices each with $n$ values (e.g., when $d=2$, then $T$ is simply a matrix of size $n \times n$ ). Thus, it has $n^{d}$ entries. Tensors considered in this paper are symmetric, i.e. $T_{i_{1}, \ldots, i_{d}}$ is invariant under permutations of $i_{1}, \ldots, i_{d}$. In the sequel we will generally not explicitly mention that our tensors are symmetric. We also note that symmetry of tensors is not essential for our results but for our application to ICA it suffices to look at only symmetric tensors and the results generalize easily to the general case, but at the cost of additional notaton.

We can also view a tensor as a degree- $d$ homogeneous form over vectors $u \in \mathbb{R}^{n}$ defined by $T(u, \ldots, u)=$ $\sum_{i_{1}, \ldots, i_{d}} T_{i_{1}, \ldots, i_{d}} u_{i_{1}} \cdots u_{i_{d}}$. This is in analogy with matrices, where every matrix $A$ defines a quadratic form, $u^{T} A u=A(u, u)=\sum_{i, j} A_{i, j} u_{i} u_{j}$. We use the outer product notation

$$
v^{\otimes d}=\underbrace{v \otimes \cdots \otimes v}_{d \text { copies }},
$$

where entrywise we have $[v \otimes \cdots \otimes v]_{j_{1}, \ldots, j_{d}}=v_{j_{1}} \cdots v_{j_{d}}$. A (symmetric) rank-1 decomposition of a tensor $T_{\mu}$ is defined by

$$
T_{\mu}=\sum_{i=1}^{m} \mu_{i} A_{i}^{\otimes d}
$$

where the $\mu_{i} \in \mathbb{R}$ are nonzero and the $A_{i} \in \mathbb{R}^{n}$ are vectors which are not multiples of each other. Such a decomposition always exists for all symmetric tensors with $m<n^{d}$ (better bounds are known but we won't need them). For example, for a symmetric matrix, by the spectral theorem we have

$$
M=\sum_{i=1}^{n} \lambda_{i} v_{i} \otimes v_{i}
$$

We will use the notion of flattening of tensors. Instead of giving a formal definition it's more illuminating to give examples. E.g. for $d=4$, construct a bijection $\tau:\left[n^{2}\right] \rightarrow[n] \times[n]$ as $\tau(k)=(\lfloor k / n\rfloor, k-\lfloor k / n\rfloor)$ and $\tau^{-1}(i, j)=n i+j$. We then define a packing of a matrix $B \in \mathbb{R}^{n \times n}$ to a vector $p \in \mathbb{R}^{n^{2}}$ by $B_{\tau(k)}=p_{k}$. For convenience we will say that $B=\tau(p)$ and $p=\tau^{-1}(B)$. We also define a packing of $T \in \mathbb{R}^{n \times n \times n \times n}$ 
to a matrix $M \in \mathbb{R}^{n^{2} \times n^{2}}$ by $M_{a, b}=T_{\tau(a), \tau(b)}$, for $a, b \in\left[n^{2}\right]$. Note that $M$ is symmetric because $T$ is symmetric with respect to all permutations of indices: $M_{a, b}=T_{\tau(a), \tau(b)}=T_{\tau(b), \tau(a)}=M_{b, a}$. The definition of $\tau$ depends on the dimensions and order of the tensor and what it's being flattened into; this will be clear from the context and will not be further elaborated upon. Finally, to simplify the notation, we will employ the Khatri-Rao power of a matrix: $A^{\odot d}:=\left[\operatorname{vec}\left(A_{1}^{\otimes d}\right)\left|\operatorname{vec}\left(A_{2}^{\otimes d}\right)\right| \ldots \mid \operatorname{vec}\left(A_{m}^{\otimes d}\right)\right]$, where recall that vec $(T)$ for a tensor $T$ is a flattening of $T$, i.e. we arrange the entries of $T$ in a single column vector.

Derivatives. For $g: \mathbb{R}^{n} \rightarrow \mathbb{R}$ we will use abbreviation $\partial_{u_{i}} g\left(u_{1}, \ldots, u_{n}\right)$ for $\frac{\partial g\left(u_{1}, \ldots, u_{n}\right)}{\partial u_{i}}$; when the variables are clear from the context, we will further shorten this to $\partial_{i} g$. Similarly, $\partial_{i_{1}, \ldots, i_{k}} g$ denotes $\partial_{i_{1}}\left(\ldots\left(\partial_{i_{k}} g\right) \ldots\right)$, and for multiset $S=\left\{i_{1}, \ldots, i_{k}\right\}$, this will also be denoted by $\partial_{S} g$, which makes sense because $\partial_{i_{1}, \ldots, i_{k}} g$ is invariant under reorderings of $i_{1}, \ldots, i_{k}$. We will not use any special notation for multisets; what is meant will be clear from the context.

$D_{u} g(u)$ denotes the gradient vector $\left(\partial_{u_{1}} g(u), \ldots, \partial_{u_{n}} g(u)\right)$, and $D_{u}^{2} g(u)$ denotes the Hessian matrix $\left[\partial_{u_{i}} \partial_{u_{j}} g(u)\right]_{i j}$. More generally, $D_{u}^{d} g(u)$ denotes the order $d$ derivative tensor given by $\left[D_{u}^{d} g(u)\right]_{i_{1}, \ldots, i_{d}}=$ $\partial_{u_{i_{1}}} \ldots \partial_{u_{i_{d}}} g(u)$.

Derivatives and linear transforms. We are particularly interested in how the derivative tensor changes under linear transform of the arguments. We state things over the real field, but everything carries over to the complex field as well. Let $g: \mathbb{R}^{n} \rightarrow \mathbb{R}$ and $f: \mathbb{R}^{m} \rightarrow \mathbb{R}$ be two functions such that all the derivatives that we consider below exist. Let $A \in \mathbb{R}^{n \times m}$ and let variables $t \in \mathbb{R}^{m}$ and $u \in \mathbb{R}^{n}$ be related by linear relation $t=A^{T} u$, and let the function $f$ and $g$ be related by $g(u)=f\left(A^{T} u\right)=f(t)$. Then for $j \in[n]$

$$
\begin{aligned}
\partial_{u_{j}} g(u) & =\partial_{u_{j}} f\left(\left(A_{1}\right)^{T} u, \ldots,\left(A_{m}\right)^{T} u\right) \\
& =\frac{\partial\left(A_{1}\right)^{T} u}{\partial u_{j}} \partial_{t_{1}} f(t)+\ldots+\frac{\partial\left(A_{m}\right)^{T} u}{\partial u_{j}} \partial_{t_{m}} f(t) \\
& =A_{j 1} \partial_{t_{1}} f(t)+\ldots+A_{j m} \partial_{t_{m}} f(t) \\
& =\sum_{k \in[m]} A_{j k} \partial_{t_{k}} f(t) .
\end{aligned}
$$

Applying the previous eqution twice for $i, j \in[n]$ gives

$$
\begin{aligned}
\partial_{u_{i}} \partial_{u_{j}} g(u) & =\partial_{u_{i}}\left(\sum_{k \in[m]} A_{j k} \partial_{t_{k}} f(t)\right) \\
& =\sum_{k \in[m]} A_{j k} \partial_{t_{k}}\left(\partial_{u_{i}} f(t)\right) \\
& =\sum_{k \in[m]} A_{j k} \sum_{\ell \in[m]} A_{i l} \partial_{t_{k}} \partial_{t_{\ell}} f(t) \\
& =\sum_{\ell, k \in[m]} A_{i \ell} A_{j k} \partial_{t_{\ell}} \partial_{t_{k}} f(t),
\end{aligned}
$$

and applying it four times for $i_{1}, i_{2}, i_{3}, i_{4} \in[n]$ gives

$$
\partial_{u_{i_{1}}} \partial_{u_{i_{2}}} \partial_{u_{i_{3}}} \partial_{u_{i_{4}}} g(u)=\sum_{k_{1}, k_{2}, k_{3}, k_{4} \in[m]} A_{i_{1} k_{1}} A_{i_{2} k_{2}} A_{i_{3} k_{3}} A_{i_{4} k_{4}} \partial_{t_{k_{1}}} \partial_{t_{k_{2}}} \partial_{t_{k_{3}}} \partial_{t_{k_{4}}} f(t) .
$$

This can be written more compactly as a matrix equation

$$
D_{u}^{4} g(u)=A^{\otimes 2}\left(D_{t}^{4} f(t)\right)\left(A^{\otimes 2}\right)^{T},
$$


where we interpret both $D_{u}^{4} g(u)$ and $D_{t}^{4} f(t)$ as appropriately flattened into matrices.

A useful special case of this occurs when $f$ has the property that $\partial_{t_{i}} \partial_{t_{j}} f(t)=0$ whenever $i \neq j$. In this case (2) can be rewritten as

$$
\partial_{u_{i_{1}}} \partial_{u_{i_{2}}} \partial_{u_{i_{3}}} \partial_{u_{i_{4}}} g(u)=\sum_{k \in[m]} A_{i_{1} k} A_{i_{2} k} A_{i_{3} k} A_{i_{4} k} \partial_{t_{k}}^{4} f(t)
$$

and in matrix notation

$$
D_{u}^{4} g(u)=A^{\odot 2} \operatorname{diag}\left(\partial_{t_{1}}^{4} f(t), \ldots, \partial_{t_{m}}^{4} f(t)\right)\left(A^{\odot 2}\right)^{T},
$$

where again we interpret $D_{u}^{4} g(u)$ as flattened into a matrix.

The previous equations readily generalize to higher derivatives. For $d \geq 1$, interpreting the tensors $D_{u}^{2 d} g(u)$ and $D_{t}^{2 d} f(t)$ as flattened into matrices, we have

$$
D_{u}^{2 d} g(u)=A^{\otimes d}\left(D_{t}^{2 d} f(t)\right)\left(A^{\otimes d}\right)^{T},
$$

and if $f$ has the property that $\partial_{t_{i}} \partial_{t_{j}} f(t)=0$ whenever $i \neq j$ then

$$
D_{u}^{2 d} g(u)=A^{\odot d} \operatorname{diag}\left(\partial_{t_{1}}^{2 d} f(t), \ldots, \partial_{t_{m}}^{2 d} f(t)\right)\left(A^{\odot d}\right)^{T} .
$$

In our applications we will need to use the above equations for the case when $g(u)=\psi_{x}(u)$ and $f(t)=$ $\psi_{s}(t)$ where these notions were defined at the beginning of this section. The above equations then become

$$
D_{u}^{2 d} \psi_{x}(u)=A^{\otimes d}\left(D_{t}^{2 d} \psi_{s}(t)\right)\left(A^{\otimes d}\right)^{T} .
$$

In the special case when the components of $s$ are indpendent we have $f(t)=\log \mathbb{E}\left(e^{i t_{1} s_{1}}\right)+\ldots+$ $\log \mathbb{E}\left(e^{i t_{m} s_{m}}\right)$ and so we have the property $\partial_{t_{i}} \partial_{t_{j}} \psi_{s}(t)=0$ whenever $i \neq j$ and this gives

$$
D_{u}^{2 d} \psi_{x}(u)=A^{\odot d} \operatorname{diag}\left(\partial_{t_{1}}^{2 d} \psi_{s}(t), \ldots, \partial_{t_{1}}^{2 d} \psi_{s}(t)\right)\left(A^{\odot d}\right)^{T} .
$$

\section{Algorithms}

In this section, we present our main new algorithms and outline their analysis. For the reader's convenience, we will restate these algorithms in the sections where their analysis appears. As mentioned in the introduction, our ICA algorithm is based on a certain tensor decocmposition algorithm.

\subsection{Tensor decomposition}

A fundamental result of linear algebra is that every symmetric matrix has a spectral decomposition, which allows us to write it as the sum of outer products of vectors: $A=\sum_{i=1}^{n} \lambda_{i} v_{i} v_{i}^{T}$, and such representations are efficiently computable. Our goal, in analogy with spectral decomposition for matrices, is to recover (symmetric) rank-1 decompositions of tensors. Unfortunately, there are no known algorithms with provable guarantees when $m>n$, and in fact this problem is NP-hard in general [18, 38. It is an open research question to characterize, or even give interesting sufficient conditions, for when a rank-1 decomposition of a tensor $T$ as in (11) is unique and computationally tractable. For the case $d=2$, a necessary and sufficient condition for uniqueness is that the eigenvalues of $T$ are distinct. Indeed, when eigenvalues repeat, rotations of the $A_{i}$ in the degenerate eigensubspaces with repeated eigenvalues lead to the same matrix $M$.

For $d>2$, if the $A_{i}$ are orthogonal, then the expansion in (11) is unique and can be computed efficiently. The algorithm is power iteration that recovers one $A_{i}$ at a time (see e.g. [5]). The requirement that the $A_{i}$ are orthogonal is necessary for this algorithm, but if one also has access to the order-2 tensor (i.e., matrix) in addition, $M=\sum_{i=1}^{m} A_{i} \otimes A_{i}$, and the $A_{i}$ are linearly indepenent, then one can arrange for the orthogonality of the $A_{i}$ by a suitable linear transformation. However, the fundamental limitation remains that we must take $m \leq n$ simply because we can not have more than $n$ orthogonal vectors in $\mathbb{R}^{n}$. 
Here we consider a modified setting where we are allowed some additional information: suppose we have access to two tensors, both of order $d$, which share the same rank- 1 components, but have different coefficients:

$$
T_{\mu}=\sum_{i=1}^{m} \mu_{i} A_{i}^{\otimes d}, \quad T_{\lambda}=\sum_{i=1}^{m} \lambda_{i} A_{i}^{\otimes d} .
$$

Given such a pair of tensors $T_{\mu}$ and $T_{\lambda}$, can we recover the rank-1 components $A_{i}$ ?

We answer this question in the affirmative for even orders $d \in 2 \mathbb{N}$, and give a provably good algorithm for this problem assuming that the ratios $\mu_{i} / \lambda_{i}$ are distinct. Additionally, we assume that the $A_{i}$ are not scalar multiples of each other, a necessary assumption. We make this quantitative via the $m^{\text {th }}$ singular value of the matrix with columns given by $A_{i}^{\odot d / 2}$. form:

Our algorithm works by flattening tensors $T_{\mu}$ and $T_{\lambda}$ into matrices $M_{\mu}$ and $M_{\lambda}$ which have the following

$$
M_{\mu}=\left(A^{\odot d / 2}\right) \operatorname{diag}\left(\mu_{i}\right)\left(A^{\odot d / 2}\right)^{T}, \quad M_{\lambda}=\left(A^{\odot d / 2}\right) \operatorname{diag}\left(\lambda_{i}\right)\left(A^{\odot d / 2}\right)^{T} .
$$

Taking the product $M_{\mu} M_{\lambda}^{-1}$ yields a matrix whose eigenvectors are the columns of $A^{\odot d / 2}$ and whose eigenvalues are $\mu_{i} / \lambda_{i}$ :

$$
\begin{aligned}
M_{\mu} M_{\lambda}^{-1} & =\left(A^{\odot d / 2}\right) \operatorname{diag}\left(\mu_{i}\right)\left(A^{\odot d / 2}\right)^{T}\left(\left(A^{\odot d / 2}\right)^{T}\right)^{-1} \operatorname{diag}\left(\lambda_{i}\right)^{-1}\left(A^{\odot d / 2}\right)^{-1} \\
& =\left(A^{\odot d / 2}\right) \operatorname{diag}\left(\mu_{i} / \lambda_{i}\right)\left(A^{\odot d / 2}\right)^{-1} .
\end{aligned}
$$

Actually, for the last equation to make sense one needs that $A^{\odot d / 2}$ be invertible which will generally not be the case as $A^{\odot d / 2}$ is not even a square matrix in general. We handle this by restricting $M_{\mu}$ and $M_{\lambda}$ to linear transform from their pre-image to the image. This is the reason for introducing matrix $W$ in algorithm Diagonalize $\left(M_{\mu}, M_{\lambda}\right)$ below.

The main algorithm below is Tensor Decomposition $\left(T_{\mu}, T_{\lambda}\right)$ which flattens the tensors and calls subroutine Diagonalize $\left(M_{\mu}, M_{\lambda}\right)$ to get estimates of the $A_{i}^{\odot d / 2}$, and from this information recovers the $A_{i}$ themselves. In our application it will be the case that $\mu, \lambda \in \mathbb{C}^{m}$ and $A_{i} \in \mathbb{R}^{n}$. The discussion below is tailored to this situation; the other interesting cases where everything is real or everything is complex can also be dealt with with minor modifications.

$\operatorname{Diagonalize}\left(M_{\mu}, M_{\lambda}\right)$

1. Compute the SVD of $M_{\mu}=V \Sigma U^{T}$, and let $W$ be the left singular vectors (columns of $V$ ) corresponding to the $m$ largest singular values. Compute the matrix $M=$ $\left(W^{T} M_{\mu} W\right)\left(W^{T} M_{\lambda} W\right)^{-1}$.

2. Compute the eigenvector decomposition $M=P D P^{-1}$.

3. Output columns of $W P$.

The columns $C_{i}=W P_{i}$ are eigenvectors computed in subroutine Diagonalize. Ideally, we would like these to equal $A_{i}^{\odot d / 2}$. We are going to have errors introduced because of sampling, but in addition, since we are working in the complex field we do not have control over the phase of $C_{i}$ (the output of Diagonalize obtained in Step 3 of Tensor Decomposition), and for $\rho \in \mathbb{C}$ with $|\rho|=1, \rho C_{i}$ is also a valid output of Diagonalize. In Step 3 of Tensor Decomposition, we recover the correct phase of $C_{i} \in \mathbb{C}^{n}$ which will give us a vector in $C_{i}^{\prime} \in \mathbb{R}^{n}$. We do this by choosing the phase maximizing the norm of the real part.

In Step 4, we have $v^{\otimes d}+E$, where $E$ is an error tensor, and we want to recover $v$. We can do this approximately when $\|E\|_{F}$ is sufficiently small just by reading off a one-dimensional slice (e.g. a column in the case of matrices) of $v^{\otimes d}+E$ (say the one with the maximum norm). 


\section{Tensor Decomposition $\left(T_{\mu}, T_{\lambda}\right)$}

1. Flatten the tensors to square matrices to obtain $M_{\mu}=\tau^{-1}\left(T_{\mu}\right)$ and $M_{\lambda}=\tau^{-1}\left(T_{\lambda}\right)$.

2. $W P=$ Diagonalize $\left(M_{\mu}, M_{\lambda}\right)$.

3. For each column $C_{i}$ of $W P$, let $C_{i}^{\prime}:=\operatorname{Re}\left(e^{i \theta^{*}} C_{i}\right) /\left\|\operatorname{Re}\left(e^{i \theta^{*}} C_{i}\right)\right\|$ where $\theta^{*}=$ $\operatorname{argmax}_{\theta \in[0,2 \pi]}\left(\left\|\operatorname{Re}\left(e^{i \theta} C_{i}\right)\right\|\right)$.

4. For each column $C_{i}^{\prime}$, let $v_{i} \in \mathbb{R}^{n}$ be such that $v_{i}^{\otimes d / 2}$ is the best rank-1 approximation to $\tau\left(C_{i}^{\prime}\right)$.

For the computation of eigenvectors of diagonalizable (but not normal) matrices over the complex numbers, we can employ any of the several algorithms in the literature (see for example [36, 56] for a number of algorithms used in practice). In general, these algorithms employ the same atomic elements as the normal case (Jacobi iterations, Householder transformations etc.), but in more sophisticated ways. The perturbation analysis of these algorithms is substantially more involved than for normal matrices; in particular, it is not necessarily the case that a (small) perturbation to a diagonalizable matrix results in another diagonalizable matrix. We contend with all these issues in Section 5.3 In particular we note that while exact analysis is relatively straightforward (Theorem 5.3), a robust version that recovers the common decomposition of the given tensors takes considerable additional care (Theorem 5.4).

\subsection{Underdetermined ICA}

For underdetermined ICA we compute the higher derivative tensors of the second characteristic function $\psi_{x}(u)=\log \left(\phi_{x}(u)\right)$ at two random points and run the tensor decomposition algorithm from the previous section.

\section{Underdetermined $\operatorname{ICA}(\sigma)$}

1. (Compute derivative tensor) Pick independent random vectors $\alpha, \beta \sim$ $N\left(0, \sigma^{2} I_{n}\right)$. For even $d$ estimate the $d^{t h}$ derivative tensors of $\psi_{x}(u)$ at $\alpha$ and $\beta$ as $T_{\alpha}=D_{u}^{d} \psi_{x}(\alpha)$ and $T_{\beta}=D_{u}^{d} \psi_{x}(\beta)$.

2. (Tensor decomposition) Run Tensor Decomposition $\left(T_{\alpha}, T_{\beta}\right)$.

To estimate the $2 d^{\text {th }}$ derivative tensor of $\psi_{x}(u)$ empirically, one simply writes down the expression for the derivative tensor, and then estimates each entry from samples using the naive estimator.

The analysis roughly proceeds as follows: By (6) for tensors flattened into matrices we have $D_{u}^{2 d} \psi_{x}(\alpha)=$ $A^{\odot d} \operatorname{diag}\left(\partial_{t_{1}}^{2 d} \psi_{s}\left(A^{T} \alpha\right), \ldots, \partial_{t_{1}}^{2 d} \psi_{s}\left(A^{T} \alpha\right)\right)\left(A^{\odot d}\right)^{T}$ and $D_{u}^{2 d} \psi_{x}(\beta)=A^{\odot d} \operatorname{diag}\left(\partial_{t_{1}}^{2 d} \psi_{s}\left(A^{T} \beta\right), \ldots, \partial_{t_{1}}^{2 d} \psi_{s}\left(A^{T} \beta\right)\right)\left(A^{\odot d}\right)^{T}$.

Thus we have two tensors with shared rank-1 factors as in the tensor decomposition algorithm above. For our tensor decomposition to work, we require that all the ratios $\left(\partial_{t_{j}}^{2 d} \psi_{s}\left(A^{T} \alpha\right)\right) /\left(\partial_{t_{j}}^{2 d} \psi_{s}\left(A^{T} \beta\right)\right)$ for $j \in[m]$ be different from each other as otherwise the eigenspaces in the flattened forms will mix and we will not be able to uniquely recover the columns $A_{i}$. To this end, we will express $\partial_{t_{j}}^{2 d} \psi_{s}\left(A^{T} \alpha\right)$ as a low degree polynomial plus error term (which we will control by bounding the derivatives of $\psi_{s}$ ). The low degree polynomials will with high probability take on sufficiently different values for $A^{T} u$ and $A^{T} v$, which in turn guarantees that their ratios, even with the error terms, are quite different.

Our analysis for both parts might be of interest for other problems. On the way to doing this in full generality for underdetermined ICA, we first consider the special case of $d=2$, which will already involve several of these concepts and the algorithm itself is just PCA reweighted with Fourier weights. 


\section{Fully determined independent component analysis}

We begin with the case of standard or fully determined ICA where the transformation matrix $A \in \mathbb{R}^{n \times n}$ is full rank. With a slight loss of generality, we assume that $A$ is unitary. If $A$ is not unitary, we can simply make it approximately so by placing the entire sample in isotropic position. Rigorously arguing about this will require an additional error analysis; we will omit such details for the sake of clarity. In any case, our algorithm for underdetermined ICA does not (and cannot) make any such assumption. Our algorithm computes the eigenvectors of a covariance matrix reweighted according to random Fourier coefficients.

\section{Fourier $\mathbf{P C A}(\sigma)$}

1. (Isotropy) Get a sample $S$ from the input distribution and use them to find an isotropic transformation $B^{-1}$ with

$$
B^{2}=\frac{1}{|S|} \sum_{x \in S}(x-\bar{x})(x-\bar{x})^{T}
$$

2. (Fourier weights) Pick a random vector $u$ from $N\left(0, \sigma^{2} I_{n}\right)$. For every $x$ in a new sample $S$, compute $y=B^{-1} x$, and its Fourier weight

$$
w(y)=\frac{e^{i u^{T} y}}{\sum_{y \in S} e^{i u^{T} y}} .
$$

3. (Reweighted Covariance) Compute the covariance matrix of the points $y$ reweighted by $w(y)$

$$
\mu_{u}=\frac{1}{|S|} \sum_{y \in S} w(y) y \quad \text { and } \quad \Sigma_{u}=\frac{1}{|S|} \sum_{y \in S} w(y)\left(y-\mu_{u}\right)\left(y-\mu_{u}\right)^{T} .
$$

4. Compute the eigenmatrix $V$ of $\Sigma_{u}$ and output $B V$.

Formally, this algorithm is subsumed by our work on underdetermined ICA in Section 6 , but both the algorithm and its analysis are substantially simpler than the general case, but we retain the essential elements of our technique - fourier transforms, polynomial anti-concentration and derivative truncation. On the other hand, it does not require the machinery of our tensor decomposition in Section 5.

We make the following comments regarding the efficient realisation of this algorithm. The matrix $\Sigma_{u}$ in the algorithm is complex and symmetric, and thus is not Hermitian; its eigenvalue decomposition is more complicated than the usual Hermitian/real-symmetric case. It can be computed in one of two ways. One is to compute the SVD of $\Sigma_{u}$ (i.e., compute the eigenvalue decomposition of $\Sigma_{u} \Sigma_{u}^{*}$ which is a real symmetric matrix). Alternatively, we can exploit the fact that the real and complex parts have the same eigenvectors, and hence by carefully examining the real and imaginary components, we can recover the eigenvectors. We separate $\Sigma_{u}=\operatorname{Re}\left(\Sigma_{u}\right)+i \operatorname{Im}\left(\Sigma_{u}\right)$ into its real part and imaginary part, and use an SVD on Re $\left(\Sigma_{u}\right)$ to partition its eigenspace into subspaces with close eigenvalues, and then an SVD of $\operatorname{Im}\left(\Sigma_{u}\right)$ in each of these subspaces. Both methods need some care to ensure that eigenvalue gaps in the original matrix are preserved, an important aspect of our applications. We complete the algorithm description for ICA by giving a precise method for determining the eigenmatrix $V$ of the reweighted sample covariance matrix $\Sigma_{u}$. This subroutine below translates a gap in the complex eigenvalues of $\Sigma_{u}$ into observable gaps in the real part.

1. Write $\Sigma_{u}=\operatorname{Re}\left(\Sigma_{u}\right)+i \operatorname{Im}\left(\Sigma_{u}\right)$. Note that both the component matrices are real and symmetric.

2. Compute the eigendecomposition of $\operatorname{Re}\left(\Sigma_{u}\right)=U \operatorname{diag}\left(r_{i}\right) U^{T}$. 
3. Partition $r_{1}, \ldots, r_{n}$ into blocks $R_{1}, \ldots, R_{l}$ so that each block contains a subsequence of eigenvalues and the gap between consecutive blocks is at least $\epsilon_{0}$, i.e., $\min _{r \in R_{j}, s \in R_{j+1}} r-s \geq \epsilon_{0}$. Let $U_{j}$ be the eigenvectors corresponding to block $R_{j}$.

4. For each $1 \leq j \leq l$, compute the eigenvectors of $U_{j}^{T} \operatorname{Im}\left(\Sigma_{u}\right) U_{j}$ and output $V$ as the full set of eigenvectors (their union).

Lemma 4.1. Suppose $\Sigma_{u}$ has eigenvalues $\lambda_{1}, \ldots, \lambda_{n}$ and $\epsilon=\min _{i \neq j} \min \left\{\operatorname{Re}\left(\lambda_{i}\right)-\operatorname{Re}\left(\lambda_{j}\right), \operatorname{Im}\left(\lambda_{i}\right)-\right.$ $\left.\operatorname{Im}\left(\lambda_{j}\right)\right\}$. Then, with $\epsilon_{0}=\epsilon / n$, the above algorithm will recover the eigenvectors of $\Sigma_{u}$.

Proof. The decomposition of the matrix $\operatorname{Re}\left(\Sigma_{u}\right)$, will accurately recover the eigensubspaces for each block (since their eigenvalues are separated). Moreover, for each block $U_{j} \operatorname{diag}\left(r_{i}\right) U_{j}^{T}$, the real eigenvalues $r_{i}$ are within a range less than $\epsilon$ (since each consecutive pair is within $r_{i}-r_{i+1}<\epsilon / n$ ). Thus, for each pair $i, i+1$ in this block, we must have a separation of at least $\epsilon$ in the imaginary parts of $\lambda_{i}, \lambda_{i+1}$, by the definition of $\epsilon$. Therefore the eigenvalues of $Q_{j}=U_{j}^{T} \operatorname{Im}\left(\Sigma_{u}\right) U_{j}$ are separated by at least $\epsilon$ and we will recover the original eigenvectors accurately.

To perform ICA, we simply apply Fourier PCA to samples from the input distribution. We will show that for a suitable choice of $\sigma$ and sample size, this will recover the independent components to any desired accuracy. The main challenge in the analysis is showing that the reweighted covariance matrix will have all its eigenvalues spaced apart sufficiently (in the complex plane). This eigenvalue spacing depends on how far the component distributions are from being Gaussian, as measured by cumulants. Any non-Gaussian distribution will have a nonzero cumulant, and in that sense this is a complete method. We will quantify the gaps in terms of the cumulants to get an effective bound on the eigenvalue spacings. The number of samples is chosen to ensure that the gaps remain almost the same, and we can apply eigenvector perturbation theorems Davis-Kahan or Wedin to recover the eigenvectors to the desired accuracy.

\subsection{Overview of analysis}

Our main theorem in the analysis of this algorithm is as follows:

Theorem 4.2. Let $x \in \mathbb{R}^{n}$ be given by an ICA model $x=$ As where $A \in \mathbb{R}^{n \times n}$ is unitary and the $s_{i}$ are independent, $\mathbb{E}\left(s_{i}^{4}\right) \leq M_{4}$ for some constant, and for each $s_{i}$ there exists a $k_{i} \leq k$ such that $\left|\operatorname{cum}_{k_{i}}\left(s_{i}\right)\right| \geq \Delta$ (one of the first $k$ cumulants is large) and $\mathbb{E}\left(\left|s_{i}\right|^{k}\right) \leq M_{k}$. For any $\epsilon>0$, with the following setting of $\sigma$,

$$
\sigma=\frac{\Delta}{2 k !}\left(\frac{\sqrt{2 \pi}}{4(k-1) n^{2}}\right)^{k} \cdot \frac{1}{(2 e)^{k+1} M_{k} \log (4 n)^{k+1}},
$$

Fourier $\boldsymbol{P C A}$ will recover vectors $\left\{b_{1}, \ldots, b_{n}\right\}$ such that there exists signs $a_{i}= \pm 1$ satisfying

$$
\left\|A_{i}-b_{i}\right\| \leq \epsilon
$$

with high probability, using $(c k n)^{2 k^{2}+2}\left(M_{k} / \Delta\right)^{2 k+2} M_{4}^{2} / \epsilon^{2}$ samples.

Our analysis proceeds via the analysis of the Fourier transform: for a random vector $x \in \mathbb{R}^{n}$ distributed according to $f$, the characteristic function is given by the Fourier transform

$$
\phi(u)=\mathbb{E}\left(e^{i u^{T} x}\right)=\int f(x) e^{i u^{T} x} d x
$$

We favour the Fourier transform or characteristic function over the Laplace transform (or moment generating function) for the simple reason that the Fourier transform always exists, even for very heavy tailed distributions. In particular, the trivial bound $\left|e^{i t x}\right|=1$ means that once we have a moment bound, we can control the Fourier transform uniformly. 
We will actually employ the second characteristic function or cumulant generating function given by $\psi(u)=\log (\phi(u))$. Note that both these definitions are with respect to observed random vector $x$ : when $x$ arises from an ICA model $x=A s$, we will also define the component-wise characteristic functions with respect to the underlying $s_{i}$ variables $\phi_{i}\left(u_{i}\right)=\mathbb{E}\left(e^{i u_{i} s_{i}}\right)$ and $\psi_{i}\left(u_{i}\right)=\log \left(\phi_{i}\left(u_{i}\right)\right)$. Note that both these functions are with respect to the underlying random variables $s_{i}$ and not the observed random variables $x_{i}$. For convenience, we shall also write $g_{i}=\psi_{i}^{\prime \prime}$.

Note that the reweighted covariance matrix in our algorithm is precisely the Hessian second derivative matrix $D^{2} \psi$ :

$$
\Sigma_{u}=D^{2} \psi=\frac{\mathbb{E}\left(\left(x-\mu_{u}\right)\left(x-\mu_{u}\right)^{T} e^{i u^{T} x}\right)}{\mathbb{E}\left(e^{i u^{T} x}\right)},
$$

where $\mu_{u}=\mathbb{E}\left(x e^{i u^{T} x}\right) / \mathbb{E}\left(e^{i u^{T} x}\right)$. This matrix $D^{2} \psi$ has a very special form; suppose that $A=I_{n}$ :

$$
\psi(u)=\log \left(\mathbb{E}\left(e^{i u^{T} s}\right)\right)=\log \left(\mathbb{E}\left(\prod_{i=1}^{n} e^{i u_{i} s_{i}}\right)\right)=\sum_{i=1}^{n} \log \left(\mathbb{E}\left(e^{i u_{i} s_{i}}\right)\right)=\sum_{i=1}^{n} \psi_{i}\left(u_{i}\right) .
$$

Taking a derivative will leave only a single term

$$
\frac{\partial \psi}{\partial u_{i}}=\psi_{i}^{\prime}\left(u_{i}\right)
$$

And taking a second derivative will leave only the diagonal terms

$$
D^{2} \psi=\operatorname{diag}\left(\psi_{i}^{\prime \prime}\left(u_{i}\right)\right)=\operatorname{diag}\left(g_{i}\left(u_{i}\right)\right) .
$$

Thus, diagonalizing this matrix will give us the columns of $A=I_{n}$, provided that the eigenvalues of $D^{2} \psi$ are non-degenerate. In the general case when $A \neq I_{n}$, we can first place $x$ in isotropic position whence $A$ will be unitary. We perform the change of basis carefully in Section 4.2, obtaining that $D^{2} \psi=$ $A \operatorname{diag}\left(\psi_{i}^{\prime \prime}\left(\left(A^{T} u\right)_{i}\right)\right) A^{T}$. Now $D^{2} \psi$ is symmetric, but not Hermitian, and its eigenvalues are complex, but nonetheless a diagonalization suffices to give the columns of $A$.

To obtain a robust algorithm, we rely on the eigenvalues of $D^{2} \psi$ being adequately spaced (so that the error arising from sampling does not mix the eigenspaces, hence columns of $A$ ). Thus, we inject some randomness by picking a random Fourier coefficient, and hope that the $g_{i}\left(u_{i}\right)$ are sufficiently anti-concentrated. To this end, we will truncate the Taylor series of $g_{i}$ to $k^{t h}$ order, where the $k^{\text {th }}$ cumulant is one that differs from a gaussian substantially. The resulting degree $k$ polynomial will give us the spacings of the eigenvalues via polynomial anti-concentration estimates in Section 4.3, and we will control the remaining terms from order $k+1$ and higher by derivative estimates in Section 4.4. Notably, the further that $s_{i}$ is from being a gaussian (in cumulant terms), the stronger anti-concentration. We will pick the random Fourier coefficient $u$ according to a Gaussian $N\left(0, \sigma^{2} I_{n}\right)$ and we will show that with high probability for all pairs $i, j$ we have

$$
\left|g_{i}\left(\left(A^{T} u\right)_{i}\right)-g_{j}\left(\left(A^{T} u\right)_{j}\right)\right| \geq \delta .
$$

Critical to our analysis is the fact that $\left(A^{T} u\right)_{i}$ and $\left(A^{T} u\right)_{j}$ are both independent Gaussians since the columns of $A$ are independent by our assumption of isotropic position (Section 4.5). We then go onto compute the sample complexity required to maintain these gaps in Section 4.6 and conclude with the proof of correctness for our algorithm in Section 4.7.

In case more than one of the variables are (standard) Gaussians, then a quick calculation will verify that $\psi_{i}^{\prime \prime}\left(u_{i}\right)=1$. Thus, in the presence of such variables the eigenvectors corresponding to the eigenvalue 1 are degenerate and we can not resolve between any linear combination of such vectors. Thus, the model is indeterminate when some of the underlying random variables are too gaussian. To deal with this, one typically hypothesizes that the underlying variables $s_{i}$ are different from Gaussians. One commonly used way is to postulate that for each $s_{i}$ the fourth moment or cumulant differs from that of a Gaussian. We weaken this assumption, and only require that some moment is different from a Gaussian.

The Gaussian function plays an important role in harmonic analysis as the eigenfunction of the Fourier transform operator, and we exploit this property to deal with additive Gaussian noise in our model in Section 4.8 . 


\subsection{Eigenvalues}

As noted previously, when $A=I_{n}$, we have $D^{2} \psi=\operatorname{diag}\left(\psi_{i}^{\prime \prime}\left(u_{i}\right)\right)$. When $A \neq I_{n}$ we have

Lemma 4.3. Let $x \in \mathbb{R}^{n}$ be given by an ICA model $x=$ As where $A \in \mathbb{R}^{n \times n}$ is a unitary matrix and $s \in \mathbb{R}^{n}$ is an independent random vector. Then

$$
D^{2} \psi=A \operatorname{diag}\left(\psi_{i}^{\prime \prime}\left(\left(A^{T} u\right)_{i}\right)\right) A^{T} .
$$

This lemma is standard chain rule for multivariate functions. A more general version applying to higher derivative tensors is proved later in Section 6

\subsection{Anti-concentration for polynomials}

The main result of this section is an anti-concentration inequality for univariate polynomials under a Gaussian measure. While this inequality appears very similar to the inequality of Carbery-Wright [20] (cf. [52], Corollary 3.23), we are not able to derive our inequlity from it. The hypothesis of our inequality is weaker in that it only requires the polynomial to be monic instead of requiring the polynomial to have unit variance as required by Carbery-Wright; on the other hand it applies only to univariate polynomials.

Theorem 4.4 (Anti-concentration of a polynomial in Gaussian space). Let $p(x)$ be a degree $d$ monic polynomial over $\mathbb{R}$. Let $x \sim N\left(0, \sigma^{2}\right)$, then for any $t \in \mathbb{R}$ we have

$$
\operatorname{Pr}(|p(x)-t| \leq \epsilon) \leq \frac{4 d \epsilon^{1 / d}}{\sigma \sqrt{2 \pi}}
$$

For most of the proof we will work with the Lebesgue measure; the proof for the Gaussian measure will follow immediately. Our starting point is the following lemma which can be derived from the properties of Chebyshev polynomials ([17, Sec 2.1, Exercise 7); we include a proof for completeness. For interval $[a, b]$, define the supremum norm on real-valued functions $f$ defined on $[a, b]$ by

$$
\|f(x)\|_{[a, b]}:=\left\|f(x) \chi_{[a, b]}\right\|_{\infty}=\sup _{x \in[a, b]}|f(x)| .
$$

Then we have

Lemma 4.5. The unique degree $d$ monic polynomial minimising $\|p(x)\|_{[a, b]}$ is given by

$$
p(x)=2\left(\frac{b-a}{4}\right)^{d} T_{d}\left(\frac{2 x-a-b}{b-a}\right),
$$

where $T_{d}$ is the $d^{\text {th }}$ Chebyshev polynomial.

Proof. We already know (see [17]) that for the interval $[-1,1]$ the unique monic polynomial of degree $d$ which minimizes $\|p(x)\|_{[-1,1]}$ is given by $2^{1-d} T_{d}(x)$. Map the interval $[a, b]$ to $[-1,1]$ using the affine map $f(x)=$ $(2 x-a-b) /(b-a)$ which satisfies $f(a)=-1$ and $f(b)=1$. Then $((b-a) / 2)^{d} 2^{1-d} T_{d}(x)=2((b-a) / 4)^{d} T_{d}(x)$ is the unique monic polynomial minimizing $\|\cdot\|_{[a, b]}$. For if it were not, we could use such a polynomial to construct another monic polynomial (by reversing the above transformation) which contradicts the fact that Chebyshev polynomials are the unique monic minimizers of $\|\cdot\|_{[-1,1]}$.

From this we have the following lemma.

Lemma 4.6. Let $p(x)$ be a degree $d$ monic polynomial over $\mathbb{R}$. Fix $\epsilon>0$, then for every $x$, there exists an $x^{\prime}$ where $\left|x-x^{\prime}\right| \leq \epsilon$ and $\left|p(x)-p\left(x^{\prime}\right)\right| \geq 2(\epsilon / 2)^{d}$. 
Proof. We will translate the polynomial $p$ to obtain the polynomial $q(y)$ as follows:

$$
q(y)=p(y+x)-p(x) .
$$

Observe that $q(y)$ is a monic polynomial and $q(0)=0$. Now suppose that for all points $x^{\prime} \in[x-\epsilon, x+\epsilon]$, we have $\left|p(x)-p\left(x^{\prime}\right)\right|<(\epsilon / 2)^{d}$, then for all $y \in[-\epsilon, \epsilon]$, we must have $|q(y)|<2(\epsilon / 2)^{d}$.

But, from the previous lemma, we know that for the interval $[-\epsilon, \epsilon]$, the minimum $L^{\infty}$-norm on the interval for any monic polynomial is attained by $r(y)=2(\epsilon / 2)^{d} T_{d}(y / \epsilon)$. The value of this minimum is $2(\epsilon / 2)^{d}$.

We can use the above lemma to given an upper bound on the measure of the set where a polynomial stays within a constant sized band:

Lemma 4.7. Let $p(x)$ be a degree $d$ monic polynomial. Then for any interval $[a, b]$ where $b-a=\epsilon$ we have

$$
\mu(x \in \mathbb{R}: p(x) \in[a, b]) \leq 4 d\left(\frac{\epsilon}{2}\right)^{1 / d},
$$

where $\mu$ denotes the usual Lebesgue measure over $\mathbb{R}$.

Proof. Since $p$ is a continuous function so, $p^{-1}([a, b])=\cup_{i} I_{i}$ where $I_{i}$ are disjoint closed intervals. There are at most $d$ such intervals: every time $p(x)$ exits and re-enters the interval $[a, b]$ there must be a change of sign in the derivative $p^{\prime}(x)$ at some point in between. Since $p^{\prime}(x)$ is a degree $d-1$ polynomial, there can only be $d-1$ changes of sign.

Next, suppose that $\left|I_{i}\right|>4(\epsilon / 2)^{1 / d}$, then there exists an interval $\left[x-2\left(\epsilon^{\prime} / 2\right)^{1 / d}, x+2\left(\epsilon^{\prime} / 2\right)^{1 / d}\right] \subseteq I_{i}$, where $\epsilon^{\prime}>\epsilon$. Then, by applying Lemma 4.6. there exists a point $x^{\prime}$ such that $\left|x-x^{\prime}\right| \leq 2\left(\epsilon^{\prime} / 2\right)^{1 / d}$ but

$$
\begin{aligned}
\left|p(x)-p\left(x^{\prime}\right)\right| & \geq 2\left[\frac{1}{2} \cdot 2\left(\frac{\epsilon^{\prime}}{2}\right)^{1 / d}\right]^{d} \\
& \geq \epsilon^{\prime}>\epsilon .
\end{aligned}
$$

This implies that $x^{\prime} \notin[a, b]$, which is a contradiction. Hence we must have $\left|I_{i}\right| \leq 4(\epsilon / 2)^{1 / d}$ and

$$
\sum_{i}\left|I_{i}\right| \leq d \max _{i}\left|I_{i}\right| \leq 4 d(\epsilon / 2)^{1 / d}
$$

as required.

We can now give the proof for Theorem 4.4 :

Proof of Theorem 4.4. We know that the Lebesgue measure of the set for which $p(x) \in[t-\epsilon, t+\epsilon]$ is given by Lemma 4.7. Then multiplying by the maximum density of a Gaussian $1 / \sigma \sqrt{2 \pi}$ gives us the desired bound.

\subsection{Truncation Error}

Let us expand out the function $g_{i}=\psi_{i}^{\prime \prime}$ as a Taylor series with error estimate:

Theorem 4.8 (Taylor's theorem with remainder). Let $f: \mathbb{R} \rightarrow \mathbb{R}$ be a $C^{n}$ continuous function over some interval I. Let $a, b \in I$, then

$$
f(b)=\sum_{k=1}^{n-1} \frac{f^{(k)}(a)}{k !}(b-a)^{k}+\frac{f^{(n)}(\xi)}{n !}(b-a)^{n},
$$

for some $\xi \in[a, b]$. 
To this end, we write

$$
g_{i}\left(u_{i}\right)=p_{i}\left(u_{i}\right)+\frac{g^{(k)}(\xi)}{k !} u_{i}^{k}
$$

where $\xi \in\left[0, u_{i}\right]$ and $p_{i}$ is a polynomial of degree $(k-1)$.

To bound the error term in (9), we observe that it suffices to bound $\left[\log \left(\phi_{i}\right)\right]^{(k)}\left(u_{i}\right)$ using the following lemma.

Lemma 4.9. Let $x \in \mathbb{R}$ be a random variable with finite $k$ absolute moments, and let $\phi(u)$ be the associated characteristic function. Then

$$
\left|[\log (\phi)]^{(k)}(u)\right| \leq \frac{2^{k-1}(k-1) ! \mathbb{E}\left(|x|^{k}\right)}{|\phi(u)|^{k}}
$$

Proof. We will compute the derivatives of $\psi(u)=\log \phi(u)$ as follows: we proceed recursively with $\psi^{\prime}(u)=$ $\phi^{\prime}(u) / \phi(u)$ as our base case. Let $\psi^{(d)}$ be given by the ratio of two functions, a numerator function $N(u ; d)$ and a denominator function $D(u ; d)$, with no common factors and $N(u ; d)$ is the sum of terms of the form $\prod_{j=1}^{d} \phi^{\left(i_{j}\right)}(u)$ where the coefficient of each term is \pm 1 . Some useful properties of functions $N(u ; d), D(u ; d)$ are summarized in the following claim.

Claim 4.10. For $d \geq 1$, functions $N(u ; d)$ and $D(u ; d)$ satisfy

1. $D(u ; d)=\phi(u)^{d}$.

2. For each term of $N(u ; d), \sum_{j=1}^{d} i_{j} \leq d$.

3. For each term of $N(u ; d)$, the total number of factors of $\phi$ and its derivatives is at most $d$.

4. For $d \geq 1$, there are at most $2^{d-1}(d-1)$ ! terms in $N(u ; d)$.

Proof. We will prove all these via induction over $d$. Clearly these are all true for the base case $d=1$. Assume that all four facts are true for some $d$, we will now examine the case for $d+1$.

Writing $\psi^{(d+1)}(u)$ as the derivative of $\psi^{(d)}(u)=N(u ; d) / D(u ; d)$ and canceling common factors gives

$$
\begin{aligned}
\psi^{(d+1)}(u)= & \frac{N^{\prime}(u ; d) D(u ; d)-N(u ; d) D^{\prime}(u ; d)}{D(u ; d)^{2}} \\
& =\frac{N^{\prime}(u ; d) \phi(u)^{d}-N(u ; d) d \phi(u)^{d-1} \phi^{\prime}(u)}{\phi(u)^{2 d}} \\
& =\frac{N^{\prime}(u ; d) \phi(u)-d \phi^{\prime}(u) N(u ; d)}{\phi(u)^{d+1}} .
\end{aligned}
$$

Observing that there is always a term in $N(u ; d)=\phi^{\prime}(u)^{d}$, we can not cancel any further factors of $\phi(u)$. Hence $D(u ; d)=\phi(u)^{d}$, proving the first part of the claim.

The second and third parts of the claim follow immediately from the final expression for $\psi^{(d+1)}(u)$ above and our inductive hypothesis.

To prove the fourth part, let $T(d)$ denote the total number of terms in $N(u ; d)$, then by part 3 and the expansion in (10), we have $T(d+1) \leq d T(d)+d T(d) \leq 2 d T(d)$. From this $T(d+1) \leq 2^{d} d$ ! follows immediately.

Returning to the proof of Lemma 4.9, for $d \leq k$ we observe that

$$
\left|\phi^{(d)}(u)\right|=\left|\mathbb{E}\left((i x)^{d} e^{i u^{T} x}\right)\right| \leq \mathbb{E}\left(\left|(i x)^{d} e^{i u^{T} x}\right|\right) \leq \mathbb{E}\left(|x|^{d}\right) .
$$


Thus, for each term of $N(u ; d)$ :

$$
\left|\prod_{j=1}^{d} \phi^{\left(i_{j}\right)}(u)\right| \leq \prod_{j=1}^{d}\left|\phi^{\left(i_{j}\right)}(u)\right| \leq \prod_{j=1}^{d} \mathbb{E}\left(|x|^{i_{j}}\right)^{\mathrm{Fac}} \mathbb{1 0 . 6} \mathbb{E}\left(|x|^{\sum_{j=1}^{d} i_{j}}\right) \leq \mathbb{E}\left(|x|^{d}\right) .
$$

Combining Claim 4.10 with the previous equation, and noticing that we never need to consider absolute moments of order higher than $k$ (which are guaranteed to exist by our hypothesis), gives the desired conclusion.

To conclude this section, we observe that if the distribution of $x \in \mathbb{R}$ is isotropic then for $u \in(-1,1)$ we have

$$
\phi_{x}(u)=\phi_{x}(0)+\phi_{x}^{\prime}(0) u+\frac{\phi_{x}^{\prime \prime}(\xi)}{2} u^{2}
$$

where $\xi \in[0, u]$. We have $\phi_{x}(0)=1, \phi_{x}^{\prime}(0)=\mathbb{E}(x)=0$ and $\left|\phi_{x}^{\prime \prime}(\xi)\right| \leq \mathbb{E}\left(|x|^{2}\right)=1$ by the isotropic position assumption. Thus, for $u \in[-1 / 4,1 / 4]$, Lemma 4.9 gives us

$$
\left|\left[\log \left(\phi_{x}\right)\right]^{(k)}(u)\right| \leq \mathbb{E}\left(|x|^{k}\right) k^{k}
$$

\subsection{Eigenvalue spacings}

We will apply Theorem 4.4 to the truncated Taylor polynomials $p_{i}$ of the functions $\psi_{i}^{\prime \prime}$, and bound the truncation error to be at most a constant fraction of the desired anti-concentration.

Theorem 4.11. Let $s \in \mathbb{R}^{n}$ be a random vector with indpendent components. For $t \in \mathbb{R}^{n}$, let $\psi(t)=$ $\log \mathbb{E}\left(e^{i t^{T} s}\right)$ be the second characteristic function of s. Suppose we are given the following data and conditions:

1. Integer $k>2$ such that $\mathbb{E}\left(\left|s_{i}\right|^{k}\right)$ exists for all $i \in[n]$.

2. $\Delta>0$ such that for each $i \in[n]$, there exists $2<k_{i}<k$ such that $\left|\operatorname{cum}_{k_{i}}\left(s_{i}\right)\right| \geq \Delta$.

3. $M_{2}>0$ such that $\mathbb{E}\left(s_{i}^{2}\right) \leq M_{2}$ for $i \in[n]$.

4. $M_{k}>0$ such that $\mathbb{E}\left(\left|s_{i}\right|^{k}\right) \leq M_{k}$ for $i \in[n]$.

5. $g_{i}\left(t_{i}\right):=\frac{\partial^{2} \psi(t)}{\partial t_{i}^{2}}$.

6. $\tau \sim N\left(0, \sigma^{2} I_{n}\right)$ where $\sigma=\min \left(1, \frac{1}{2 \sqrt{2 M_{2} \log 1 / q}}, \sigma^{\prime}\right)$ and

$$
\sigma^{\prime}=\left(\frac{3}{8}\right)^{k+1} \cdot \frac{k-1}{k !} \cdot\left(\frac{\sqrt{2 \pi}}{4 k}\right)^{k-2} \cdot \frac{q^{k-2}}{(\sqrt{2 \log (1 / q)})^{k-1}} \cdot \frac{\Delta}{M_{k}},
$$

and $0<q \leq 1 / 3$.

Then with probability at least $1-n^{2} q$, for all distinct $i, j$ we have

$$
\left|g_{i}\left(\tau_{i}\right)-g_{j}\left(\tau_{j}\right)\right| \geq \frac{\Delta}{2(k-2) !}\left(\frac{\sqrt{2 \pi} \sigma q}{4 k}\right)^{k-2} .
$$


Proof. We will argue about the spacing $\left|g_{1}\left(\tau_{1}\right)-g_{2}\left(\tau_{2}\right)\right|$, and then use the union bound to get that none of the spacings is small with high probability. Since $s_{1}$ has first $k$ moments, we can apply Taylor's theorem with remainder (Actually one needs more care as that theorem was stated for functions of type $\mathbb{R} \rightarrow \mathbb{R}$, whereas our function here is of type $\mathbb{R} \rightarrow \mathbb{C}$. To this end, we can consider the real and imaginary parts of the function separately and apply Theorem 4.8 to each part; we omit the details.) Applying Theorem 4.8 gives

$$
g_{1}\left(t_{1}\right)=-\sum_{l=2}^{k_{1}} \operatorname{cum}_{l}\left(s_{1}\right) \frac{\left(i t_{1}\right)^{l-2}}{(l-2) !}+R_{1}\left(t_{1}\right) \frac{\left(i t_{1}\right)^{k_{1}-1}}{\left(k_{1}-1\right) !} .
$$

Truncating $g_{1}$ after the degree $\left(k_{1}-2\right)$ term yields a polynomial $p_{1}\left(t_{1}\right)$. Denote the truncation error by $\rho_{1}\left(t_{1}\right)$. Then, fixing $t_{2}$ arbitrarily and setting $z=g_{2}\left(t_{2}\right)$ for brevity, we have

$$
\begin{aligned}
\left|g_{1}\left(t_{1}\right)-g_{2}\left(t_{2}\right)\right| & =\left|p_{1}\left(t_{1}\right)+\rho_{1}\left(t_{1}\right)-z\right| \\
& \geq\left|p_{1}\left(t_{1}\right)-z\right|-\left|\rho_{1}\left(t_{1}\right)\right| .
\end{aligned}
$$

We will show that $\left|p_{1}\left(t_{1}\right)-z\right|$ is likely to be large and $\left|\rho_{1}\left(t_{1}\right)\right|$ is likely to be small. Noting that $\frac{\left(k_{1}-2\right) !}{i^{k_{1}-2} \operatorname{cum}_{k_{1}}\left(s_{1}\right)} p_{1}\left(t_{1}\right)$ is monic of degree $k_{1}-2$ (but with coefficients from $\mathbb{C}$ ), we apply our anti-concentration result in Theorem 4.4. Again, although that theorem was proven for polynomials with real coefficients, its application to the present situation is easily seen to go through without altering the bound by considering the real and imaginary parts separately. In the following, the probability is for $t_{1} \sim N\left(0, \sigma^{2}\right)$.

$$
\operatorname{Pr}\left(\left|p_{1}\left(t_{1}\right)-z\right| \leq \epsilon_{1}\right) \leq \frac{4\left(k_{1}-2\right)}{\sigma \sqrt{2 \pi}}\left(\frac{\epsilon_{1}\left(k_{1}-2\right) !}{\left|\operatorname{cum}_{k_{1}}\left(s_{1}\right)\right|}\right)^{1 /\left(k_{1}-2\right)} \leq \frac{4 k_{1}}{\sigma \sqrt{2 \pi}}\left(\frac{\epsilon_{1}\left(k_{1}-2\right) !}{\Delta}\right)^{1 /\left(k_{1}-2\right)} .
$$

Setting

$$
\epsilon_{1}:=\frac{\Delta}{\left(k_{1}-2\right) !}\left(\frac{\sqrt{2 \pi} \sigma q}{4 k_{1}}\right)^{\left(k_{1}-2\right)} \leq \frac{\Delta}{(k-2) !}\left(\frac{\sqrt{2 \pi} \sigma q}{4 k}\right)^{(k-2)}
$$

we have

$$
\operatorname{Pr}\left(\left|p_{1}\left(t_{1}\right)-z\right| \leq \epsilon\right) \leq q .
$$

Next we bound the truncation error and show that $\left|\rho_{1}\left(t_{1}\right)\right| \leq \epsilon / 2$ with probability at least $1-\frac{q}{\sqrt{\pi \log 1 / q}}$. Applying Lemma 4.9, the error introduced is

$$
\left|\rho_{1}\left(t_{1}\right)\right| \leq \frac{k_{1} ! 2^{k_{1}} \mathbb{E}\left(\left|s_{1}\right|^{k_{1}+1}\right)}{\left|\phi_{1}\left(t_{1}\right)\right|^{k_{1}+1}} \cdot \frac{t_{1}^{k_{1}-1}}{\left(k_{1}-1\right) !} .
$$

We now lower bound the probability that $\left|t_{1}\right|$ is small when $t_{1} \sim N\left(0, \sigma^{2}\right)$ :

$$
\operatorname{Pr}\left(\left|t_{1}\right| \leq \sigma \sqrt{2 \log 1 / q}\right) \geq 1-\frac{q}{\sqrt{\pi \log 1 / q}} .
$$

The computation above used Claim 10.3

Thus with probability at least $1-\frac{q}{\sqrt{\pi \log 1 / q}}$ we have

$$
\left|\rho_{1}\left(t_{1}\right)\right| \leq \frac{k_{1} ! 2^{k_{1}} M_{k}}{(3 / 4)^{k_{1}+1}\left(k_{1}-1\right) !} \cdot(\sigma \sqrt{2 \log 1 / q})^{k_{1}-1},
$$

here we used that by our choice of $\sigma$ we have $\sigma \sqrt{2 \log 1 / q} \leq \frac{1}{2 \sqrt{M_{2}}}$, hence Lemma10.1 gives that $\left|\phi\left(t_{1}\right)\right| \geq 3 / 4$. 
Now for $\left|t_{1}\right| \leq \sigma \sqrt{2 \log 1 / q}$ we want

$$
\left|\rho_{1}\left(t_{1}\right)\right| \leq \epsilon_{1} / 2 .
$$

This is seen to be true by plugging in the value of $\epsilon_{1}$ from (13) and the bound on $\rho_{1}\left(t_{1}\right)$ from (14) and our choice of $\sigma$.

Thus we have proven that $\left|g_{1}\left(t_{1}\right)-g_{2}\left(t_{2}\right)\right| \geq \epsilon / 2$ with probability at least $1-\left(q+\frac{q}{\sqrt{\pi \log 1 / q}}\right) \geq 1-2 q$ (using $q \in(0,1 / 3])$. Now applying the union bound over all pairs we get the required bound.

\subsection{Sample complexity}

In this section, we bound the sample complexity of the algorithm. First we will show how many samples are necessary to estimate accurately the desired Fourier transforms $\mathbb{E}\left(e^{i u^{T} x}\right), \mathbb{E}\left(x e^{i u^{T} x}\right)$ and $\mathbb{E}\left(x x^{T} e^{i u^{T} x}\right)$.

Lemma 4.12. Let $x \in \mathbb{R}^{n}$ be a random vector. Fix $\epsilon>0$ and a vector $t \in \mathbb{R}^{n}$. Let $x^{(j)}$ be i.i.d. samples drawn according to $x$ then

$$
\left|\frac{1}{m} \sum_{j=1}^{m} e^{i u^{T} x^{(j)}}-\mathbb{E}\left(e^{i u^{T} x}\right)\right| \leq \epsilon,
$$

with probability at least $1-4 e^{-m \epsilon^{2} / 2}$.

Proof. Note that the random variables $e^{i u^{T} x}$ are bounded in magnitude by 1 . We separate out the real and imaginary components of $e^{i u^{T} x}$ and separately apply the Chernoff inequality.

In the most general setting, all we can do is bound the variance of our sample covariance matrix, and this will give a polynomial bound on the sample complexity.

Lemma 4.13. Suppose that the random vector $x \in \mathbb{R}^{n}$ is drawn from an isotropic distribution $F$. Then

$$
\begin{aligned}
\operatorname{Var}\left(x_{j} e^{i u^{T} x}\right) & \leq 1 \text { for } 1 \leq j \leq n, \\
\operatorname{Var}\left(x_{j}^{2} e^{i u^{T} x}\right) & \leq \mathbb{E}\left(x_{j}^{4}\right), \\
\operatorname{Var}\left(x_{i} x_{j} e^{i u^{T} x}\right) & \leq 1 \text { for } i \neq j .
\end{aligned}
$$

Proof.

$$
\operatorname{Var}\left(x_{j} e^{i u^{T} x}\right)=\mathbb{E}\left(x_{j}^{2}\right)-\left|\mathbb{E}\left(x_{j} e^{i u^{T} x}\right)\right|^{2} \leq 1 .
$$

The other parts are similar, with the last inequality using isotropy.

We can combine these concentration results for the Fourier derivatives to obtain the final sample complexity bound. Recall from (11) that we have in the interval $u \in[-1 / 4,1 / 4]$

$$
|g(u)| \leq \mathbb{E}\left(|x|^{2}\right) k^{k} \leq 16
$$

We can now give the sample complexity of the algorithm.

Corollary 4.14. Let $x=A s$ be an ICA model where $A \in \mathbb{R}^{n \times n}$ is a unitary matrix. Suppose that the random vector $s \in \mathbb{R}^{n}$ is drawn from an isotropic distribution, and that for each $s_{i}$, we have $\mathbb{E}\left(s_{i}^{4}\right) \leq M$. Fix $\epsilon>0$ and a vector $u \in \mathbb{R}^{n}$ where $\|u\| \leq 1 / 4$. Let $\hat{\Sigma}_{u}$ be the matrix estimated from $m$ independent samples of $x^{i}=A s^{i}$, then

$$
\left\|\hat{\Sigma}_{u}-\Sigma_{u}\right\|_{F} \leq \epsilon
$$

with probability at least $1-1 / n$ for $m \geq \operatorname{poly}(n, M) / \epsilon^{2}$. 
Proof. Apply Chebyshev's inequality along with the variance bounds. Since the Frobenius norm is unitarily invariant, we can consider the error in the basis corresponding to $s$. In this basis:

$$
\begin{aligned}
& \left\|\mathbb{E}\left(e^{i u^{T} s}(s-\tilde{\mu})(s-\tilde{\mu})^{T}-\tilde{\Sigma}_{u}\right)\right\| \\
& \leq\left\|\mathbb{E}\left(s s^{T} e^{i u^{T} s}\right)-\sum_{i=1}^{m}\left(s^{i}\right)\left(s^{i}\right)^{T} e^{i u^{T} s^{i}}\right\|+2\left\|\mathbb{E}\left(s \tilde{\mu}^{T} e^{i u^{T} s}\right)-\sum_{i=1}^{m} x \hat{\tilde{\mu}}^{T} e^{i u^{T} s}\right\| \\
& \quad+\left\|\mathbb{E}\left(\tilde{\mu} \tilde{\mu}^{T}\right)-\hat{\tilde{\mu}} \hat{\tilde{\mu}}^{T}\right\|\left|\mathbb{E}\left(e^{i u^{T} s}\right)\right| \\
& \leq \epsilon
\end{aligned}
$$

where the last bound is derived by apportioning $\epsilon / 5$ error to each term. Finally, we conclude by noting that by our choice of $t$, we have $\left|\mathbb{E}\left(e^{i u^{T} x}\right)\right| \geq 29 / 32$, and the multiplicative error due to the scaling by $1 / \mathbb{E}\left(e^{i u^{T} x}\right)$ is lower order in comparison to $\epsilon$.

For more structured distributions, e.g., logconcave distributions, or more generally distributions with subexpoential tails, much sharper bounds are known on the sample complexity of covariance estimation, see e.g., [57, 67, 60, 1].

\subsection{Proof of Theorem 4.2}

In this section we give the proof of correctness for our algorithm for ICA.

Proof of Theorem 4.2. In the exact case, the diagonal entries are given by $g_{i}\left(\left(A^{T} u\right)_{i}\right)$. Since $A$ is orthonormal, for any pair $\left(A^{T} u\right)_{i}=A_{i}^{T} u$ and $\left(A^{T} u\right)_{j}=A_{j}^{T} u$ have orthogonal $A_{i}$ and $A_{j}$, hence the arguments of $g_{i}$ and $g_{j}$ are independent Gaussians and Theorem 4.11 gives us the eigenvalue spacings of $\Sigma_{u}$ to be used in Lemma 8.1

In particular, the spacings are at least $\xi=\frac{\Delta}{2 k !}\left(\frac{\sqrt{2 \pi} \sigma}{4(k-1)^{n^{2}}}\right)^{k}$. Thus, with desired accuracy $\epsilon$ in Lemma 8.1. then we require the sampling error (in operator norm, which we upper bound using Frobenius norm) to be $\|E\|_{F} \leq \epsilon \xi /(\xi+\epsilon)$. We can then substitute this directly into Corollary 4.14 which gives the sample complexity.

\subsection{Gaussian noise}

The Gaussian function has several nice properties with respect to the Fourier transform, and we can exploit these to cancel out independent Gaussian noise in the problems that we study. To deal with Gaussian noise, when the observed signal $x=A s+\eta$ where $\eta$ is from an unknown Gaussian $N\left(\mu_{\eta}, R_{\eta}\right)$ which is independent of $s$, we can use the following modified algorithm.

1. Pick two different random Gaussian vectors $u, v$.

2. Compute $\Sigma=\Sigma_{0}, \Sigma_{u}$ and $\Sigma_{v}$ as in the previous algorithm.

3. Output the eigenvectors of $\left(\Sigma_{u}-\Sigma\right)\left(\Sigma_{v}-\Sigma\right)^{-1}$.

Theorem 4.15. Let $x \in \mathbb{R}^{n}$ be given be a noisy independent components model $x=A s+\eta$, where $A \in \mathbb{R}^{n \times n}$ is a full rank matrix, and the noise vector $\eta$ has a Gaussian distribution. With sufficiently many samples, the modified algorithm outputs A. 
Proof. When $x=A s+\eta$, the function $\psi(u)=\log \left(\mathbb{E}\left(e^{i u^{T} x}\right)\right)$ can be written as

$$
\psi(u)=\log \left(\mathbb{E}\left(e^{i u^{T} x}\right)\right)+\log \left(\mathbb{E}\left(e^{i u^{T} \eta}\right)\right)
$$

Therefore,

$$
\begin{aligned}
D^{2} \psi_{u} & =A \operatorname{diag}\left(\psi_{i}^{\prime \prime}\left(A_{i}^{T} u\right)\right) A^{T}+\frac{\mathbb{E}\left(e^{i u^{T} \eta}\left(\eta-\mu_{\eta}\right)\left(\eta-\mu_{\eta}\right)^{T}\right)}{\mathbb{E}\left(e^{i u^{T} \eta}\right)} \\
& =A \operatorname{diag}\left(\psi_{i}^{\prime \prime}\left(A_{i}^{T} u\right)\right) A^{T}+\mathbb{E}\left(\left(\eta-\mu_{\eta}\right)\left(\eta-\mu_{\eta}\right)^{T}\right) \\
& =A \operatorname{diag}\left(\psi_{i}^{\prime \prime}\left(A_{i}^{T} u\right)\right) A^{T}+R_{\eta}
\end{aligned}
$$

where $\eta \sim N\left(\mu_{\eta}, R_{\eta}\right)$. Therefore,

$$
\Sigma_{u}-\Sigma=A\left(D_{u}-D\right) A^{T}
$$

with $D$ being the covariance matrix of $s$ and

$$
\left(\Sigma_{u}-\Sigma\right)\left(\Sigma_{v}-\Sigma\right)^{-1}=A\left(D_{u}-D\right)\left(D_{v}-D\right)^{-1} A^{-1} .
$$

The eigenvectors of the above matrix are the columns of $A$.

For a complete robustness analysis, one needs to control the spectral perturbations of the matrix $A\left(D_{u}-\right.$ $D)\left(D_{v}-D\right)^{-1} A^{-1}$ under sampling error. We omit this proof, but note that it follows easily using the techniques we develop for underdetermined ICA.

\section{$5 \quad$ Efficient tensor decompositions}

In this section we analyze the tensor decomposition algorithm, which will be our main tool for the underdetermined ICA problem.

\subsection{Algorithm}

Recall that our algorithm works by flattening tensors $T_{\mu}$ and $T_{\lambda}$ into matrices $M_{\mu}$ and $M_{\lambda}$ and then observing that the eigenvectors of $M_{\mu} M_{\lambda}^{-1}$ are vectors corresponding to flattened (vectorized) $A_{i}^{\otimes d / 2}$.

\section{$\operatorname{Diagonalize}\left(M_{\mu}, M_{\lambda}\right)$}

1. Compute the SVD of $M_{\mu}=V \Sigma U^{*}$, and let the $W$ be the left singular vectors (columns of $V$ ) corresponding to the $m$ largest singular values. Compute the matrix $M=$ $\left(W^{*} M_{\mu} W\right)\left(W^{*} M_{\lambda} W\right)^{-1}$.

2. Compute the eigenvector decomposition $M=P D P^{-1}$.

3. Output columns of $W P$.

In Step 3 of Tensor Decomposition, we get an approximation of $v_{i}^{\odot d / 2}$ up to a phase factor. We first correct the phase by maximizing the projection onto $\mathbb{R}^{n}$. To this end we prove

Lemma 5.1. Let $v \in \mathbb{C}^{n}$ and $u \in \mathbb{R}^{n}$ be unit vectors such that for some $\varphi \in[0,2 \pi]$ we have $\left\|e^{i \varphi} v-u\right\| \leq \epsilon$ for $0 \leq \epsilon \leq 1 / 2$. Let $\theta^{*}=\operatorname{argmax}_{\theta \in[0,2 \pi]}\left(\left\|\operatorname{Re}\left(e^{i \theta} v\right)\right\|\right)$ and $u^{\prime}=\operatorname{Re}\left(e^{i \theta^{*}} v\right) /\left\|\operatorname{Re}\left(e^{i \theta^{*}} v\right)\right\|$. Then there is $a$ sign $\alpha \in-1,1$ such that

$$
\left\|\alpha u-u^{\prime}\right\| \leq 11 \sqrt{\epsilon} .
$$




\section{Tensor Decomposition $\left(T_{\mu}, T_{\lambda}\right)$}

1. Flatten the tensors to obtain $M_{\mu}=\tau^{-1}\left(T_{\mu}\right)$ and $M_{\lambda}=\tau^{-1}\left(T_{\lambda}\right)$.

2. $W P=$ Diagonalize $\left(M_{\mu}, M_{\lambda}\right)$.

3. For each column $C_{i}$ of $W P$, let $C_{i}^{\prime}:=\operatorname{Re}\left(e^{i \theta^{*}} C_{i}\right) /\left\|\operatorname{Re}\left(e^{i \theta^{*}} C_{i}\right)\right\|$ where $\theta^{*}=$ $\operatorname{argmax}_{\theta \in[0,2 \pi]}\left(\left\|\operatorname{Re}\left(e^{i \theta} C_{i}\right)\right\|\right)$.

4. For each column $C_{i}^{\prime}$, let $v_{i} \in \mathbb{R}^{n}$ be such that $v_{i}^{\otimes d / 2}$ is the best rank-1 approximation to $\tau\left(C_{i}^{\prime}\right)$.

Proof. Without loss of generality, we will assume that $\varphi=0$, hence $\|v-u\| \leq \epsilon$. By the optimality of $\theta^{*}$

$$
\left\|\operatorname{Re}\left(e^{i \theta^{*}} v\right)\right\| \geq\|\operatorname{Re}(v)\| \geq 1-\epsilon
$$

Let us denote $v^{\prime}=e^{i \theta^{*}} v$, then we have $\left\|\operatorname{Re}\left(v^{\prime}\right)\right\|^{2}+\left\|\operatorname{Im}\left(v^{\prime}\right)\right\|^{2}=1$ which implies that $\left\|\operatorname{Im}\left(v^{\prime}\right)\right\|^{2} \leq 2 \epsilon-\epsilon^{2}<2 \epsilon$. Now using $\epsilon \leq 1 / 2$ we have

$$
\begin{aligned}
\left\|v^{\prime}-u^{\prime}\right\| & \leq\left\|\operatorname{Re}\left(v^{\prime}\right)-u^{\prime}\right\|+\left\|\operatorname{Im}\left(v^{\prime}\right)\right\| \\
& =\left\|\operatorname{Re}\left(v^{\prime}\right)-\frac{\operatorname{Re}\left(v^{\prime}\right)}{\left\|\operatorname{Re}\left(v^{\prime}\right)\right\|}\right\|+\left\|\operatorname{Im}\left(v^{\prime}\right)\right\| \\
& \leq\left\|\operatorname{Re}\left(v^{\prime}\right)\right\|\left(\frac{1}{1-\epsilon}-1\right)+\left\|\operatorname{Im}\left(v^{\prime}\right)\right\| \\
& \leq 2 \epsilon+\sqrt{2 \epsilon} \leq 4 \sqrt{\epsilon},
\end{aligned}
$$

and

$$
\left\|u^{\prime}-e^{i \theta^{*}} u\right\| \leq\left\|u^{\prime}-e^{i \theta^{*}} v\right\|+\left\|e^{i \theta^{*}} v-e^{i \theta^{*}} u\right\|=\left\|u^{\prime}-v^{\prime}\right\|+\|u-v\|<5 \sqrt{\epsilon} .
$$

This implies $\left|\operatorname{Re}\left(e^{i \theta^{*}}\right)\right| \geq 1-5 \sqrt{\epsilon}$. Hence there is a sign $\alpha \in-1,1$ such that $\left|e^{i \theta^{*}}-\alpha\right| \leq 10 \sqrt{\epsilon}$ (we omit some routine computations). Finally,

$$
\left\|u^{\prime}-\alpha u\right\| \leq\left\|u^{\prime}-e^{i \theta^{*}} u\right\|+\left\|e^{i \theta^{*}} u-\alpha u\right\| \leq 5 \sqrt{\epsilon}+10 \sqrt{\epsilon}=15 \sqrt{\epsilon}
$$

Lemma 5.2. For unit vector $v \in \mathbb{R}^{n}$ and a positive integer $d$, given $v^{\odot d}+E$, where $E$ is an error vector, we can recover $v^{\prime \prime}$ such that for some $\alpha \in\{-1,1\}$ we have

$$
\left\|v-\alpha v^{\prime \prime}\right\|_{2} \leq \frac{2\|E\|_{2}}{\beta-\|E\|_{2}}
$$

where $\beta=\frac{1}{n^{d / 2-1 / 2}}$.

Proof. Let's for a moment work with $v^{\odot d}$ (so there is no error), and then we will take the error into account. In this case we can essentially read $v$ off from $v^{\odot d}$. Each one-dimensional slice of $v^{\otimes d}$ (Note that as vectors, $v^{\odot d}$ and $v^{\otimes d}$ are the same; they differ only in how their entries are arranged: In the former, they are in a linear array and in the latter they are in an $n \times n \times \ldots \times n$ array. We will use them interchangeably, and we will also talk about $v^{\otimes d}+E$ which has the obvious meaning.) is a scaled copy of $v$. Let us choose the copy with the maximum norm. Since $\|v\|=1$, there is a coordinate $v(i)$ such that $|v(i)| \geq 1 / \sqrt{n}$. Thus 
there is a one-dimensional slice of $v^{\otimes d}$ with norm at least $\frac{1}{n^{d / 2-1 / 2}}=\beta$. Scaling this slice to norm 1 would result in $\alpha v$ for some $\alpha \in\{-1,1\}$. Now, when we do have error and get $v^{\otimes d}+E$, then we must have a one-dimensional slice $v^{\prime}$ of $v^{\otimes d}+E$ with norm at least $\beta-\|E\|_{2}$. Then after normalizing $v^{\prime}$ to $v^{\prime \prime}$, one can check that $\left\|\alpha v^{\prime \prime}-v\right\| \leq \frac{2\|E\|_{2}}{\beta-\|E\|_{2}}$ for some $\alpha \in\{-1,1\}$.

\subsection{Exact analysis}

We begin with the proof of the tensor decomposition theorem with access to exact tensors as stated in Theorem 1.2, This is essentially a structural results that says we can recover the rank-1 components when the ratios $\mu_{i} / \lambda_{i}$ are unique.

We first note that for a tensor $T_{\mu}$ with a rank-1 decomposition as in (10), that the flattened matrix version $M_{\mu}=\tau^{-1}\left(T_{\mu}\right)$ can be written as

$$
M_{\mu}=\left(A^{\odot d / 2}\right) \operatorname{diag}\left(\mu_{i}\right)\left(A^{\odot d / 2}\right)^{T} .
$$

We will argue that the diagonalisation step works correctly (we will write $B=A^{\odot d / 2}$ in what follows). The recovery of $A_{i}$ from the columns of $B$ follows by Lemma 5.1 above.

Our theorem is as follows (note that the first condition below is simply a normalisation of the eigenvectors):

Theorem 5.3. Let $M_{\mu}, M_{\lambda} \in \mathbb{C}^{p \times p}$ such that:

$$
M_{\mu}=B \operatorname{diag}\left(\mu_{i}\right) B^{T}, \quad \text { and } \quad M_{\lambda}=B \operatorname{diag}\left(\lambda_{i}\right) B^{T},
$$

where $B \in \mathbb{R}^{p \times m}$ and $\mu, \lambda \in \mathbb{C}^{m}$ for some $m \leq p$. Suppose that the following hold:

1. For each column $B_{i} \in \mathbb{R}^{m}$ of $B,\left\|B_{i}\right\|_{2}=1$,

2. $\sigma_{m}(B)>0$, and

3. $\mu_{i}, \lambda_{i} \neq 0$ for all $i$, and $\left|\frac{\mu_{i}}{\lambda_{i}}-\frac{\mu_{j}}{\lambda_{j}}\right|>0$ for all $i \neq j$.

Then Diagonalize $\left(M_{\mu}, M_{\lambda}\right)$ outputs the columns of $B$ up to sign and permutation.

Proof. By our assumptions, the image of $M_{\lambda}$ has dimension $m$ and the matrix $W \operatorname{computed}$ in Diagonalize $\left(M_{\mu}, M_{\lambda}\right)$ satisfies colspan $(W)=$ colspan $(B)$. Moreover, we could choose $W$ to have all entries real because $B$ is a real matrix; this will give that the ambiguities in the recovery of $B$ are in signs and not in phase. Since the columns of $W$ are orthonormal, the columns of $P:=W^{T} B$ all have unit norm and it is a full rank $m \times m$ matrix. So we can write

$$
\begin{aligned}
W^{T} M_{\mu} W & =P \operatorname{diag}\left(\mu_{i}\right) P^{T}, \\
\left(W^{T} M_{\lambda} W\right)^{-1} & =\left(P^{T}\right)^{-1} \operatorname{diag}\left(\lambda_{i}^{-1}\right) P^{-1} .
\end{aligned}
$$

Which gives

$$
\left(W^{T} M_{\mu} W\right)\left(W^{T} M_{\lambda} W\right)^{-1}=P \operatorname{diag}\left(\mu_{i} / \lambda_{i}\right) P^{-1} .
$$

Thus the colums of $P$ are the eigenvectors of $\left(W^{T} M_{\mu} W\right)\left(W^{T} M_{\lambda} W\right)^{-1}$, and thus our algorithm is able to recover the columns of $P$ up to sign and permutation. Let's call the matrix so recovered $P^{\prime}$. Denote by $P_{1}, \ldots, P_{m}$ the columns of $P$, and similarly for $P^{\prime}$ and $B$. Then $P^{\prime}$ is given by $P_{\pi(j)}^{\prime}=\alpha_{j} P_{j}$ where $\pi:[m] \rightarrow[m]$ is a permutation and $\alpha_{j} \in\{-1,+1\}$.

We now claim that $W P=W W^{T} B=B$. To see this, let $\hat{W}=\left[W, W^{\prime}\right]$ be an orthonormal basis that completes $W$. Then $\hat{W}^{T} \hat{W}=\hat{W} \hat{W}^{T}=I$. Also, $\hat{W} \hat{W}^{T}=W W^{T}+W^{\prime} W^{\prime T}$. For any vector $v$ in the span of the columns of $W$, we have $v=\hat{W} \hat{W}^{T} v=\left(W W^{T}+W^{\prime} W^{\prime T}\right) v=W W^{T} v$. In other words, $W$ acts as orthonormal matrix restricted to its image, and thus $W W^{T}$ acts as the identity. In particular, $W P=W W^{T} B=B$.

Our algorithm has access to $P^{\prime}$ as defined above rather than to $P$. The algorithm will form the product $W P^{\prime}$. But now it's clear from $W P=B$ that $W P_{\pi(j)}^{\prime}=\alpha_{j} B_{j}$. Thus the algorithm will recover $B$ up to sign and permutation. 


\subsection{Diagonalizability and robust analysis}

In applications of our tensor decomposition algorithm, we do not have access to the true underlying tensors $T_{\mu}$ and $T_{\lambda}$ but rather slightly perturbed versions. We prove now that under suitably small perturbations $R_{\mu}$ and $R_{\lambda}$, we are able to recover the correct rank 1 components with good accuracy. The statement of the robust version of this theorem closely follows that of the exact version: we merely need to add some assumptions on the magnitude of the perturbations relative to the quotients $\mu_{i} / \lambda_{i}$ in conditions 4 and 5 .

Theorem 5.4. Let $M_{\mu}, M_{\lambda} \in \mathbb{C}^{p \times p}$ such that

$$
M_{\mu}=B \operatorname{diag}\left(\mu_{i}\right) B^{T}, \quad M_{\lambda}=B \operatorname{diag}\left(\lambda_{i}\right) B^{T},
$$

where $B \in \mathbb{R}^{p \times m}$, and $\mu, \lambda \in \mathbb{C}^{m}$ for some $m \leq p$. For error matrices $R_{\mu}, R_{\lambda} \in \mathbb{C}^{p \times p}$, let $M_{\mu}+R_{\mu}$ and $M_{\lambda}+R_{\lambda}$ be perturbed versions of $M_{\mu}$ and $M_{\lambda}$. Let $0<\epsilon<1$. Suppose that the following conditions and data are given:

1. For each column $B_{i} \in \mathbb{R}^{m}$ of $B,\left\|B_{i}\right\|_{2}=1$.

2. $\sigma_{m}(B)>0$.

3. $\mu_{i}, \lambda_{i} \neq 0$ for all $i,\left|\frac{\mu_{i}}{\lambda_{i}}-\frac{\mu_{j}}{\lambda_{j}}\right| \geq \Omega>0$ for all $i \neq j$.

4. $0<K_{L} \leq\left|\mu_{i}\right|,\left|\lambda_{i}\right| \leq K_{U}$.

5. $\left\|R_{\mu}\right\|_{F},\left\|R_{\lambda}\right\|_{F} \leq K_{1} \leq \frac{\epsilon K_{L}^{2} \sigma_{m}(B)^{3}}{2^{11} \kappa(B)^{3} K_{U} m^{2}} \min (\Omega, 1)$.

Then Diagonalize applied to $M_{\mu}+R_{\mu}$ and $M_{\lambda}+R_{\lambda}$ outputs $\tilde{B}$ such that there exists a permutation $\pi:[m] \rightarrow[m]$ and phases $\alpha_{j}$ (a phase $\alpha$ is a scalar in $\mathbb{C}$ with $|\alpha|=1$ ) such that

$$
\left\|B_{j}-\alpha_{j} \tilde{B}_{\pi(j)}\right\| \leq \epsilon .
$$

The running time of the algorithm is poly $\left(p, \frac{1}{\Omega}, \frac{1}{K_{L}}, \frac{1}{\sigma_{\min }(B)}, \frac{1}{\epsilon}\right)$.

Proof. We begin with an informal outline of the proof. We basically implement the proof for the exact case, however because of the perturbations, various equalities now are true only approximately and this leads to somewhat lengthy and technical details, but the intuitive outline remains the same as for the exact case.

The algorithm constructs an orthonormal basis of the left singular space of $\bar{M}_{\mu}:=M_{\mu}+R_{\mu}$; denote by $Y$ the matrix with this basis as its columns. The fact that $\bar{M}_{\mu}$ is close to $M_{\mu}$ gives by Wedin's theorem (Theorem 8.2) that the left singular spaces of $\bar{M}_{\mu}$ and $M_{\mu}$ are close. More specifically, this means that there are two matrices, $W$ with columns forming an orthonormal basis for the left singular space of $M_{\mu}$, and $X$ with columns forming an orthonormal basis for the left singular space of $\bar{M}_{\mu}$ such that $W$ and $X$ are close in the entrywise sense. This implies that $W^{T} B$ and $X^{T} B$ are close. This can be used to show that under appropriate conditions $X^{T} B$ is nonsingular. Now using the fact that the columns of $Y$ and of $X$ span the same space, it follows that $\bar{P}:=Y^{T} B$ is nonsingular. In the next step, we show by virtue of $\left\|R_{\mu}\right\|$ being small that the matrix $Y^{T} \bar{M}_{\mu} Y$ constructed by the algorithm is close to $\bar{P} \operatorname{diag}\left(\mu_{i}\right) \bar{P}^{T}$ where the $\mu_{i}$ are the eigenvalues of $M_{\mu}$; and similarly for $Y^{T} \bar{M}_{\lambda} Y$. We then show that $\left(Y^{T} \bar{M}_{\mu} Y\right)\left(Y^{T} \bar{M}_{\lambda} Y\right)^{-1}$ is diagonalizable and the diagonalization provides a matrix $\tilde{P}$ close to $\bar{P}$, and so $\tilde{B}=Y \tilde{P}$ gives the columns of $B$ up to phase factors and permutation and small error.

A note on the running time. Algorithm Diagonalize uses SVD and eigenvector decomposition of diagonalizable (but not normal) matrices as subroutines. There are well-known algorithms for these as discussed earlier. The outputs of these algorithms are not exact and have a quantifiable error: The computation of SVD of $M \in \mathbb{C}^{n \times n}$ within error $\epsilon$ (for any reasonable notion of error, say $\left\|M-V \Sigma U^{T}\right\|_{F}$ where $V \Sigma U^{T}$ is the SVD output by the algorithm on input $M)$ can be done in time poly $\left(n, \frac{1}{\epsilon}, \frac{1}{\sigma_{\min }(M)}\right)$. Similarly, for the eigenvector decomposition of a diagonalizable matrix $M \in \mathbb{C}^{n \times n}$ with eigenvalues $\left|\lambda_{i}-\lambda_{j}\right| \geq \Omega>0$ for $i \neq j$, we can compute the decomposition within error $\epsilon$ in time $\operatorname{poly}\left(n, \frac{1}{\Omega}, \frac{1}{\epsilon}, \frac{1}{\min _{i}\left|\lambda_{i}\right|}\right)$. 
In the analysis below, we ignore the errors from these computations as they can be controlled and will be of smaller order than the error from the main analysis. This can be made rigorous but we omit the details in the interest of brevity. Combining the running time of the two subroutines one can check easily that the overall running time is what is claimed in the statement of the theorem.

We now proceed with the formal proof. The proof is broken into 7 steps.

Step 1. $W^{T} B \approx X^{T} B$.

Let $\bar{M}_{\mu}:=M_{\mu}+R_{\mu}$ and $\bar{M}_{\lambda}:=M_{\lambda}+R_{\lambda}$. Now the fact that $\left\|R_{\mu}\right\|_{F}$ is small implies by Wedin's theorem (Theorem 8.2) that the left singular spaces of $M_{\mu}$ and $\bar{M}_{\mu}$ are close: Specifically, by Theorem IV.1.8 in [16] about canonical angles between subspaces, we have: There exists an orthonormal basis of the left singular space of $M_{\mu}$ (given by the columns $w_{1}, \ldots w_{m}$ of $W \in \mathbb{C}^{p \times m}$ ) and an orthonormal basis of the left singular space of $\bar{M}_{\mu}$ (given by the columns $x_{1}, \ldots, x_{m}$ of $X \in \mathbb{C}^{p \times m}$ ) such that

$$
x_{j}=c_{j} w_{j}+s_{j} z_{j}, \text { for all } j,
$$

where $0 \leq c_{1} \leq \ldots \leq c_{m} \leq 1$, and $1 \geq s_{1} \geq \ldots \geq s_{m} \geq 0$, and $c_{j}^{2}+s_{j}^{2}=1$ for all $j$; vectors $w_{1}, \ldots, w_{m} ; z_{1}, \ldots, z_{m}$ form an orthonormal basis. (For the last condition to hold we need $p \geq 2 m$. A similar representation can be derived when this condition does not hold and the following computation will still be valid. We omit full discussion of this other case for brevity; in any case, we could arrange so that $p \geq 2 m$ without any great penalty in the parameters achieved.) We now apply Wedin's theorem 8.2 to $M_{\mu}$ and $\bar{M}_{\mu}$ to upper bound $s_{j}$. To this end, first note that by Claim 10.4 we have $\sigma_{m}\left(M_{\mu}\right) \geq K_{L} \sigma_{m}(B)^{2}$; and second, by Weyl's inequality for singular values (Lemma 8.3) we have $\left|\sigma_{j}\left(\bar{M}_{\mu}\right)-\sigma_{j}\left(M_{\mu}\right)\right| \leq \sigma_{1}\left(R_{\mu}\right) \leq K_{1}$ for all $j$. Thus in Theorem 8.2 with $\Sigma_{1}$ corresponding to non-zero singular values of $M_{\mu}$, we have $\max \sigma\left(\Sigma_{2}\right)=0$. And we can choose a corresponding conformal SVD of $\bar{M}_{\mu}$ so that $\min \sigma\left(\bar{\Sigma}_{1}\right) \geq K_{L} \sigma_{m}(B)^{2}-K_{1}$. Which gives, $\|\sin \Phi\|_{2} \leq K_{1} /\left(K_{L} \sigma_{m}(B)^{2}-K_{1}\right)=: K_{2}$, where $\Phi$ is the matrix of canonical angles between colspan $(W)$ and colspan $(X)$. Thus we have

$$
s_{j} \leq K_{2},
$$

for all $j$.

Now we can show that $X^{T} B$ is close to $W^{T} B$ : The $(i, j)^{\prime}$ th entry of $W^{T} B-X^{T} B$ is $\left(1-c_{i}\right) w_{i}^{T} b_{j}-s_{i} z_{i}^{T} b_{j}$. Using (15) and $\left\|w_{i}\right\|,\left\|b_{j}\right\|,\left\|z_{i}\right\| \leq 1$, we have

$$
\left(1-c_{i}\right) w_{i}^{T} b_{j}-s_{i} z_{i}^{T} b_{j} \leq s_{i}^{2}+s_{i} \leq 2 K_{2} .
$$

And so $\left\|W^{T} B-X^{T} B\right\|_{F} \leq 2 m^{2} K_{2}$. Hence by Lemma 8.3 we have $\left|\sigma_{j}\left(W^{T} B\right)-\sigma_{j}\left(X^{T} B\right)\right| \leq 2 m^{2} K_{2}$ for all $j$.

Step 2. $\quad \bar{P}:=Y^{T} B$ is full rank.

The singular values of $W^{T} B$ are the same as those of $B$. Briefly, this is because $W^{T}$ acts as an isometry on colspan $(B)$. Also observe that the singular values of $Y^{T} B$ are the same as those of $X^{T} B$. Briefly, this is because $Y^{T}$ and $X^{T}$ act as isometries on colspan $(X)=$ colspan $(Y)$. These two facts together with the closeness of the singular values of $W^{T} B$ and $X^{T} B$ as just shown imply that

$$
\left|\sigma_{j}(B)-\sigma_{j}\left(Y^{T} B\right)\right| \leq 2 m^{2} K_{2}
$$

for all $j$. Now using that $2 m^{2} K_{2}<\sigma_{m}(B) / 2$ (This follows by our condition 5 in the theorem giving an upper bound on $K_{1}: K_{1} \leq \epsilon \frac{K_{L}}{K_{U}} \frac{K_{L} \sigma_{m}(B)^{3}}{2^{11} \kappa(B)^{3} m^{2}}$ which gives $K_{1} \leq \frac{K_{L} \sigma_{m}(B)^{3}}{8 m^{2}}$. This in turn implies $2 m^{2} K_{2}<\sigma_{m}(B) / 2$ using $\sigma_{m}(B) \leq 1$; we omit easy verification.) we get that $\sigma_{m}\left(Y^{T} B\right)>0$ and hence $Y^{T} B$ is full rank. We note some consequences of (16) for use in later steps:

$$
\kappa(\bar{P}) \leq 4 \kappa(B) .
$$


This follows from $\kappa(\bar{P}) \leq \frac{\sigma_{1}(B)+2 m^{2} K_{2}}{\sigma_{m}(B)-2 m^{2} K_{2}} \leq 4 \kappa(B)$, because $2 m^{2} K_{2}<\sigma_{m}(B) / 2$.

$$
\begin{gathered}
\sigma_{m}(\bar{P}) \leq \sigma_{m}(B)+2 m^{2} K_{2}<2 \sigma_{m}(B) . \\
\sigma_{m}(\bar{P}) \geq \sigma_{m}(B)-2 m^{2} K_{2}<\sigma_{m}(B) / 2 .
\end{gathered}
$$

Step 3. $Y^{T} \bar{M}_{\mu} Y \approx \bar{P} \operatorname{diag}\left(\mu_{i}\right) \bar{P}^{T}$ and $Y^{T} \bar{M}_{\lambda} Y \approx \bar{P} \operatorname{diag}\left(\lambda_{i}\right) \bar{P}^{T}$.

More precisely, let $E_{\mu}:=Y^{T} \bar{M}_{\mu} Y-\bar{P} \operatorname{diag}\left(\mu_{i}\right) \bar{P}^{T}$, then $\left\|E_{\mu}\right\|_{F} \leq m^{2}\left\|R_{\mu}\right\|_{F}$; and similarly for $\bar{M}_{\lambda}, E_{\lambda}:=$ $Y^{T} \bar{M}_{\lambda} Y-\bar{P} \operatorname{diag}\left(\lambda_{i}\right) \bar{P}^{T}$. The proof is short: We have $Y^{T} \bar{M}_{\mu} Y=Y^{T}\left(M_{\mu}+R_{\mu}\right) Y=Y^{T} M_{\mu} Y+Y^{T} R_{\mu} Y=$ $\bar{P} \operatorname{diag}\left(\mu_{i}\right) \bar{P}^{T}+Y^{T} R_{\mu} Y$. Hence $\left\|E_{\mu}\right\|_{F}=\left\|Y^{T} R_{\mu} Y\right\|_{F} \leq\left\|R_{\mu}\right\|_{F}$.

Step 4. $\left(Y^{T} \bar{M}_{\mu} Y\right)\left(Y^{T} \bar{M}_{\lambda} Y\right)^{-1}$ is diagonalizable.

This is because Theorem5.5 is applicable to $\tilde{N}:=\left(Y^{T} \bar{M}_{\mu} Y\right)\left(Y^{T} \bar{M}_{\lambda} Y\right)^{-1}=\left(\bar{P} \operatorname{diag}\left(\mu_{i}\right) \bar{P}^{T}+E_{\mu}\right)\left(\bar{P} \operatorname{diag}\left(\lambda_{i}\right) \bar{P}^{T}+\right.$ $\left.E_{\lambda}\right)^{-1}$ : using $\left\|E_{\mu}\right\|_{F} \leq\left\|R_{\mu}\right\|_{F}$, the two condition to verify are

- $\frac{6 \kappa(\bar{P})^{3} m K_{U}}{K_{L}^{2} \sigma_{m}(P)^{2}} K_{1} \leq \Omega$.

This follows from our condition 5 using (17), (19) and $\sigma_{m}(B) \leq 1$.

- $K_{1} \leq \sigma_{m}(\bar{P})^{2} K_{L} / 2$.

This also follows from condition 5, using (18) and $\epsilon \leq 1$.

Hence $\tilde{N}$ is diagonalizable: $\tilde{N}=\tilde{P} \operatorname{diag}\left(\tilde{\gamma}_{i}\right) \tilde{P}^{-1}$.

Step 5. The eigenvalues of $\tilde{N}$ are close to the eigenvalues of $\bar{P} \operatorname{diag}\left(\mu_{i} / \lambda_{i}\right) \bar{P}^{T}$. This follows from our application of Theorem [5.5in the previous step (specifically from (24) ) and gives a permutation $\pi:[\mathrm{m}] \rightarrow[\mathrm{m}]$ such that

$$
\left|\frac{\mu_{i}}{\lambda_{i}}-\tilde{\gamma}_{\pi(i)}\right|<\Omega / 2
$$

where the $\tilde{\gamma}_{i}$ are the eigenvalues of $\tilde{N}$.

In the next step we show that there exist phases $\alpha_{i}$ such that $\tilde{P}^{\pi, \alpha}:=\left[\alpha_{1} \tilde{P}_{\pi(1)}, \alpha_{2} \tilde{P}_{\pi(2)}, \ldots, \alpha_{m} \tilde{P}_{\pi(m)}\right]$ is close to $\bar{P}$.

Step 6. $\bar{P}$ is close to $\tilde{P}$ up to sign and permutation of columns.

We upper bound the angle $\theta$ between the corresponding eigenpairs $\left(\frac{\mu_{j}}{\lambda_{j}}, \bar{P}_{j}\right)$ and $\left(\tilde{\gamma}_{\pi(j)}, \tilde{P}_{\pi(j)}\right)$ of $N:=$ $\bar{P} \operatorname{diag}\left(\mu_{i} / \lambda_{i}\right) \bar{P}^{-1}$ and $\tilde{N}$. Theorem 8.7 (a generalized version of the $\sin (\theta)$ eigenspace perturbation theorem for diagonalizable matrices) applied to $N$ and $\tilde{N}$ gives (with the notation derived from Theorem 8.7)

$$
\sin \theta \leq \kappa\left(Z_{2}\right) \frac{\left\|\left(N-\tilde{\gamma}_{\pi(j)} I\right) \tilde{P}_{\pi(j)}\right\|_{2}}{\min _{i}\left|\left(N_{2}\right)_{i i}-\tilde{\gamma}_{\pi(j)}\right|} .
$$

To bound the RHS above, we will estimate each of the three terms. The first term:

$$
\kappa\left(Z_{2}\right) \leq \kappa\left(\bar{P}^{-1}\right)=\kappa(\bar{P}) \leq 4 \kappa(B),
$$

where for the first inequality we used that the condition number of a submatrix can only be smaller [63]; the second inequality is (17). 
Setting Err :=N-N, we bound the second term:

$$
\begin{aligned}
\left\|\left(N-\tilde{\gamma}_{\pi(j)} I\right) \tilde{P}_{\pi(j)}\right\|_{2} & =\left\|\left(\tilde{N}-\tilde{\gamma}_{\pi(j)} I\right) \tilde{P}_{\pi(j)}+\operatorname{Err} \tilde{P}_{\pi(j)}\right\|_{2} \\
& =\left\|\operatorname{Err} \tilde{P}_{\pi(j)}\right\|_{2} \\
& \leq\|\operatorname{Err}\|_{2} \\
& \left.\leq \kappa(\bar{P})^{2} \cdot \frac{K_{U}}{K_{L}} \cdot 2 m \cdot \frac{K_{1}}{\sigma_{m}(\bar{P})^{2} K_{L}} \quad \text { (by (23) }\right) \text { in Theorem 5.5) } \\
& \leq 2^{6} \kappa(B)^{2} m \frac{K_{U}}{K_{L}} \frac{K_{1}}{\sigma_{m}(B)^{2} K_{L}} \quad \text { (using (17), (18) ). }
\end{aligned}
$$

And lastly, the third term:

$$
\begin{aligned}
\min _{i}\left|\left(N_{2}\right)_{i i}-\tilde{\gamma}_{\pi(j)}\right| & \geq \min _{i: i \neq j}\left|\frac{\mu_{i}}{\lambda_{i}}-\frac{\mu_{j}}{\lambda_{j}}\right|-\left|\frac{\mu_{j}}{\lambda_{j}}-\tilde{\gamma}_{\pi(j)}\right| \\
& \geq \Omega-\kappa(\bar{P})\|\operatorname{Err}\|_{2} \text { (using Lemma 8.5) } \\
& \geq \Omega-2^{9} \kappa(B)^{3} m \frac{K_{U}}{K_{L}} \frac{K_{1}}{\sigma_{m}(B)^{2} K_{L}} \quad \text { (using (20) and (17)). }
\end{aligned}
$$

To abbreviate things a bit, let's set $\epsilon^{\prime}:=2^{9} \kappa(B)^{3} \frac{K_{U}}{K_{L}} m \frac{K_{1}}{\sigma_{m}(B)^{2} K_{L}}$. Then, putting things together we get

$$
\sin (\theta) \leq \frac{\epsilon^{\prime}}{\Omega-\epsilon^{\prime}}
$$

Now using the fact that the columns of $\tilde{P}$ and $\bar{P}$ are unit length implies that there exist phases $\alpha_{i}$ such that

$$
\left\|\alpha_{j} \tilde{P}_{\pi(j)}-\bar{P}_{j}\right\|_{2} \leq \frac{\epsilon^{\prime}}{\Omega-\epsilon^{\prime}}
$$

Step 7. $Y \tilde{P}$ gives $B$ approximately and up to phase factors and permutation of columns.

This follows from two facts: (1) $\tilde{P}^{\pi, \alpha} \approx \bar{P}$, so $Y \tilde{P}^{\pi, \alpha} \approx Y \bar{P}$ (we will prove this shortly); and (2) $Y \bar{P}=Y Y^{T} B$ (follows by the definition of $\bar{P}$ ). Now note that the operator $Y Y^{T}$ is projection to colspan $(Y)$; since the angle between colspan $(Y)$ and colspan $(B)$ is small as we showed in Step 1, we get that $Y Y^{T} B \approx B$.

Formally, we have

$$
\left\|Y \alpha_{j} \tilde{P}_{\pi(j)}-Y \bar{P}_{j}\right\|_{2} \leq\|Y\|_{2}\left\|\alpha_{j} \tilde{P}_{\pi(j)}-\bar{P}_{j}\right\|_{2} \leq \frac{\epsilon^{\prime}}{\Omega-\epsilon^{\prime}}
$$

using (21). And

$$
\left\|b_{j}-Y Y^{T} b_{j}\right\|_{2} \leq K_{2}
$$

where the last inequality used that the sine of the angle between colspan $(Y)$ and colspan $(W)=$ colspan $(B)$ is at most $K_{2}$ as proved in Step 1.

Putting these together we get

$$
\left\|Y \alpha_{j} \tilde{P}_{\pi(j)}-b_{j}\right\|_{2} \leq\left\|b_{j}-Y Y^{T} b_{j}\right\|_{2}+\left\|Y \alpha_{j} \tilde{P}_{\pi(j)}-Y \bar{P}_{j}\right\|_{2} \leq \frac{\epsilon^{\prime}}{\Omega-\epsilon^{\prime}}+K_{2} .
$$

Letting $\tilde{B}=Y \tilde{P}$ gives

$$
\left\|\alpha_{j} \tilde{B}_{\pi(j)}-b_{j}\right\|_{2} \leq \frac{\epsilon^{\prime}}{\Omega-\epsilon^{\prime}}+K_{2} \leq \epsilon
$$

The last inequality follows from our condition 5 which implies that $\frac{\epsilon^{\prime}}{\Omega-\epsilon^{\prime}} \leq \epsilon / 2$ and $K_{2} \leq \epsilon / 2$. 
Theorem 5.5 (Diagonalizability of perturbed matrices). Let $N_{\mu}, N_{\lambda} \in \mathbb{C}^{m \times m}$ be full rank complex matrices such that $N_{\mu}=Q \operatorname{diag}\left(\mu_{i}\right) Q^{T}, N_{\lambda}=Q \operatorname{diag}\left(\lambda_{i}\right) Q^{T}$ for some $Q \in \mathbb{R}^{m \times m}$ and $\mu, \lambda \in \mathbb{C}^{m}$. Suppose we also have the following conditions and data:

1. $0<K_{L} \leq\left|\mu_{i}\right|,\left|\lambda_{i}\right| \leq K_{U}$.

2. $\left|\mu_{i} / \lambda_{i}-\mu_{j} / \lambda_{j}\right|>\Omega>0$ for all pairs $i \neq j$.

3. $0<K<1$ and $E_{\mu}, E_{\lambda} \in \mathbb{C}^{m \times m}$ such that $\left\|E_{\mu}\right\|_{F},\left\|E_{\lambda}\right\|_{F} \leq K$.

4. $6 \kappa(Q)^{3} \cdot \frac{K_{U}}{K_{L}} \cdot m \cdot \frac{K}{\sigma_{m}(Q)^{2} K_{L}} \leq \Omega$.

5. $K \leq \sigma_{m}(Q)^{2} K_{L} / 2$.

Then $\left(N_{\mu}+E_{\mu}\right)\left(N_{\lambda}+E_{\lambda}\right)^{-1}$ is diagonalizable and hence has $n$ eigenvectors.

Proof. Defining $F_{\mu}:=\left(Q \operatorname{diag}\left(\mu_{i}\right) Q^{T}\right)^{-1} E_{\mu}$, and similarly $F_{\lambda}$, we have

$$
\begin{aligned}
\left(N_{\mu}+E_{\mu}\right)\left(N_{\lambda}+E_{\lambda}\right)^{-1} & =\left(Q \operatorname{diag}\left(\mu_{i}\right) Q^{T}+E_{\mu}\right)\left(Q \operatorname{diag}\left(\lambda_{i}\right) Q^{T}+E_{\lambda}\right)^{-1} \\
& =Q \operatorname{diag}\left(\mu_{i}\right) Q^{T}\left(I+F_{\mu}\right)\left(I+F_{\lambda}\right)^{-1}\left(Q \operatorname{diag}\left(\lambda_{i}\right) Q^{T}\right)^{-1} \\
& =Q \operatorname{diag}\left(\mu_{i}\right) Q^{T}\left(I+F_{\mu}\right)\left(I+G_{\lambda}\right)\left(Q \operatorname{diag}\left(\lambda_{i}\right) Q^{T}\right)^{-1} \\
& =Q \operatorname{diag}\left(\mu_{i} / \lambda_{i}\right) Q^{-1}+\operatorname{Err.}
\end{aligned}
$$

In (22) above $G_{\lambda}=\left(I+F_{\lambda}\right)^{-1}-I$; hence by Claim 10.5 (which is applicable because $\left\|F_{\lambda}\right\|_{F} \leq \frac{K}{\sigma_{m}(Q)^{2} K_{L}} \leq$ $1 / 2$, by our assumption) we have $\left\|G_{\lambda}\right\|_{F} \leq(m+1)\left\|F_{\lambda}\right\|_{F}$. The norm of Err then satisfies

$$
\begin{aligned}
\|\operatorname{Err}\|_{F} & \leq \frac{\sigma_{1}(Q)^{2}}{\sigma_{m}(Q)^{2}} \cdot \frac{K_{U}}{K_{L}}\left(\left\|F_{\mu}\right\|_{F}+(m+1)\left\|F_{\lambda}\right\|_{F}+(m+1)\left\|F_{\mu}\right\|_{F} \cdot\left\|F_{\lambda}\right\|_{F}\right) \\
& \leq \kappa(Q)^{2} \cdot \frac{K_{U}}{K_{L}} \cdot 2 m \cdot \frac{K}{\sigma_{m}(Q)^{2} K_{L}} .
\end{aligned}
$$

Now note that $3 \kappa(Q)\|\operatorname{Err}\|_{2} \leq 6 \kappa(Q)^{3} \cdot \frac{K_{U}}{K_{L}} \cdot m \cdot \frac{K}{\sigma_{m}(Q)^{2} K_{L}} \leq \Omega$ by our assumption and so Lemma 8.5 is applicable with matrices $Q \operatorname{diag}\left(\mu_{i} / \lambda_{i}\right) Q^{-1}, Q$, and Err playing the roles of $A, X$, and $E$, resp. Lemma 8.5 gives us a permutation $\pi:[m] \rightarrow[m]$ such that

$$
\left|\nu_{\pi(i)}\left(Q \operatorname{diag}\left(\mu_{i} / \lambda_{i}\right) Q^{-1}+\operatorname{Err}\right)-\nu_{i}\left(Q \operatorname{diag}\left(\mu_{i} / \lambda_{i}\right) Q^{-1}\right)\right| \leq \kappa(Q)\|\operatorname{Err}\|_{2}<\Omega / 2,
$$

where $\nu_{i}(M)$ denotes an eigenvalue of $M$.

Hence all the eigenvalues of $\left(N_{\mu}+E_{\mu}\right)\left(N_{\lambda}+E_{\lambda}\right)^{-1}$ are distinct. By Lemma 8.6, it has $n$ linearly independent eigenvectors $\left\{v_{1}, \ldots, v_{n}\right\}$.

\section{Underdetermined ICA}

In this section we give our algorithm for the underdetermined ICA problem and analyze it. In the underdetermined case, there are more independent source variables than there are measurements, thus $A$ has fewer rows than columns. We have to be more careful about fixing the normalization and scaling of the model than in the fully determined case where isotropic position provides a convenient normalization for $x, A$ and $s$.

Problem 1 (Underdetermined ICA). Fix $n, m \in \mathbb{N}$ such that $n \leq m$. We say that $x \in \mathbb{R}^{n}$ is generated by an underdetermined ICA model if $x=$ As for some fixed matrix $A \in \mathbb{R}^{n \times m}$ where A has full row rank and $s \in \mathbb{R}^{m}$ is a fully independent random vector. In addition, we fix the normalization so that each column $A_{i}$ has unit norm. The problem is to recover the columns of $A$ from independent samples $x$ modulo phase factors. 
Additional assumptions are needed for the essentially unique identifiability of this model. For example, suppose that columns $A_{i}$ and $A_{j}$ are parallel i.e., $A_{i}=c A_{j}$, then one could replace the variables $s_{i}$ and $s_{j}$ with $s_{i}+c s_{j}$ and 0 and the model would still be consistent. We introduce the following sufficient condition for identifiability: we require that the $m$ column vectors of $A^{\odot k}$ be linearly independent for some $k>0$ (smaller $k$ would be better for the efficiency of the algorithm). We make this quantitative by requiring that the $m$ 'th singular value satisfy $\sigma_{m}\left(A^{\odot k}\right)>0$.

Our approach to the underdetermined ICA problem is to apply our tensor decomposition to a pair of carefully-chosen tensors that arise from the distribution. The tensors we use are the derivative tensors of the second charateristic function $\psi_{x}(u)=\log \left(\mathbb{E}\left(e^{i u^{T} x}\right)\right)$.

This method generalises the fourth moment methods for ICA where one computes the local optima of the following quartic form:

$$
f(u)=\mathbb{E}\left(\left(x^{T} u\right)^{4}\right)-3 \mathbb{E}\left(\left(x^{T} u\right)^{2}\right)^{2} .
$$

An equivalent formulation of this is to consider the tensor $T \in \mathbb{R}^{n \times n \times n \times n}$ which represents this quartic form (just as in the matrix case where symmetric matrices represent quadratic forms, symmetric tensors of order 4 represent quartic forms). Let us denote our overall tensor representing $f(u)$ by $T$ where $f(u)=T(u, u, u, u)$. By a relatively straightforward calculation, one can verify that $T(u, u, u, u)$ is the fourth derivative of the second characteristic function of $x$ evalauted at 0 :

$$
T=D_{u}^{4} \psi_{x}(0) .
$$

On the other hand, one can also verify that $T$ has the following decomposition (see for example [4):

$$
T=\sum_{j=1}^{m}\left(\mathbb{E}\left(s_{i}^{4}\right)-3 \mathbb{E}\left(s_{i}^{2}\right)^{2}\right) A_{i} \otimes A_{i} \otimes A_{i} \otimes A_{i}
$$

So in fact, one can view the fourth moment tensor methods as performing the tensor decomposition of only one tensor - the fourth derivative of $\psi$ evaluated at 0 !

Our method also generalises the algorithm we gave for the fully determined case in Section 4 We can view that case as simply being the second derivative version of the more general algorithm. The techniques used in this section are generalisations and refinements of those used in the fully determined case, though replacing the easy matrix decomposition arguments with the corresponding (harder) tensor arguments.

A property of the second characteristic function that is central for our algorithm is that the second characteristic function of a random vector with independent components factorizes into the sum of the second characteristic functions of each component:

$$
\log \left(\mathbb{E}\left(e^{i u^{T} s}\right)\right)=\sum_{j=1}^{m} \log \left(\mathbb{E}\left(e^{i u_{j} s_{j}}\right)\right),
$$

and now every mixed partial derivative (with respect to $u_{j}$ and $u_{j^{\prime}}$ ) is 0 , as each term in the sum depends only on one component of $u$. Taking the derivative tensor will result in a diagonal tensor where the offdiagonal terms are all 0 . In the case when we're interested in $x=A s$, we simply need to perform the change of basis via $A$ very carefully for the derivative tensors via the chain rule. One could also try to perform this analysis with the moment generating function $\mathbb{E}\left(e^{u^{T} x}\right)$ without the complex phase. The difficulty here is that the moment generating functions exists only if all moments of $x$ exist, and thus a moment generating function approach would not be able to deal with heavy tailed distributions. Moreover, using a real exponential leads us to estimate exponentially large quantities from samples, and it is difficult to get good bounds on the sample complexity. Using the complex exponential avoids these problems as all quantities have modulus 1.

We will then apply our tensor decomposition framework: as before we show that the eigenvalues of the flattened derivative tensors are well spaced in Section 6.3. We then study the sample complexity in Section 6.4 and assembling these components in Section 6.5. 


\subsection{Algorithm}

For underdetermined ICA we compute the higher derivative tensors of the second characteristic function $\psi_{x}(u)=\log \left(\phi_{x}(u)\right)$ at two random points and run the tensor decomposition algorithm from the previous section.

\section{Underdetermined $\operatorname{ICA}(\sigma)$}

1. (Compute derivative tensor) Pick independent random vectors $\alpha, \beta \sim$ $N\left(0, \sigma^{2} I_{n}\right)$. For even $d$, estimate the $d^{t h}$ derivative tensors of $\psi_{x}(u)$ at $\alpha$ and $\beta$ as $T_{\alpha}=D_{u}^{d} \psi_{x}(\alpha)$ and $T_{\beta}=D_{u}^{d} \psi_{x}(\beta)$.

2. (Tensor decomposition) Run Tensor Decomposition $\left(T_{\alpha}, T_{\beta}\right)$.

To estimate the $2 d^{\text {th }}$ derivative tensor of $\psi_{x}(u)$ empirically, one simply writes down the expression for the derivative tensor, and then estimates each entry from samples using the naive estimator.

More precisely, we can use

$$
\frac{\partial \phi(u)}{\partial u_{i}}=\mathbb{E}\left(\left(i x_{i}\right) e^{i u^{T} x}\right) .
$$

This states that differentiation in the Fourier space is equivalent to multiplication in the original space, thus it suffices to estimate monomials of $x$ reweighted by complex exponentials. To estimate the $d^{\text {th }}$ derivative tensor of $\log (\phi(u))$ empirically, one simply writes down the expression for the derivative tensor, and then estimates each entry from samples using the naive estimator. Note that the derivatives can be somewhat complicated, for example, at fourth order we have

$$
\begin{aligned}
& {\left[D^{4} \psi_{u}\right]_{i_{1}, i_{2}, i_{3}, i_{4}}} \\
& =\frac{1}{\phi(u)^{4}}\left[\mathbb{E}\left(\left(i x_{i_{1}}\right)\left(i x_{i_{2}}\right)\left(i x_{i_{3}}\right)\left(i x_{i_{4}}\right) \exp \left(i u^{T} x\right)\right) \phi(u)^{3}\right. \\
& \quad-\mathbb{E}\left(\left(i x_{i_{2}}\right)\left(i x_{i_{3}}\right)\left(i x_{i_{4}}\right) \exp \left(i u^{T} x\right)\right) \mathbb{E}\left(\left(i x_{i_{1}}\right) \exp \left(i u^{T} x\right)\right) \phi(u)^{2} \\
& \quad-\mathbb{E}\left(\left(i x_{i_{2}}\right)\left(i x_{i_{3}}\right) \exp \left(i u^{T} x\right)\right) \mathbb{E}\left(\left(i x_{i_{1}}\right)\left(i x_{i_{4}}\right) \exp \left(i u^{T} x\right)\right) \phi(u)^{2} \\
& \quad-\mathbb{E}\left(\left(i x_{i_{2}}\right)\left(i x_{i_{4}}\right) \exp \left(i u^{T} x\right)\right) \mathbb{E}\left(\left(i x_{i_{1}}\right)\left(i x_{i_{3}}\right) \exp \left(i u^{T} x\right)\right) \phi(u)^{2} \\
& \quad-\mathbb{E}\left(\left(i x_{i_{2}}\right) \exp \left(i u^{T} x\right)\right) \mathbb{E}\left(\left(i x_{i_{1}}\right)\left(i x_{i_{3}}\right)\left(i x_{i_{4}}\right) \exp \left(i u^{T} x\right)\right) \phi(u)^{2} \\
& \quad-\mathbb{E}\left(\left(i x_{i_{3}}\right)\left(i x_{i_{4}}\right) \exp \left(i u^{T} x\right)\right) \mathbb{E}\left(\left(i x_{i_{1}}\right)\left(i x_{i_{2}}\right) \exp \left(i u^{T} x\right)\right) \phi(u)^{2} \\
& \quad-\mathbb{E}\left(\left(i x_{i_{3}}\right) \exp \left(i u^{T} x\right)\right) \mathbb{E}\left(\left(i x_{i_{1}}\right)\left(i x_{i_{2}}\right)\left(i x_{i_{4}}\right) \exp \left(i u^{T} x\right)\right) \phi(u)^{2} \\
& \quad-\mathbb{E}\left(\left(i x_{i_{4}}\right) \exp \left(i u^{T} x\right)\right) \mathbb{E}\left(\left(i x_{i_{1}}\right)\left(i x_{i_{2}}\right)\left(i x_{i_{3}}\right) \exp \left(i u^{T} x\right)\right) \phi(u)^{2} \\
& \quad+2 \mathbb{E}\left(\left(i x_{i_{3}}\right)\left(i x_{i_{4}}\right) \exp \left(i u^{T} x\right)\right) \mathbb{E}\left(\left(i x_{i_{2}}\right) \exp \left(i u^{T} x\right)\right) \mathbb{E}\left(\left(i x_{i_{1}}\right) \exp \left(i u^{T} x\right)\right) \phi(u) \\
& \quad+2 \mathbb{E}\left(\left(i x_{i_{3}}\right) \exp \left(i u^{T} x\right)\right) \mathbb{E}\left(\left(i x_{i_{2}}\right)\left(i x_{i_{4}}\right) \exp \left(i u^{T} x\right)\right) \mathbb{E}\left(\left(i x_{i_{1}}\right) \exp \left(i u^{T} x\right)\right) \phi(u) \\
& \quad+2 \mathbb{E}\left(\left(i x_{i_{4}}\right) \exp \left(i u^{T} x\right)\right) \mathbb{E}\left(\left(i x_{i_{2}}\right)\left(i x_{i_{3}}\right) \exp \left(i u^{T} x\right)\right) \mathbb{E}\left(\left(i x_{i_{1}}\right) \exp \left(i u^{T} x\right)\right) \phi(u) \\
& \quad+2 \mathbb{E}\left(\left(i x_{i_{3}}\right) \exp \left(i u^{T} x\right)\right) \mathbb{E}\left(\left(i x_{i_{2}}\right) \exp \left(i u^{T} x\right)\right) \mathbb{E}\left(\left(i x_{i_{1}}\right)\left(i x_{i_{4}}\right) \exp \left(i u^{T} x\right)\right) \phi(u) \\
& \quad+2 \mathbb{E}\left(\left(i x_{i_{4}}\right) \exp \left(i u^{T} x\right)\right) \mathbb{E}\left(\left(i x_{i_{2}}\right) \exp \left(i u^{T} x\right)\right) \mathbb{E}\left(\left(i x_{i_{1}}\right)\left(i x_{i_{3}}\right) \exp \left(i u^{T} x\right)\right) \phi(u) \\
& \quad+2 \mathbb{E}\left(\left(i x_{i_{4}}\right) \exp \left(i u^{T} x\right)\right) \mathbb{E}\left(\left(i x_{i_{3}}\right) \exp \left(i u^{T} x\right)\right) \mathbb{E}\left(\left(i x_{i_{1}}\right)\left(i x_{i_{2}}\right) \exp \left(i u^{T} x\right)\right) \phi(u) \\
& \left.\quad-6 \mathbb{E}\left(\left(i x_{i_{1}}\right) \exp \left(i u^{T} x\right)\right) \mathbb{E}\left(\left(i x_{i_{2}}\right) \exp \left(i u^{T} x\right)\right) \mathbb{E}\left(\left(i x_{i_{3}}\right) \exp \left(i u^{T} x\right)\right) \mathbb{E}\left(\left(i x_{i_{4}}\right) \exp \left(i u^{T} x\right)\right)\right] .
\end{aligned}
$$

The salient points are described in Lemma 4.9 and Claim 4.10 there are at most $2^{d-1}(d-1)$ ! terms (counting multiplicities), and no term has combined exponents of $x_{i}$ in all it factors higher than $d$. We will give a rigorous analysis of the sampling error incurred in Section 6.4 


\subsection{Truncation error}

Lemma 6.1. Let $s=\left(s_{1}, \ldots, s_{m}\right) \in \mathbb{R}^{m}$ be a random vector with indpendent components each with finite $k$ absolute moments, and for $t \in \mathbb{R}^{m}$ let $\phi(t)=\mathbb{E}\left(e^{i t^{T} s}\right)$ be the associated characteristic function. Then for $k \geq 1$ and $i_{1}, \ldots i_{k} \in[m]$ we have

$$
\left|\partial_{i_{1}, \ldots, i_{k}} \log \phi(t)\right| \leq \frac{2^{k-1}(k-1) ! \max _{j \in[m]} \mathbb{E}\left(\left|s_{j}\right|^{k}\right)}{|\phi(t)|^{k}} .
$$

Proof. To compute the derivatives of $\log \phi(t)$ we proceed inductively with $\partial_{i_{1}} \log \phi(t)=\left(\partial_{i_{1}} \phi(t)\right) / \phi(t)$ as our base case. For $d<k$, write $\partial_{i_{1}, \ldots, i_{d}}(\log \phi)$ as $N_{d}(t) / \phi(t)^{d}$. Then we have

$$
\begin{aligned}
\partial_{i_{1}, \ldots, i_{d+1}} \log \phi(t) & =\partial_{i_{d+1}}\left(\frac{N_{d}(t)}{\phi(t)^{d}}\right) \\
& =\frac{\left(\partial_{i_{d+1}} N_{d}(t)\right) \phi(t)^{d}-N_{d}(t) d \phi(t)^{d-1} \partial_{i_{d+1}} \phi(t)}{\phi(t)^{2 d}} \\
& =\frac{\left(\partial_{i_{d+1}} N_{d}(t)\right) \phi(t)-d N_{d}(t) \partial_{i_{d+1}} \phi(t)}{\phi(t)^{d+1}} .
\end{aligned}
$$

We make the following claim about $N_{d}(t)$ :

Claim 6.2. The functions $N_{d}(t)$ is the sum of terms of the form $C_{S_{1}, \ldots, S_{d}} \partial_{S_{1}} \ldots \partial_{S_{d}} \phi(t)$ where multisets $S_{1}, \ldots, S_{d} \subseteq\left\{i_{1}, \ldots, i_{d}\right\}$ (this is a multiset) satisfy $S_{1} \cup \ldots \cup S_{d}=\left\{i_{1}, \ldots, i_{d}\right\}$, and $C_{S_{1}, \ldots, S_{d}}$ are integer coefficients with $\sum\left|C_{S_{1}, \ldots, S_{d}}\right| \leq 2^{d-1}(d-1)$ !.

Proof. The first part follows via induction on $d$ and (25). For the second part, let $T(d)$ denote $\sum\left|C_{S_{1}, \ldots, S_{d}}\right|$. Note that $T(1)=1$. Then by (25), we have $T(d+1) \leq d T(d)+d T(d)$, which gives $T(d) \leq 2^{d-1}(d-1)$ !.

Returning to the proof of Lemma 6.1, we observe that for any multiset $S$ with elements from $[\mathrm{m}]$ and size at most $k$, we have

$$
\left|\partial_{S} \phi(t)\right|=\left|\mathbb{E}\left(i^{|S|} s_{S} e^{i t^{T} s}\right)\right| \leq \mathbb{E}\left(\left|s_{S}\right|\right) .
$$

For $\ell \in[m]$, let $p_{\ell}$ be the number of times $\ell$ occurs in the multiset $\left\{i_{1}, \ldots, i_{d}\right\}$. For each term of $N_{d}(t)$ we have

$$
\begin{aligned}
\left|\prod_{j=1}^{d} \partial_{S_{j}} \phi\right| & =\prod_{j=1}^{d}\left|\partial_{S_{j}} \phi\right| \\
& \leq \prod_{j=1}^{d} \mathbb{E}\left(\left|s_{S_{j}}\right|\right) \\
& =\prod_{\ell=1}^{m} \mathbb{E}\left(\left|s_{\ell}\right|^{p_{\ell}}\right) \\
& \leq \prod_{\ell=1}^{m}\left(\mathbb{E}\left(\left|s_{\ell}\right|^{d}\right)\right)^{p_{\ell} / d} \\
& \leq \max _{\ell \in[m]} \mathbb{E}\left(\left|s_{\ell}\right|^{d}\right) .
\end{aligned}
$$

The second equality above uses the independence of the $s_{\ell}$, and the second inequality uses the first part of Fact 10.6

Thus $\left|N_{d}(t)\right| \leq 2^{(d-1)}(d-1) ! \max _{\ell \in[m]} \mathbb{E}\left(\left|s_{\ell}\right|^{d}\right)$, which when divided by $\phi(t)^{d}$ gives the required bound. 


\subsection{Eigenvalue spacings}

In this subsection we examine the anti-concentration of the diagonal entries $\psi_{i}^{(d)}\left(\left(A^{T} u\right)_{i}\right)$. The analysis has similarities to the fully-determined case but there are also some major differences: in the fully-determined case, $A_{i}^{T} u$ and $A_{j}^{T} u$ are independent Gaussians because the columns of $A$ are orthogonal by isotropic position (recall that we defined $A_{i}^{T}$ to mean $\left(A_{i}\right)^{T}$ ). We can not make $A$ an orthonormal matrix in the underdetermined case, so we have to exploit the more limited randomness. An additional complication is that we are working with anti-concentration of the quotients of such diagonal entries rather than the entries themselves.

Theorem 6.3. Let $s \in \mathbb{R}^{m}$ be a random vector with independent components. For $t \in \mathbb{R}^{m}$ and $d \in 2 \mathbb{N}$ let $\psi_{a}(t):=\log \mathbb{E}\left(e^{i t_{a} s_{a}}\right)$, and $g_{a}\left(t_{a}\right):=\frac{d^{d} \psi_{a}\left(t_{a}\right)}{d t_{a}^{d}}$ for all $a \in[m]$. Let $0<\delta$. Suppose that the following data and conditions are given:

1. $\mathbb{E}\left(s_{a}\right)=0, \mathbb{E}\left(s_{a}^{2}\right) \leq M_{2}$ and $\mathbb{E}\left(s_{a}^{d}\right) \leq M_{d}$ for $a \in[m]$ and $M_{2}<M_{d}$.

2. $k \geq 2$ and for all $a \in[m], k_{a} \in \mathbb{N}$ where $d<k_{a}<k$, such that $\left|\operatorname{cum}_{k_{a}}\left(s_{a}\right)\right| \geq \Delta$.

3. $\mathbb{E}\left(\left|s_{a}\right|^{k_{a}+1}\right) \leq M_{k}$ for $a \in[m]$ and $M_{2}<M_{k}$.

4. $A \in \mathbb{R}^{n \times m}$ be a full row rank matrix whose columns all have unit norm and $1-\left\langle A_{a}, A_{b}\right\rangle^{2} \geq L^{2}$ for all pairs of columns.

5. $u, v \sim N\left(0, \sigma^{2} I_{n}\right)$ sampled independently where

$$
\sigma \leq \min \left(1, \frac{1}{2 \sqrt{2 M_{2} \log 1 / q}}, \sigma^{\prime}\right)
$$

and

$$
\sigma^{\prime}=\Delta \frac{k-d+1}{k !}\left(\frac{3}{8}\right)^{k} \frac{1}{M_{k}}\left(\frac{L q \sqrt{2 \pi}}{4(k-d)}\right)^{k-d}\left(\frac{1}{\sqrt{2 \log 1 / q}}\right)^{k-d}
$$

and $0<q<1 / 3$. Then with probability at least $1-3\left(\begin{array}{c}m \\ 2\end{array}\right) q$ we have

$$
\left|\frac{g_{b}\left(A_{b}^{T} u\right)}{g_{b}\left(A_{b}^{T} v\right)}-\frac{g_{a}\left(A_{a}^{T} u\right)}{g_{a}\left(A_{a}^{T} v\right)}\right| \geq \Delta \frac{1}{(k-d) !(d-1) !}\left(\frac{3}{8}\right)^{d} \frac{1}{M_{d}}\left(\frac{\sigma L q \sqrt{2 \pi}}{4(k-d)}\right)^{(k-d)},
$$

for all distinct $a, b \in[m]$. (We count the small probability case where $g_{b}\left(A_{b}^{T} v\right)=0$ or $g_{a}\left(A_{a}^{T} v\right)=0$ as violating the event in (27).)

Proof. Fix $a \neq b \in[m]$ and show that the spacing in (27) is lower bounded for this pair with high probability. We will then take a union bound over all $\left(\begin{array}{c}m \\ 2\end{array}\right)$ pairs, which will give the desired result.

For random choice of $v$, the events

$$
g_{a}\left(A_{a}^{T} v\right) \neq 0 \text { and } g_{b}\left(A_{b}^{T} v\right) \neq 0
$$

have probability 1 . Thus in the following we will assume that these events are true.

We will need concentration of $\left(A_{a}^{T} u\right)$ and of $\left(A_{a}^{T} v\right)$.

$$
\operatorname{Pr}_{u \sim N\left(0, \sigma^{2}\right)}\left(\left|A_{a}^{T} u\right|>\tau\right) \leq \sqrt{\frac{2}{\pi}} \sigma^{2}\|r\|^{2} \frac{1}{\tau} e^{-\frac{\tau^{2}}{2 \sigma^{2}\|r\|^{2}}} \leq \sqrt{\frac{2}{\pi}} \sigma^{2} \frac{1}{\tau} e^{-\frac{\tau^{2}}{2 \sigma^{2}}},
$$


here the first inequality used Claim 10.3 and the second used the fact that $\|r\| \leq 1$. Substituting $\tau=$ $\sigma \sqrt{2 \log 1 / q}$ we get

$$
\operatorname{Pr}\left(\left|A_{a}^{T} u\right| \leq \sigma \sqrt{2 \log 1 / q}\right) \geq 1-\frac{\sigma q}{\sqrt{\pi \log 1 / q}} \geq 1-\frac{q}{\sqrt{\pi \log 1 / q}} .
$$

By the union bound we have

$$
\operatorname{Pr}\left(\left|A_{a}^{T} u\right|,\left|A_{a}^{T} v\right| \leq \sigma \sqrt{2 \log 1 / q}\right) \geq 1-\frac{2 q}{\sqrt{\pi \log 1 / q}} .
$$

In the sequel we will assume that the event in the previous expression happens.

Under the assumption that $\left|A_{a}^{T} v\right| \leq \sigma \sqrt{2 \log 1 / q}$ we have

$$
\left|g_{a}\left(A_{a}^{T} v\right)\right|=\left|\psi^{(d)}\left(A_{a}^{T} v\right)\right| \leq \frac{2^{d-1}(d-1) ! M_{d}}{(3 / 4)^{d}}
$$

where to upper bound $\left|\psi^{(d)}\left(A_{a}^{T} u\right)\right|$ we used Lemma 4.9, Lemma 10.1, and the condition $\sigma \sqrt{2 \log 1 / q} \leq \frac{1}{2 \sqrt{M_{2}}}$ which follows from our assumption on $\sigma$.

To compute the probability that the spacing is at least $\epsilon_{a}$, where $\epsilon_{a}>0$ will be chosen later, we condition on fixing of $A_{b}^{T} u=z$ and any fixing of $v$ :

$$
\operatorname{Pr}\left(\left|\frac{g_{a}\left(A_{a}^{T} u\right)}{g_{a}\left(A_{a}^{T} v\right)}-\frac{g_{b}\left(A_{b}^{T} u\right)}{g_{b}\left(A_{b}^{T} v\right)}\right| \leq \epsilon_{a}\right)=\int \operatorname{Pr}\left(\left|\frac{g_{a}\left(A_{a}^{T} u\right)}{g_{a}\left(A_{a}^{T} v\right)}-\frac{g_{b}(z)}{g_{b}\left(A_{b}^{T} v\right)}\right| \leq \epsilon_{a} \mid A_{b}^{T} u=z\right) \operatorname{Pr}\left(A_{b}^{T} u=z\right) d z .
$$

We will bound the conditional probability term. As in the proof of Theorem 6.3 applying Taylor's theorem with remainder (Theorem 4.8) gives

$$
g_{a}\left(A_{a}^{T} u\right)=i^{d} \sum_{l=d}^{k_{a}} \operatorname{cum}_{l}\left(s_{a}\right) \frac{\left(i\left(A_{a}^{T} u\right)\right)^{l-d}}{(l-d) !}+R_{k_{a}+1}\left(A_{a}^{T} u\right) \frac{\left(A_{a}^{T} u\right)^{k_{a}-d+1}}{\left(k_{a}-d+1\right) !} .
$$

Truncating $g_{a}$ after the degree $\left(k_{a}-d\right)$ term yields polynomial $p_{a}\left(A_{a}^{T} u\right)$. Denote the truncation error by $\rho_{a}\left(A_{a}^{T} u\right)$.

Then setting $h=\frac{g_{b}\left(A_{b}^{T} u\right) g_{a}\left(A_{a}^{T} v\right)}{g_{b}\left(A_{b}^{T} v\right)}$ which is a constant under our conditioning, we have

$$
\begin{aligned}
\left|\frac{g_{a}\left(A_{a}^{T} u\right)}{g_{a}\left(A_{a}^{T} v\right)}-\frac{g_{b}\left(A_{b}^{T} u\right)}{g_{b}\left(A_{b}^{T} v\right)}\right| & =\frac{1}{\left|g_{a}\left(A_{a}^{T} v\right)\right|}\left|g_{a}\left(A_{a}^{T} u\right)-\frac{g_{b}\left(A_{b}^{T} u\right) g_{a}\left(A_{a}^{T} v\right)}{g_{b}\left(A_{b}^{T} v\right)}\right| \\
& =\frac{1}{\left|g_{a}\left(A_{a}^{T} v\right)\right|}\left|g_{a}\left(A_{a}^{T} u\right)-h\right| \\
& =\frac{1}{\left|g_{a}\left(A_{a}^{T} v\right)\right|}\left|p_{a}\left(A_{a}^{T} u\right)+\rho_{a}\left(A_{a}^{T} u\right)-h\right| \\
& \geq \frac{1}{\left|g_{a}\left(A_{a}^{T} v\right)\right|}\left|p_{a}\left(A_{a}^{T} u\right)-h\right|-\frac{1}{\left|g_{a}\left(A_{a}^{T} v\right)\right|}\left|\rho_{a}\left(A_{a}^{T} u\right)\right| .
\end{aligned}
$$

Now we are going to show that the first term above is likely to be large and the second one is likely to be small.

We have $A_{a}^{T} u=\left\langle A_{a}, A_{b}\right\rangle A_{b}^{T} u+r^{T} u$ where $r$ is a vector orthogonal to $A_{b}$. Our hypothesis, namely $1-\left\langle A_{a}, A_{b}\right\rangle^{2} \geq L^{2}$, gives $\|r\|^{2} \geq L^{2}$. The orthogonality of $r$ and $A_{b}$ implies that the univariate Gaussian $r^{T} u$ is independent of $A_{b}^{T} u$. 
Now we apply our anti-concentration inequality from Theorem 4.4 to obtain (for $u \sim N\left(0, \sigma^{2} I_{n}\right.$ ) and fixed $v$ satisfying (28))

$$
\begin{aligned}
\operatorname{Pr}\left(\left|p_{a}\left(A_{a}^{T} u\right)-h\right| \leq \epsilon_{a} \mid A_{b}^{T} u=z\right) & \leq \frac{4\left(k_{a}-d\right)}{\sigma\|r\| \sqrt{2 \pi}}\left(\frac{\epsilon_{a}\left(k_{a}-d\right) !}{\left|\operatorname{cum}_{k_{a}}\left(s_{a}\right)\right|}\right)^{1 /\left(k_{a}-d\right)} \\
& \leq \frac{4\left(k_{a}-d\right)}{\sigma L \sqrt{2 \pi}}\left(\frac{\epsilon_{a}\left(k_{a}-d\right) !}{\Delta}\right)^{1 /\left(k_{a}-d\right)} .
\end{aligned}
$$

We choose

$$
\epsilon_{a}:=\frac{\Delta}{\left(k_{a}-d\right) !}\left(\frac{\sigma L q \sqrt{2 \pi}}{4\left(k_{a}-d\right)}\right)^{k_{a}-d} \geq \frac{\Delta}{(k-d) !}\left(\frac{\sigma L q \sqrt{2 \pi}}{4(k-d)}\right)^{k-d}=: \epsilon,
$$

making RHS of (31) equal to $q$. Recall that this was for fixed $v$ satisfying (28).

For the truncation error, assuming that the event $\left|A_{a}^{T} u\right| \leq \sigma \sqrt{2 \log 1 / q}$ happens, we have

$$
\begin{aligned}
\left|\rho_{a}\left(A_{a}^{T} u\right)\right| & \leq\left|\psi^{\left(k_{a}+1\right)}\left(A_{a}^{T} u\right)\right| \cdot \frac{\left(A_{a}^{T} u\right)^{k_{a}-d+1}}{\left(k_{a}-d+1\right) !} \\
& \leq \frac{2^{k_{a}} M_{k_{a}+1}}{(3 / 4)^{k_{a}+1}} \cdot \frac{k_{a} !}{\left(k_{a}-d+1\right) !} \cdot(\sigma \sqrt{2 \log 1 / q})^{k_{a}-d+1} \\
& \leq \epsilon_{a} / 2
\end{aligned}
$$

where to upper bound $\left|\psi^{\left(k_{a}+1\right)}\left(A_{a}^{T} u\right)\right|$ we used Lemma 4.9, Lemma 10.1, and the condition $\sigma \sqrt{2 \log (1 / q)} \leq$ $\frac{1}{2 \sqrt{M_{2}}}$, which holds given our upper bound on $\sigma$. And the final inequality follows from our condition $\sigma \leq \sigma^{\prime}$.

Thus with probability at least $1-\frac{2 q}{\sqrt{\pi \log 1 / q}}-q$ we have $\left|p_{a}\left(A_{a}^{T} u\right)-h\right|-\left|\rho_{a}\left(A_{a}^{T} u\right)\right| \geq \epsilon_{a} / 2$ under the condtion that $A_{b}^{T} u=z$ and $v$ fixed. Now since this holds for any $z$ and any fixing of $v$, it also holds without the conditioning on the event $A_{b}^{T} u=z$ and fixing of $v$.

Hence using (30), with probability at least $1-\frac{2 q}{\sqrt{\pi \log 1 / q}}-q \geq 1-3 q$ we have

$$
\frac{1}{\left|g_{a}\left(A_{a}^{T} v\right)\right|}\left(\left|p_{a}\left(A_{a}^{T} u\right)-h\right|-\left|\rho_{a}\left(A_{a}^{T} u\right)\right|\right) \geq \epsilon_{a} \cdot(3 / 8)^{d} \frac{1}{(d-1) ! M_{d}} \geq \epsilon \cdot(3 / 8)^{d} \frac{1}{(d-1) ! M_{d}} .
$$

To summarize, with probability at least $1-3 q$ the spacing is at least $\epsilon$. By the union bound, with probability at least $1-3\left(\begin{array}{c}m \\ 2\end{array}\right) q$ all the spacings are at least $\epsilon$.

The following is a straightforward corollary of the proof of the previous theorem.

Corollary 6.4. In the setting of Theorem 6.3 we have with probability at least $1-6 m q$ that

$$
\left|g_{a}\left(A^{T} u\right)\right|,\left|g_{a}\left(A^{T} v\right)\right| \geq \frac{\Delta_{0}}{2(k-d) !}\left(\frac{\sigma q \sqrt{2 \pi}}{4(k-d)}\right)^{k-d}
$$

for all $a \in[m]$.

An important part of the proof is to give a lower bound on the quantity $1-\left\langle A_{i}, A_{j}\right\rangle^{2} \geq L^{2}$ so that the ICA model remains identifiable. At order $d$, we will give our bounds in terms of $\sigma_{m}\left(A^{\odot j}\right)$ for $j=1, \ldots, d / 2$.

Lemma 6.5. Fix $m, n \in \mathbb{N}$ such that $n \leq m$. Let $A \in \mathbb{C}^{n \times m}$ be a full row rank matrix whose columns $A_{i}$ have unit norm. Then

$$
1-\left\langle A_{i}, A_{j}\right\rangle^{2} \geq \frac{2}{k} \sigma_{m}\left(A^{\odot k}\right)^{2}
$$

for all $k \in \mathbb{N}$ where $k \geq 2$. 
Proof. Consider the matrix $B=A^{\odot 2}$, observe that $\left\langle A_{i}, A_{j}\right\rangle^{2}=\left\langle B_{i}, B_{j}\right\rangle$. Define the matrix $C=A^{\odot k}$. Observe that $\left\|C_{i}\right\|=\left\|A_{i}\right\|^{k}=1$ and that

$$
1-\left\langle B_{i}, B_{j}\right\rangle=1-\left|\left\langle C_{i}, C_{j}\right\rangle\right|^{2 / k}
$$

for $k \geq 2$. Recall that

$$
\sigma_{m}(C)=\min _{\|x\|=1}\|C x\|
$$

In particular, if we consider the vector $x=\frac{1}{\sqrt{2}}\left(e_{i} \pm e_{j}\right)$ we have

$$
\|C x\|^{2}=\frac{1}{2}\left(\left\|C_{i}\right\|^{2}+\left\|C_{j}\right\|^{2} \pm 2\left\langle C_{i}, C_{j}\right\rangle\right)=1 \pm\left\langle C_{i}, C_{j}\right\rangle \geq \sigma_{m}(C)^{2} .
$$

Hence we must have $1-\left|\left\langle C_{i}, C_{j}\right\rangle\right| \geq \sigma_{m}^{2}(C)$. Combining this with (32), we obtain

$$
\begin{aligned}
1-\left\langle B_{i}, B_{j}\right\rangle & =1-\left|\left\langle C_{i}, C_{j}\right\rangle\right|^{2 / k} \\
& \geq 1-\left(1-\sigma_{m}(C)^{2}\right)^{2 / k} \\
& \geq \frac{2}{k} \sigma_{m}(C)^{2},
\end{aligned}
$$

where the last step follows from noting that all derivatives of the function $f(x)=(1-x)^{t}$ for $t \in(0,1)$ are negative in the interval $x \in[0,1]$

\subsection{Sample complexity}

To understand the complexity of our algorithm, we must bound how many samples are needed to estimate the matrices $M_{u}$ and $M_{v}$ accurately. Throughout this section, we estimate $\mathbb{E}(f(x))$ for some function $f(x)$, using independent samples $x^{i}$ via

$$
\bar{E}(f(x)):=\frac{1}{N} \sum_{i=1}^{N} f\left(x^{i}\right) \rightarrow \mathbb{E}(f(x)) .
$$

More generally, we will estimate derivative tensors as follows. As before, $\phi(t)=\mathbb{E}\left(e^{i t^{T} s}\right)$ and define the empirical version of the characteristic function in the natural way $\bar{\phi}(t):=\frac{1}{N} \sum_{i=1}^{N} e^{i t^{T} s^{i}}$. As we will see, for a multiset $S \subseteq[m]$ the derivative of $\bar{\phi}(t)$ behaves nicely and will serve as an approximation of $\phi(t)$. Note that

$$
\partial_{S} \bar{\phi}(t)=\overline{\mathbb{E}}\left(s_{S} e^{i t^{T} s}\right)
$$

where $\overline{\mathbb{E}}(\cdot)$ denotes empirical expectation over $N$ i.i.d. samples of $s$. Similarly, we estimate $\partial_{S} \log \phi(t)$ by

$$
\partial_{S} \log \bar{\phi}(t)=\bar{N}_{d}(t) / \bar{\phi}(t)^{d},
$$

where by Claim $6.2 \bar{N}_{d}(t)$ is a sum of the form $\sum_{S_{1}, \ldots, S_{d}} C_{S_{1}, \ldots, S_{d}}\left(\partial_{S_{1}} \bar{\phi}(t)\right) \ldots\left(\partial_{S_{d}} \bar{\phi}(t)\right)$, as described in Claim 6.2. Thus to show that $\partial_{S} \bar{\phi}(t)$ is a good approximation of $\partial_{S} \phi(t)$ we show that $\left|\frac{N_{d}(t)}{\phi(t)^{d}}-\frac{\bar{N}_{d}(t)}{\phi(t)^{d}}\right|=$ $\frac{\left|\bar{\phi}(t)^{d} N_{d}(t)-\phi(t) \bar{N}_{d}(t)\right|}{\phi(t)^{d} \phi(t)^{d}}$ is small.

The notion of empirical estimate of a derivative tensor now follows immediately from (33) which gives how to estimate individual entries of the tensor. 
Lemma 6.6. Let $s \in \mathbb{R}^{m}$ be a random vector with independent components. For $t \in \mathbb{R}^{m}$ let $\psi_{s}(t)=\phi_{s}(t)=$ $\log \mathbb{E}\left(e^{i t^{t} s}\right)$ be the second characteristic function of $s$. Consider the dth derivative tensor of $\psi_{s}(t)$ (it contains $m^{d}$ entries). Let $M_{2}, M_{2 d}>0$ be such that $\mathbb{E}\left(s_{i}^{2}\right) \leq M_{2}$ and $\mathbb{E}\left(\left|s_{i}\right|^{2 d}\right) \leq M_{2 d}$. Fix $0<\epsilon, \delta<1 / 4$, and let $\|t\| \leq \frac{1}{\sqrt{2 M_{2}}}$. Suppose we take $N$ samples then with probability at least

$$
1-\left(\begin{array}{c}
m+d-1 \\
d
\end{array}\right) \frac{2^{d} M_{2 d}}{\epsilon^{2} \delta}\left[\frac{2^{d} d\left(M_{d}+2\right)^{d-1}(d-1) !}{(3 / 4)^{d}(1 / 2)^{d}}\right]^{2}
$$

every entry of the empirical tensor will be within $\epsilon$ of the corresponding entry of the derivative tensor.

Proof. In light of (33) we will prove that each term in the expression for $\bar{N}_{d}(t)$ (it's a product of several $\left.\partial_{S} \bar{\phi}(t)\right)$ is a good approximation of the corresponding term in the expression for $N_{d}(t)$ by showing that the corresponding factors in the product are close. Finally, we show that the whole sum is a good approximation. For complex-valued r.v. $X$ with mean $\mu$, note that $\operatorname{Var}(X)=\mathbb{E}\left((X-\mu)(X-\mu)^{*}\right)=\mathbb{E}\left(X X^{*}\right)-\mu \mu^{*} \leq$ $\mathbb{E}\left(|X|^{2}\right)$.

We use the second moment method to prove that $\partial_{S} \bar{\phi}(t)$ is a good approximation of $\partial_{S} \phi(t)$ with high probability.

For multiset $S \subseteq\left\{i_{1}, \ldots, i_{d}\right\}$, with $p_{j}$ being the frequency of $j$ in $S$, by the same arguments as in (26) we have

$$
\operatorname{Var}\left(s_{S} e^{i t^{T} s}\right) \leq \mathbb{E}\left(s_{S}^{2}\right) \leq \prod_{j=1}^{m} \mathbb{E}\left(s_{j}^{2 p_{j}}\right) \leq \prod_{j=1}^{m}\left(\mathbb{E}\left(s_{j}^{2 d}\right)\right)^{p_{j} / d} \leq\left(\max _{j} \mathbb{E}\left(s_{j}^{2 d}\right)\right)^{|S| / d} \leq M_{2 d}^{|S| / d}
$$

Thus

$$
\operatorname{Var}\left(\partial_{S} \bar{\phi}(t)\right) \leq \frac{M_{2 d}^{|S| / d}}{N} \leq \frac{M_{2 d}}{N}
$$

Chebyshev's inequality (which remains unchanged for complex-valued r.v.s) for $\epsilon^{\prime}>0$ yields

$$
\operatorname{Pr}\left(\left|\partial_{S} \bar{\phi}(t)-\partial_{S} \phi(t)\right| \geq \epsilon^{\prime}\right) \leq \frac{M_{2 d}}{\epsilon^{\prime 2} N}
$$

We will choose a value of $\epsilon^{\prime}$ shortly.

Now we bound the difference between the corresponding summands in the decompositions of $N_{d}(t)$ and $\bar{N}_{d}(t)$ as sums. Specifically, with probability at most $\frac{(d+1) M_{2 d}}{\epsilon^{\prime} N}$ (this comes from the union bound: we want the event in (34) to hold for all $S_{j}$ for $j \in[d]$ as well as for $S=\emptyset$, corresponding to $\left.\phi(t)\right)$ we have

$$
\begin{aligned}
\left|\left(\bar{\phi}(t)^{d} \prod_{j=1}^{d} \partial_{S_{j}} \phi(t)\right)-\left(\phi(t)^{d} \prod_{j=1}^{d} \partial_{S_{j}} \bar{\phi}(t)\right)\right| & \leq\left|\prod_{j=1}^{d} \partial_{S_{j}} \phi(t)\right|\left|\bar{\phi}(t)^{d}-\phi(t)^{d}\right|+\left|\phi(t)^{d}\right|\left|\prod_{j=1}^{d} \partial_{S_{j}} \phi(t)-\prod_{j=1}^{d} \partial_{S_{j}} \bar{\phi}(t)\right| \\
& \leq M_{d}\left|\bar{\phi}(t)^{d}-\phi(t)^{d}\right|+\left(M_{d}+\epsilon^{\prime}\right)^{d}-M_{d}^{d} \\
& \leq \epsilon^{\prime} d M_{d}+\epsilon^{\prime} d\left(M_{d}+\epsilon^{\prime}\right)^{d-1} \\
& \leq 2 \epsilon^{\prime} d\left(M_{d}+1+\epsilon^{\prime}\right)^{d-1}
\end{aligned}
$$

where the second inequality used (26), Lemma 10.2 and $|\phi(t)| \leq 1$ and $|\bar{\phi}(t)| \leq 1$.

Now using the expression for $N_{d}(t)$ as a sum given in Claim 6.2, with probability at most $\frac{2^{d} M_{2 d}}{\epsilon^{\prime 2} N}($ the factor $2^{d}$ again comes from the union bound: we want the event in (34) to hold for all (multi-) subsets of 
$\left.\left\{i_{1}, \ldots, i_{d}\right\}\right)$ we have

$$
\begin{aligned}
\left|\partial_{S} \bar{\phi}(t)-\partial_{S} \phi(t)\right| & =\frac{\left|\bar{\phi}(t)^{d} N_{d}(t)-\phi(t) \bar{N}_{d}(t)\right|}{\left|\phi(t)^{d} \bar{\phi}(t)^{d}\right|} \\
& \leq \frac{2^{d} \epsilon^{\prime} d\left(M_{d}+1+\epsilon^{\prime}\right)^{d-1}(d-1) !}{\left|\phi(t)^{d} \bar{\phi}()^{d}\right|} \\
& \leq \frac{2^{d} \epsilon^{\prime} d\left(M_{d}+1+\epsilon^{\prime}\right)^{d-1}(d-1) !}{(3 / 4)^{d}\left(3 / 4-\epsilon^{\prime}\right)^{d}} \\
& \leq \epsilon
\end{aligned}
$$

where the last inequality used Lemma 10.1 and

$$
\epsilon^{\prime}=\left[\frac{2^{d} d\left(M_{d}+1+\epsilon^{\prime}\right)^{d-1}(d-1) !}{(3 / 4)^{d}\left(3 / 4-\epsilon^{\prime}\right)^{d}}\right]^{-1} \epsilon .
$$

Now if we want (36) to hold for all multisets $S$ of size $d$, then the union bound needs to extended to all such multisets (of which there are $\left(\begin{array}{c}m+d-1 \\ d\end{array}\right)$ ) giving that error probability at most

$$
\left(\begin{array}{c}
m+d-1 \\
d
\end{array}\right) \frac{2^{d} M_{2 d}}{\epsilon^{\prime 2} N}=\left(\begin{array}{c}
m+d-1 \\
d
\end{array}\right) \frac{2^{d} M_{2 d}}{\epsilon^{2} N}\left[\frac{2^{d} d\left(M_{d}+1+\epsilon^{\prime}\right)^{d-1}(d-1) !}{(3 / 4)^{d}\left(3 / 4-\epsilon^{\prime}\right)^{d}}\right]^{2},
$$

as desired.

Lemma 6.7 (Sample Complexity). Let $x=$ As be an ICA model with $A \in \mathbb{R}^{n \times m}, x \in \mathbb{R}^{n}, s \in \mathbb{R}^{m}$ and $d$ an even positive integer. Let $M_{2}, M_{2 d}>0$ be such that $\mathbb{E}\left(s_{i}^{2}\right) \leq M_{2}$ and $\mathbb{E}\left(\left|s_{i}\right|^{2 d}\right) \leq M_{2 d}$. Let $v \in \mathbb{R}^{n}$ satisfy $\|v\|_{2} \leq \frac{1}{2\|A\|_{2} \sqrt{2 M_{2}}}$. Let $T_{v}=D_{u}^{d} \psi_{x}(v)$ be the d'th derivative tensor of $\psi_{x}(u)=\log \mathbb{E}\left(e^{i u^{T} x}\right)$ at $v$. And let $\bar{T}_{v}=D_{u}^{d} \bar{\psi}_{x}(v)$ be its naive estimate using $N$ independent samples of $x$ where

$$
N \geq\left(\begin{array}{c}
m+d-1 \\
d
\end{array}\right) \frac{1}{m^{d / 2} \sigma_{1}(A)^{d}} \frac{2^{d} M_{2 d}}{\epsilon^{2} \delta}\left[\frac{2^{d} d\left(M_{d}+2\right)^{d-1}(d-1) !}{(3 / 4)^{d}(1 / 2)^{d}}\right]^{2} .
$$

Then with probability at least $1-\delta$ we have

$$
\left\|T_{v}-\bar{T}_{v}\right\|_{F} \leq \epsilon
$$

Proof. In the following all tensors are flattened into matrices. Let $x^{j}=A s^{j}, j \in[N]$ be i.i.d. samples. Letting $t=A^{T} v$ we have $T_{v}=D_{u}^{d} \psi_{x}(u)=A^{\otimes d / 2} D_{t}^{d} \psi_{s}(t)\left(A^{\otimes d / 2}\right)^{T}$, and $\bar{T}_{v}=D_{u}^{d} \bar{\psi}_{x}(u)=A^{\otimes d / 2} D_{t}^{d} \bar{\psi}_{s}(t)\left(A^{\otimes d / 2}\right)^{T}$. (Note that we could also have written $D_{u}^{d} \psi_{x}(u)=A^{\odot d / 2} \operatorname{diag}\left(\partial_{t_{j}}^{d} \psi_{s}(t)\right)\left(A^{\odot d / 2}\right)^{T}$ because the components of $s$ are indpendent, however the corresponding empirical equation $D_{u}^{d} \bar{\psi}_{x}(u)=A^{\odot d / 2} \operatorname{diag}\left(\partial_{t_{j}}^{d} \bar{\psi}_{s}(t)\right)\left(A^{\odot d / 2}\right)^{T}$ need not be true.)

Hence

$$
\begin{aligned}
\left\|\bar{T}_{v}-T_{v}\right\|_{F} & =\left\|A^{\otimes d / 2} D_{t}^{d} \bar{\psi}_{s}(t)\left(A^{\otimes d / 2}\right)^{T}-A^{\otimes d / 2} D_{t}^{d} \psi_{s}(t)\left(A^{\otimes d / 2}\right)^{T}\right\|_{F} \\
& =\left\|A^{\otimes d / 2}\left(D_{t}^{d} \bar{\psi}_{s}(t)-D_{t}^{d} \psi_{s}(t)\right)\left(A^{\otimes d / 2}\right)^{T}\right\|_{F} \\
& \leq \sigma_{1}\left(A^{\otimes d / 2}\right)^{2}\left\|D_{t}^{d} \bar{\psi}_{s}(t)-D_{t}^{d} \psi_{s}(t)\right\|_{F} \\
& =\sigma_{1}(A)^{d}\left\|D_{t}^{d} \bar{\psi}_{s}(t)-D_{t}^{d} \psi_{s}(t)\right\|_{F} \\
& \leq \epsilon
\end{aligned}
$$

where the last inequality holds with probability at least $1-\delta$ by Lemma 6.6 which is applicable because $\left\|A^{T} v\right\|_{2} \leq\|A\|_{2}\|v\|_{2} \leq \frac{1}{2 \sqrt{2 M_{2}}}$. 


\subsection{Main theorem and proof}

We are now ready to formally state and prove the main theorem. To get a success probability of $3 / 4$, we choose $q$ so that $20 m^{2} q<1 / 4$.

Theorem 6.8 (Underdetermined ICA). Let $x \in \mathbb{R}^{n}$ be generated by an underdetermined ICA model $x=$ As with $A \in \mathbb{R}^{n \times m}$ where $n \leq m$. Suppose that the following data and conditions are given:

1. $d \in 2 \mathbb{N}$ such that $\sigma_{m}(A \odot d / 2)>0$.

2. $k$ such that for each $i$ there exists $k_{i}$, where $d<k_{i}<k$ such that $\left|\operatorname{cum}_{k_{i}}\left(s_{i}\right)\right| \geq \Delta_{0}$.

3. Constants $M_{2}, M_{d}, M_{k}$ such that for each $s_{i}$ the following bounds hold

$$
\begin{gathered}
\mathbb{E}\left(s_{i}\right)=0, \quad \mathbb{E}\left(s_{i}^{2}\right) \leq M_{2}, \quad \mathbb{E}\left(s_{i}^{d}\right) \leq M_{d}, \quad \mathbb{E}\left(\left|s_{i}\right|^{k_{i}+1}\right) \leq M_{k}, \quad \Delta_{0} \leq M_{d}, \quad \mathbb{E}\left(\left|s_{i}\right|^{2 d}\right) \leq M_{2 d} \\
\text { 4. } 0<\sigma \leq \min \left(1, \sigma_{0}, \frac{1}{4 m} \sqrt{\frac{1}{6 M_{2} \ln (2 / q)}}\right) \text { where } \\
\sigma_{0}=\Delta_{0} \frac{k-d+1}{k !}\left(\frac{3}{8}\right)^{k} \frac{1}{M_{k}}\left(\frac{2 \sigma_{m}\left(A^{\odot d / 2}\right) q \sqrt{2 \pi}}{4(k-d) \sqrt{d}}\right)^{k-d}\left(\frac{1}{\sqrt{2 \log 1 / q}}\right)^{k-d} \cdot
\end{gathered}
$$

Then, with probability at least $1-20 m^{2} q$, algorithm Underdetermined $\boldsymbol{I C A}(\sigma)$ will return a matrix $\tilde{B}$ such that there exist signs $\alpha_{j} \in\{-1,1\}$ and permutation $\pi:[m] \rightarrow[m]$ such that

$$
\left\|B_{j}-\alpha_{j} \tilde{B}_{\pi(j)}\right\| \leq \epsilon
$$

using $N$ samples where

$$
N \geq\left(\frac{k m\left(M_{d}+2\right)}{\sigma q \sigma_{m}\left(A^{\odot d / 2}\right)}\right)^{c k} \frac{\kappa\left(A^{\odot d / 2}\right)^{6} M_{2 d}}{\Delta_{0}^{6} \epsilon^{2}},
$$

for some absolute constant c. The running time of the algorithm is poly $(N)$.

Proof. The proof involves putting together of various results we have proven. We take $N$ independent samples of $x$ and form the flattened $d$ th derivative tensors $\bar{M}_{u}, \bar{M}_{v}$ of $\psi(u)$ evaluated at $u$ and $v$ which are sampled from $N\left(0, \sigma_{0}^{2}\right)$. Recall that these are the matrices constructed by Underdetermined $\left.\mathbf{I C A}\left(\sigma_{0}\right)\right)$ which then invokes Diagonalize(.) which computes eigendecomposition of $\bar{M}_{u} \bar{M}_{v}^{-1}$. We will denote by $M_{u}, M_{v}$ the corresponding matrices without any sampling errors. We will first use the result about eigenvalue spacing Theorem 6.3 to get a bound on the spacings of the eigenvalues of the matrix $M_{u} M_{v}^{-1}$, where $u, v \sim N\left(0, \sigma_{0}^{2} I_{n}\right)$ are random vectors. Next, we determine upper and lower bounds $K_{U}$ and $K_{L}$ on the eigenvalues of $M_{u}$ and $M_{v}$. We can then apply Theorem 5.4 to show that if we have sufficiently good approximation of $M_{u}$ and $M_{v}$ then we will get a good reconstruction of matrix $A$. Finally, we use Lemma 6.7 to determine the number of samples needed to get the required approximation.

Step 1. First, we apply Theorem 6.3. Note that our choice of $\sigma_{0}$ satisfies the constraints on $\sigma$ in Theorem 6.3 thus except with probability $q$, we have

$$
\left|\frac{g_{b}\left(A_{b}^{T} u\right)}{g_{b}\left(A_{b}^{T} v\right)}-\frac{g_{a}\left(A_{a}^{T} u\right)}{g_{a}\left(A_{a}^{T} v\right)}\right| \geq \Omega_{0}:=\frac{\Delta_{0}}{M_{d}}\left(\frac{3}{8}\right)^{d} \frac{1}{(d-1) !(k-d) !}\left(\frac{\sigma_{0} q L \sqrt{2 \pi}}{4(k-d)}\right)^{k-d}
$$

for all pairs $a, b \in[m]$. Here $L$ as defined in Theorem 6.3 is given by $2 \sigma_{m}\left(A^{\odot} d / 2\right) / \sqrt{d}$ by Lemma 6.5 
Step 2. Next, we will show that $u$ and $v$ concentrate in norm. To do so, we will apply the following concentration inequality for sub-exponential random variables (this is standard in the proof of the JohnsonLindenstrauss Lemma, see [10, 29] or alternatively [66] for a more general formulation).

Lemma 6.9 ([10, 29]). Let $z_{i} \sim N(0,1)$ be i.i.d., then

$$
\operatorname{Pr}\left(\sum_{i=1}^{n} z_{i}^{2} \geq \beta n\right) \leq e^{\frac{n}{2}(1-\beta+\log (\beta))} .
$$

For $\beta \geq 6$, the bound only improves as $n$ increases. Thus, we have the simplified bound

$$
\operatorname{Pr}\left(\sum_{i=1}^{n} z_{i}^{2} \geq \beta n\right) \leq e^{-\frac{n \beta}{12}} .
$$

In particular, union bounding over both $u, v \in \mathbb{R}^{n}$, we have

$$
\begin{aligned}
\operatorname{Pr}\left(\|u\|,\|v\| \geq \frac{1}{2\|A\|_{F} \sqrt{2 M_{2}}}\right) & =\operatorname{Pr}\left(\|u\|^{2},\|v\|^{2} \geq\left(\frac{1}{2\|A\|_{F} \sqrt{2 M_{2}}}\right)^{2}\right) \\
& \leq 2 \exp \left(-\frac{1}{12 \sigma_{0}^{2}}\left(\frac{1}{2\|A\|_{F} \sqrt{2 M_{2}}}\right)^{2}\right)
\end{aligned}
$$

where in the second line, we took $\beta n=\frac{1}{12 \sigma_{0}^{2}}\left(\frac{1}{2\|A\|_{F} \sqrt{2 M_{2}}}\right)^{2}$. Using $\|A\|_{F} \leq m$, and our choice of $\sigma_{0}$ which gives $\sigma_{0} \leq \frac{1}{4 m} \sqrt{\frac{1}{6 M_{2} \ln (2 / q)}}$ we obtain

$$
\operatorname{Pr}\left(\|u\|,\|v\| \geq \frac{1}{2\|A\|_{F} \sqrt{2 M_{2}}}\right) \leq q .
$$

Thus except with probability $q$, norms $\|u\|,\|v\|$ satisfy the hypotheses of Lemma 6.7.

Step 3. Now we determine the values of parameters $K_{U}$ and $K_{L}$ used in Theorem 5.4 A bound for $K_{U}$ can be obtained from Lemma 4.9 and Lemma 10.1 to $\psi_{s}(t)=\psi_{s}\left(A^{T} u\right)$. The latter lemma being applicable because $\left\|A^{T} u\right\| \leq\|A\|_{F}\|u\| \leq \frac{1}{2 \sqrt{2 M_{2}}}$ and $\left\|A^{T} v\right\| \leq\|A\|_{F}\|v\| \leq \frac{1}{2 \sqrt{2 M_{2}}}$ from Step 2:

$$
K_{U}=\frac{(d-1) ! 2^{d-1} M_{d}}{(3 / 4)^{d}}
$$

For $K_{L}$, by Cor. 6.4 we can set

$$
K_{L}=\frac{\Delta_{0}}{2(k-d) !}\left(\frac{\sigma_{0} q \sqrt{2 \pi}}{4(k-d)}\right)^{k-d},
$$

which holds with probability at least $1-6 m q$.

Step 4. We now fix $K_{1}$ which is the upper bound on $\left\|M_{u}-\bar{M}_{u}\right\|_{F}$ and $\left\|M_{v}-\bar{M}_{v}\right\|_{F}$ needed in Theorem5.4 (the role of these two quantities is played by $\left\|R_{\mu}\right\|_{F}$ and $\left\|R_{\lambda}\right\|_{F}$ in that theorem). Our assumption $\Delta_{0} \leq M_{d}$ gives that $\Omega_{0} \leq 1$ by (37). And hence the bound required in Theorem 5.4 becomes

$$
K_{1}=\frac{\epsilon K_{L}^{2} \sigma_{m}(B)^{3}}{2^{11} \kappa(B)^{3} K_{U} m^{2}} \Omega_{0},
$$


where $B=A^{\odot d / 2}$.

For this $K_{1}$ by Theorem 5.4 the algorithm recovers $\tilde{B}$ with the property that there are signs $\alpha_{j} \in\{-1,1\}$ and permutation $[m] \rightarrow[m]$ such that

$$
\left\|B_{j}-\alpha_{j} \tilde{B}_{\pi(j)}\right\| \leq \epsilon
$$

Step 5. It remains to determine the number of samples needed to achieve $\left\|M_{u}-\bar{M}_{u}\right\|_{F} \leq K_{1}$ and $\left\|M_{v}-\bar{M}_{v}\right\|_{F} \leq K_{1}$.

By Step 2 above, we satisfy the hypotheses of Lemma 6.7. Hence by that lemma, for $N$ at least the quantity below

$$
\left(\begin{array}{c}
m+d-1 \\
d
\end{array}\right) \frac{1}{m^{d / 2} \sigma_{1}(A)^{d}} \frac{2^{d} M_{2 d}}{K_{1}^{2} q}\left(\frac{16^{d} d\left(M_{d}+2\right)^{d-1}(d-1) !}{3^{d}}\right)^{2} \leq 11^{2 d} m^{d / 2} d^{2(d+1)} M_{2 d}\left(M_{d}+2\right)^{2(d-1)} \frac{1}{K_{1}^{2} q}
$$

we have

$$
\left\|M_{u}-\bar{M}_{u}\right\|_{F} \leq K_{1},
$$

except with probability $q$, and similarly for $\left\|M_{u}-\bar{M}_{u}\right\|_{F}$. Subtistuting the value of $K_{1}$ from (38) and in turn of $K_{U}, K_{L}$ and $\Omega_{0}$ above and simplifying (we omit the straightforward but tedious details) gives that it suffices to take

$$
N \geq \frac{2^{4 k+6 d+26}}{3^{2 d}} d^{6 d+2}(k-d)^{2(k-d)} m^{d / 2+4} \frac{M_{d}^{2} M_{2 d}\left(M_{d}+2\right)^{2 d}}{\Delta_{0}^{6}} \frac{\kappa(B)^{6}}{\sigma_{m}(B)^{k-d+6}} \frac{1}{\sigma^{5(k-d)} q^{5(k-d)+1}} \frac{1}{\epsilon^{2}} .
$$

Accounting for the probability of all possible bad events enumerated in the proof via the union bound we see that with probability at least $1-q-3\left(\begin{array}{c}m \\ 2\end{array}\right) q-6 m q-q>1-20 m^{2} q$ no bad events happen. The running time computation involves empirical estimates of derivate tensors and SVD and eigenvalue computations; we skip the routine check that the running time is $\operatorname{poly}(N)$.

\subsection{Underdetermined ICA with unknown Gaussian noise}

Theorem 6.8 just proved is the detailed version of Theorem 1.3 without Gaussian noise. In this section we indicate how to extend this proof when there is Gaussian noise thus proving Theorem 1.3 in full. Our algorithm for the noiseless case applies essentially unaltered to the case when the input has unknown Gaussian noise if $d>2$. We comment on the case $d=2$ at the end of this section. More precisely, the ICA model now is

$$
x^{\prime}=x+\eta=A s+\eta,
$$

where $\eta \sim N(0, \Sigma)$ where $\sigma \in \mathbb{R}^{n \times n}$ is unknown covariance matrix and $\eta$ is independent of $s$. Using the independence of $\eta$ and $s$ and the standard expression for the second characteristic of the Gaussian we have

$$
\psi_{x^{\prime}}(u)=\mathbb{E}\left(e^{i u^{T} x^{\prime}}\right)=\mathbb{E}\left(e^{i u^{T} x+i u^{T} \eta}\right)=\psi_{x}(u)+\psi_{\eta}(u)=\psi_{x}(u)-\frac{1}{2} u^{T} \Sigma u .
$$

Our algorithm works with (estimate of) the $d$ th derivative tensor of $\psi_{x^{\prime}}(u)$. For $d>2$, we have $D_{u}^{d} \psi_{x^{\prime}}(u)=D_{u}^{d} \psi_{x}(u)$ as in (39) the component of the second characteristic function corresponding to the Guassian noise is quadratic and vanishes for third and higher derivatives. Therefore, but for the estimation errors, the Gaussian noise makes no difference and the algorithm would still recover $A$ as before. Since the algorithm works only with estimates of these derivatives, we have to account for how much our estimate of $D_{u}^{d} \psi_{x}(u)$ changes due to the extra additive term involving the derivative of the estimate of the second characteristic of the Guassian. 
If $\Sigma$ is such that the moments of the Gaussian noise also satisfy the conditions we imposed on the moments of the $s_{i}$ in the Theorem 6.8 then we can complete the proof with little extra work. The only thing that changes in the proof of the main theorem is that instead of getting the bound $\left\|M_{u}-\bar{M}_{u}\right\| \leq \epsilon^{\prime}$ we get the bound $\left\|M_{u}-\bar{M}_{u}\right\| \leq 2 \epsilon^{\prime}$. If we increase the number of samples by a factor of 4 then this bound becomes $\left\|M_{u}-\bar{M}_{u}\right\| \leq \epsilon^{\prime}$, and so the proof can be completed without any other change.

The $d=2$ case. When $d=2$, the second derivative of the component of the second characteristic function corresponding to the noise in (39) is a constant matrix independent of $u$. Thus if we take derivatives at two different points and subtract them, then this constant matrix disappears. This is analogous to the algorithm we gave for fully-determined ICA with noise in Sec. 4.8. The error analysis can still proceed along the above lines; we omit the details.

\section{Mixtures of spherical Gaussians}

Here we apply Fourier PCA to the classical problem of learning a mixture of Gaussians, assuming each Gaussian is spherical. More precisely, we get samples $x+\eta$, where $x$ is from a distribution that is a mixture of $k$ unknown Gaussians, with $i$ 'th component having mixing weight $w_{i}$ and distribution $F_{i}=N\left(\mu_{i}, \sigma_{i}^{2} I\right)$; the noise $\eta$ is drawn from $N\left(\mu_{\eta}, \Sigma_{\eta}\right)$ and is not necessarily spherical. The problem is to estimate the unknown parameters $w_{i}, \mu_{i}, \sigma_{i}$. Our method parallels the Fourier PCA approach to ICA, but here, because the structure of the problem is additive (rather than multiplicative as in ICA), we can directly use the matrix $D^{2} \phi$ rather than $D^{2} \psi=D^{2} \log (\phi)$. It is easy to show that $D^{2} \phi=\Sigma_{u}$ in the description of our algorithm.

For any integrable function $f: \mathbb{C}^{n} \rightarrow \mathbb{C}$, we observe that for a mixture $F=\sum_{i=1}^{k} w_{i} F_{i}$ :

$$
\mathbb{E}_{F}((f(x+\eta)))=\sum_{i=1}^{k} w_{i} \mathbb{E}_{F_{i}}(f(x+\eta)) .
$$

We assume, without loss of generality, that the full mixture is centered at zero, so that:

$$
\sum_{i=1}^{k} w_{i} \mu_{i}=0
$$




\section{Fourier PCA for Mixtures}

1. Pick $u$ independently from $N\left(0, I_{n}\right)$.

2. Compute $M=\mathbb{E}\left(x x^{T}\right)$, let $V$ be the span of its top $k-1$ eigenvectors and $\bar{\sigma}^{2}$ be its $k$ 'th eigenvalue and $v$ be its $k^{\prime}$ th eigenvector. Let $z$ be a vector orthogonal to $V$ and to $u$.

3. Compute

$$
\begin{aligned}
& \Sigma_{u}=\mathbb{E}\left(x x^{T} e^{i u^{T} x}\right), \quad \bar{\sigma}_{u}^{2}=\mathbb{E}\left(\left(z^{T} x\right)^{2} e^{i u^{T} x}\right), \\
& \gamma_{u}=\frac{1}{\left(u^{T} v\right)^{2}}\left(-\mathbb{E}\left(\left(v^{T} x\right)^{2} e^{i u^{T} x}\right)+\bar{\sigma}_{u}^{2}\right), \quad \tilde{u}=\mathbb{E}\left(x\left(z^{T} x\right)^{2} e^{i u^{T} x}\right) .
\end{aligned}
$$

4. Compute the matrices

$$
M=\mathbb{E}\left(x x^{T}\right)-\sigma^{2} I \text { and } M_{u}=\Sigma_{u}-\tilde{\sigma}_{u}^{2} I-i \tilde{u} u^{T}-i u \tilde{u}^{T}-\gamma_{u} u u^{T} .
$$

5. Run Tensor Decomposition $\left(M_{u}, M\right)$ to obtain eigenvectors $\tilde{\mu}_{j}$ and eigenvalues $\lambda_{j}$ of $M_{u} M^{-1}$ (in their original coordinate representation).

6. Estimate mixing weights by finding $w \geq 0$ that minimizes $\left\|\sum_{j=1}^{k} \sqrt{w_{j}} \tilde{\mu}_{j}\right\|$ s.t. $\sum_{j=1}^{k} w_{j}=1$. Then estimate means and variances as

$$
\mu_{j}=\frac{1}{\sqrt{w_{j}}} \tilde{\mu}_{j}, \quad e^{-\frac{1}{2} \sigma_{j}^{2}\|u\|^{2}+i u^{T} \mu_{j}}=\lambda_{j}
$$

Lemma 7.1. For any $f: \mathbb{C}^{n} \rightarrow \mathbb{C}$, and $x \sim N(\mu, \Sigma)$ where $\Sigma$ is positive definite:

$$
\mathbb{E}\left(f(x) e^{i u^{T} x}\right)=e^{i u^{T} \mu-\frac{1}{2} u^{T} \Sigma u} \mathbb{E}(f(x+i \Sigma u)) .
$$

Proof. The proof is via a standard completing the square argument; consider the exponent:

$$
\begin{aligned}
- & \frac{1}{2}\left[(x-\mu)^{T} \Sigma^{-1}(x-\mu)\right]+i u^{T} x \\
=- & \frac{1}{2}\left[x^{T} \Sigma^{-1} x+\mu^{T} \Sigma^{-1} \mu-x^{T} \Sigma^{-1} \mu-\mu^{T} \Sigma^{-1} x\right]+i u^{T} x \\
=- & \frac{1}{2}\left[x^{T} \Sigma^{-1} x-x^{T} \Sigma^{-1}(\mu+i \Sigma u)-(\mu+i \Sigma u)^{T} \Sigma^{-1} x+(\mu+i \Sigma u)^{T} \Sigma^{-1}(\mu+i \Sigma u)\right] \\
& +i u^{T} \mu+\frac{1}{2}(\Sigma u)^{T} \Sigma^{-1}(\Sigma u) \\
=- & \frac{1}{2}(x-(\mu+i \Sigma u))^{T} \Sigma^{-1}(x-(\mu+i \Sigma u))+i u^{T} \mu-\frac{1}{2} u^{T} \Sigma u
\end{aligned}
$$

Thus:

$$
\begin{aligned}
& \mathbb{E}\left(f(x) e^{i u^{T} x}\right) \\
& =\frac{1}{\operatorname{det}(\Sigma)^{1 / 2}(2 \pi)^{n / 2}} \int f(x) e^{i u^{T} x} e^{-\frac{1}{2}(x-\mu)^{T} \Sigma^{-1}(x-\mu)} d x \\
& =\frac{1}{\operatorname{det}(\Sigma)^{1 / 2}(2 \pi)^{n / 2}} \int f(x) e^{-\frac{1}{2}(x-(\mu+i \Sigma u))^{T} \Sigma^{-1}(x-(\mu+i \Sigma u))} e^{i u^{T} \mu-\frac{1}{2} u^{T} \Sigma u} d x
\end{aligned}
$$


Now with a change of variables $y=x-i \Sigma u$, we obtain:

$$
\begin{aligned}
\mathbb{E}\left(f(x) e^{i u^{T} x}\right) & \left.=\frac{1}{\operatorname{det}(\Sigma)^{1 / 2}(2 \pi)^{n / 2}} e^{i u^{T} \mu-\frac{1}{2} u^{T} \Sigma u} \int f(y+i \Sigma u)\right) e^{-\frac{1}{2}(y-\mu)^{T} \Sigma^{-1}(y-\mu)} d y \\
& \left.=e^{i u^{T} \mu-\frac{1}{2} u^{T} \Sigma u} \mathbb{E}(f(y+i \Sigma u))\right)
\end{aligned}
$$

Note: technically we require that $\mathbb{E}(|f(x)|)<\infty$ with respect to a Gaussian measure so as to apply the dominated convergence theorem, and an analytic extension of the Gaussian integral to complex numbers, but these arguments are standard and we omit them (see for example [46]).

Lemma 7.2. Let $x \in \mathbb{R}^{n}$ be drawn from a mixture of $k$ spherical Gaussians in $\mathbb{R}^{n}, u, z \in \mathbb{R}^{n}$ as in the algorithm. Let $\hat{w}_{j}=w_{j} e^{i u^{T} \mu_{j}-\frac{1}{2} \sigma_{j}^{2}\|u\|^{2}}$. Then,

$$
\begin{aligned}
\mathbb{E}\left(x x^{T}\right) & =\sum_{j=1}^{k} w_{j} \sigma_{i}^{2} I+\sum_{j=1}^{k} w_{j} \mu_{j} \mu_{j}^{T} . \\
\mathbb{E}\left(x x^{T} e^{i u^{T} x}\right) & =\sum_{j=1}^{k} \hat{w}_{j} \sigma_{j}^{2} I+\sum_{j=1}^{k} \hat{w}_{j}\left(\mu_{j}+i \sigma_{j}^{2} u\right)\left(\mu_{j}+i \sigma_{j}^{2} u\right)^{T} . \\
\mathbb{E}\left(x\left(z^{T} x\right)^{2} e^{i u^{T} x}\right) & =\sum_{j=1}^{k} \hat{w}_{j} \sigma_{j}^{2}\left(\mu_{i}+i \sigma_{j}^{2} u\right) .
\end{aligned}
$$

Proof. These are obtained by direct calculation and expanding out $x_{i} \sim N\left(\mu_{i}, \sigma^{2} I_{n}\right)$. For (40):

$$
\begin{aligned}
\mathbb{E}\left(x x^{T}\right) & =\sum_{j=1}^{k} w_{j} \mathbb{E}_{F_{j}}\left(x x^{T}\right) \\
& =\sum_{j=1}^{k} w_{j} \mathbb{E}\left(\left(x-\mu_{j}+\mu_{j}\right)\left(x-\mu_{j}+\mu_{j}\right)^{T}\right) \\
& =\sum_{j=1}^{k} w_{j}\left[\sigma_{j}^{2} I_{n}+\mu_{j} \mu_{j}^{T}\right]
\end{aligned}
$$

(41) follows by applying Lemma 7.1 and the previous result:

$$
\begin{aligned}
\mathbb{E}\left(x x^{T} e^{i u^{T} x}\right) & =\sum_{i=1}^{k} w_{i} e^{i u^{T} \mu_{i}-\frac{1}{2} \sigma_{i}^{2}\|u\|^{2}} \mathbb{E}_{F_{i}}\left(\left(x_{i}+i \sigma_{i}^{2} u\right)\left(x_{i}+i \sigma_{i}^{2} u\right)^{T}\right) \\
& =\sum_{i=1}^{k} \hat{w}_{i}\left[\sigma_{i}^{2} I_{n}+\left(\mu_{i}+i \sigma_{i}^{2} u\right)\left(\mu_{i}+i \sigma_{i}^{2} u\right)^{T}\right]
\end{aligned}
$$


To see (42), we write (noting that $z$ is orthogonal to $u$ and to each $\mu_{j}$ ),

$$
\begin{aligned}
\mathbb{E}\left(x\left(z^{T} x\right)^{2} e^{i u^{T} x}\right) & =\sum_{j=1}^{k} \hat{w}_{j} \mathbb{E}\left(\left(x+i \sigma_{j}^{2} u\right)\left(z^{T}\left(x+i \sigma_{j}^{2} u\right)\right)^{2}\right) \\
& =\sum_{j=1}^{k} \hat{w}_{j} \mathbb{E}\left(\left(x-\mu_{j}+\mu_{j}+i \sigma_{j}^{2} u\right)\left(z^{T}\left(x-\mu_{j}\right)\right)^{2}\right) \\
& =\sum_{j=1}^{k} \hat{w}_{j}\left(\mathbb{E}\left(\left(x-\mu_{j}\right)\left(z^{T}\left(x-\mu_{j}\right)\right)^{2}\right)+\mathbb{E}\left(\left(\mu_{j}+i \sigma_{j}^{2} u\right)\left(z^{T}\left(x-\mu_{j}\right)\right)^{2}\right)\right) \\
& =\sum_{j=1}^{k} \hat{w}_{j} \sigma_{j}^{2}\left(\mu_{j}+i \sigma_{j}^{2} u\right) .
\end{aligned}
$$

Instead of polynomial anti-concentration under a Gaussian measure, we require only a simpler lemma concerning the anti-concentration of complex exponentials:

Lemma 7.3 (Complex exponential anti-concentration). Let $\mu_{i}, \mu_{j} \in \mathbb{R}^{n}$ satisfy $\left\|\mu_{i}-\mu_{j}\right\|>0$, then for $u \sim N\left(0, \sigma^{2} I_{n}\right)$ where $\left\|\mu_{i}-\mu_{j}\right\|^{2} \sigma^{2} \leq 2 \pi^{2}$. Then:

$$
\operatorname{Pr}\left(\left|e^{i \mu_{i}^{T} u}-e^{i \mu_{j}^{T} u}\right| \leq \epsilon\right) \leq \frac{16 \epsilon}{\left\|\mu_{i}-\mu_{j}\right\| \sigma \sqrt{2 \pi}}
$$

Proof. First, note that it suffices to show anti-concentration of the complex exponential around 1:

$$
\left|e^{i \mu_{i}^{T} u}-e^{i \mu_{j}^{T} u}\right|=\left|e^{i \mu_{i}^{T} u}\right|\left|1-e^{i\left(\mu_{i}-\mu_{j}\right)^{T} u}\right|=\left|1-e^{i\left(\mu_{i}-\mu_{j}\right)^{T} u}\right|
$$

The exponent $\left(\mu_{i}-\mu_{j}\right)^{T} u$ is of course a random variable $z \in \mathbb{R}$ distributed according to $N\left(0, \sigma^{2}\left\|\mu_{i}-\mu_{j}\right\|^{2}\right)$. From plane geometry, we know that: $\left|e^{i z}-1\right|>\epsilon$ in case

$$
z \notin \cup_{k \in \mathbb{Z}}[2 \pi k-2 \epsilon, 2 \pi k+2 \epsilon]
$$

We can bound this probability as follows:

$$
\begin{aligned}
\operatorname{Pr}\left(z \notin \cup_{k \in \mathbb{Z}}[2 \pi k-2 \epsilon, 2 \pi k+2 \epsilon]\right) & \leq 2 \sum_{k=0}^{\infty} \frac{4 \epsilon}{\left\|\mu_{i}-\mu_{j}\right\| \sigma \sqrt{2 \pi}} e^{-\frac{(2 \pi k)^{2}}{2\left\|\mu_{i}-\mu_{j}\right\|^{2} \sigma^{2}}} \\
& \leq \frac{8 \epsilon}{\left\|\mu_{i}-\mu_{j}\right\| \sigma \sqrt{2 \pi}} \sum_{k=0}^{\infty} e^{-\frac{2 \pi^{2} k}{\left\|\mu_{i}-\mu_{j}\right\|^{2} \sigma^{2}}} \\
& =\frac{8 \epsilon}{\left\|\mu_{i}-\mu_{j}\right\| \sigma \sqrt{2 \pi}} \frac{1}{1-e^{-\frac{2 \pi^{2}}{\left\|\mu_{i}-\mu_{j}\right\|^{2} \sigma^{2}}}} \\
& \leq \frac{16 \epsilon}{\left\|\mu_{i}-\mu_{j}\right\| \sigma \sqrt{2 \pi}}
\end{aligned}
$$

where the last line follows from the assumption $\left\|\mu_{i}-\mu_{j}\right\|^{2} \sigma^{2} \leq 2 \pi^{2}$.

We can now prove that the algorithm is correct with sufficiently many samples. Using PCA we can find the span of the means $\left\{\mu_{1}, \ldots, \mu_{k}\right\}$, as the span of the top $k-1$ right singular vectors of the matrix whose rows are sample points [64. Projecting to this space, the mixture remains a mixture of spherical Gaussians. We assume that the $\mu_{i}$ are linearly independent (as in recent work [39] with higher moments). 
Proof of Theorem 1.5. From Lemma 7.2, we observe that for any unit vector $v$,

$$
\mathbb{E}\left(\left(v^{T} x\right)^{2}\right)=v^{T} \mathbb{E}\left(x x^{T}\right) v=\sum_{i=1}^{k} w_{i} \sigma_{i}^{2}+\sum_{i=1}^{k} w_{i}\left(\mu_{i}^{T} v\right)^{2} .
$$

Without loss of generality, we may assume that the overall mean is 0 , hence $0=\sum_{i} w_{i} \mu_{i}$ is 0 and therefore the $\mu_{i}$ are linearly dependent, and there exist a $v$ orthogonal to all the $\mu_{i}$. For such a $v$, the variance is $\sigma^{2}=\sum_{i=1}^{k} w_{i} \sigma_{i}^{2}$ while for $v$ in the span, the variance is strictly higher. Therefore the value $\sigma^{2}$ is simply the $k^{\prime}$ th eigenvalue of $\mathbb{E}\left(x x^{T}\right)$ (assuming $x$ is centered at 0 ).

Thus, in the algorithm we have estimated the matrices

$$
M=\sum_{i=1}^{k} w_{i} \mu_{i} \mu_{i}^{T}=A A^{T} \text { and } M_{u}=\sum_{i=1}^{k} w_{i} e^{-\frac{1}{2}\|u\|^{2} \sigma_{i}^{2}+i u^{T} \mu_{i}} \mu_{i} \mu_{i}^{T}=A D_{u} A^{T} .
$$

with $\left(D_{u}\right)_{i i}=e^{-\frac{1}{2}\|u\|^{2} \sigma_{i}^{2}+i u^{T} \mu_{i}}$. Thus,

$$
M_{u} M^{-1}=A D_{u} A^{-1}
$$

and its eigenvectors are the columns of $A$, assuming the entries of $D_{u}$ are distinct. We note that the columns of $A$ are precisely $\tilde{\mu}_{j}=\sqrt{w_{j}} \mu_{j}$. The eigenvalue corresponding to the eigenvector $\tilde{\mu}_{j}$ is the $j$ 'th diagonal entry of $D_{u}$.

To prove the algorithm's correctness, we will once again apply Theorem 5.4 for robust tensor decomposition by verifying its five conditions. Condition 1 holds by our assumption on the means of the Gaussian mixtures. Conditions 3 holds by taking sufficiently many samples (the overall sample and time complexity will be linear in $n$ and polynomial in $k$ ), Conditions 2 and 4 hold by applying 7.3 .

We can apply our observations regarding Gaussian noise from Section 4.8. Namely, the covariance of the reweighted Gaussian is shifted by $\Sigma_{\eta}$, the covariance of the unknown noise. Thus, by considering $\Sigma_{u}$ and the standard covariance, and taking their difference, the contribution of the noise is removed and we are left with a matrix that can be diagonalized using $A$.

\section{Perturbation bounds}

In this section, we collect all the eigenvalue decomposition bounds that we require in our proofs. The generalized Weyl inequality we derive in Theorem 8.5 surprisingly seems to be unknown in the literature.

\subsection{SVD perturbations}

In this section, we present two standard perturbation bounds on singular vectors. These bounds will help determine the accuracy needed in estimating the matrix with samples.

Lemma 8.1. Let $A \in \mathbb{C}^{n \times n}$ and suppose that $\sigma_{i}(A)-\sigma_{i+1}(A) \geq \epsilon$ for all $i$. Let $E \in \mathbb{C}^{n \times n}$ be a matrix where where $\|E\|_{2} \leq \delta$. Denote by $v_{i}$ the right singular vectors of $A$ and $\hat{v}_{i}$ the right singular vectors of $A+E$, then:

$$
\left\|v_{i}-\hat{v}_{i}\right\| \leq \frac{\sqrt{2} \delta}{\epsilon-\delta}
$$

Proof. We first write:

$$
\left\|v_{i}-\hat{v}_{i}\right\|^{2}=\left\langle v_{i}-\hat{v}_{i}, v_{i}-\hat{v}_{i}\right\rangle=2\left(1-\left\langle v_{i}, \hat{v}_{i}\right\rangle\right)=2(1-\cos (\theta)) \leq 2\left(1-\cos (\theta)^{2}\right)=2 \sin (\theta)^{2}
$$

Next, we apply the following form of Wedin's Theorem from [62] where notions such as the canonical angles etc. used in the statement below are also explained. 
Theorem 8.2. Let $A, E \in \mathbb{C}^{m \times n}$ be complex matrices with $m \geq n$. Let $A$ have singular value decomposition

$$
A=\left[U_{1} U_{2} U_{3}\right]\left(\begin{array}{cc}
\Sigma_{1} & 0 \\
0 & \Sigma_{2} \\
0 & 0
\end{array}\right)\left[V_{1}^{*} V_{2}^{*}\right]
$$

and similarly for $\tilde{A}=A+E$ (with conformal decomposition using $\tilde{U}_{1}, \tilde{\Sigma}_{1}$ etc). Suppose there are numbers $\alpha, \beta>0$ such that

1. $\min \sigma\left(\tilde{\Sigma}_{1}\right) \geq \alpha+\beta$,

2. $\max \sigma\left(\Sigma_{2}\right) \leq \alpha$.

Then

$$
\|\sin (\Phi)\|_{2},\|\sin (\Theta)\|_{2} \leq \frac{\|E\|_{2}}{\beta}
$$

where $\Phi$ is the (diagonal) matrix of canonical angles between the ranges of $U_{1}$ and $\tilde{U}_{1}$ and $\Theta$ denotes the matrix of canonical angles between the ranges of $U_{2}$ and $\tilde{U}_{2}$.

We also require the following form of Weyl's Inequality (see [62]):

Lemma 8.3. Let $A, E \in \mathbb{C}^{m \times n}$, then

$$
\left|\sigma_{i}(A+E)-\sigma_{i}(A)\right| \leq \sigma_{1}(E)
$$

By Weyl's inequality, we know that $\left|\sigma\left(\Sigma_{1}\right)-\sigma\left(\tilde{\Sigma}_{2}\right)\right| \geq \epsilon-\delta$. Similarly for the smallest singular value. By Wedin's theorem, we pick the partition $\Sigma_{1}$ to be the top $i$ singular values, with $\Sigma_{2}$ the remaining ones. Thus, taking $\alpha=\sigma_{i+1}(A)$ and $\beta=\epsilon-\delta$, we have

$$
|\sin (\theta)| \leq\|\sin (\Phi)\|_{2} \leq \frac{\delta}{\epsilon-\delta}
$$

as required.

\subsection{Perturbations of complex diagonalizable matrices}

The main technical issue in giving a robust version of our algorithms is that the stability of eigenvectors of general matrices is more complicated than for Hermitian or normal matrices where the $\sin (\theta)$ theorem of Davis and Kahan 31 describes the whole situation. Roughly speaking, the difficulty lies in the fact that for a general matrix, the eigenvalue decomposition is given by $A=P D P^{-1}$. Upon adding a perturbation $E$, it is not clear a priori that $A+E$ has a full set of eigenvectors - that is to say, $A+E$ may no longer be diagonalizable. The goal of this section is to establish that for a general matrix with well-spaced eigenvalues, sufficiently small perturbations do not affect the diagonalizability. We use Bauer-Fike theorem via a homotopy argument typically used in proving strong versions of the Gershgorin Circle Theorem 68.

Theorem 8.4 (Bauer-Fike [11]). Let $A \in \mathbb{C}^{n \times n}$ be a diagonalizable matrix such that $A=X \operatorname{diag}\left(\lambda_{i}\right) X^{-1}$. Then for any eigenvalue $\mu$ of $A+E \in \mathbb{C}^{n \times n}$ we have

$$
\min _{i}\left|\lambda_{i}(A)-\mu\right| \leq \kappa(X)\|E\|_{2}
$$

Using this, we prove a weak version of Weyl's theorem for diagonalizable matrices whose eigenvalues are well-spaced. We consider this a spectral norm version of the strong Gershgorin Circle theorem (which uses row-wise $L^{1}$ norms). 
Lemma 8.5 (Generalized Weyl inequality). Let $A \in \mathbb{C}^{n \times n}$ be a diagonalizable matrix such that $A=$ $X \operatorname{diag}\left(\lambda_{i}\right) X^{-1}$. Let $E \in \mathbb{C}^{n \times n}$ be a matrix such that $\left|\lambda_{i}(A)-\lambda_{j}(A)\right| \geq 3 \kappa(X)\|E\|_{2}$ for all $i \neq j$. Then there exists a permutation $\pi:[n] \rightarrow[n]$ such that

$$
\left|\lambda_{i}(A+E)-\lambda_{\pi(i)}(A)\right| \leq \kappa(X)\|E\|_{2} .
$$

Proof. Consider the matrix $M(t)=A+t E$ for $t \in[0,1]$. By the Bauer-Fike theorem, every eigenvalue $\hat{\lambda}(t)$ of $M(t)$ is contained in $\mathbb{B}\left(\lambda_{i}, t \kappa(X)\|E\|_{2}\right.$ ) for some $i$ (for $\lambda \in \mathbb{C}, t \in \mathbb{R}$ we use $\mathbb{B}(\lambda, t)$ to denote the ball in $\mathbb{C}$ of radius $t$ with center at $\lambda)$. In particular, when $t=0$ we know that $\hat{\lambda}(0)=\lambda_{i} \in \mathbb{B}\left(\lambda_{i}, 0\right)$.

As we increase $t, \hat{\lambda}(t)$ is a continuous function of $t$, thus it traces a connected curve in $\mathbb{C}$. Suppose that $\hat{\lambda}(1) \in \mathbb{B}\left(\lambda_{j}, \kappa(X)\|E\|_{2}\right)$ for some $j \neq i$, then for some $t^{*}$, we must have $\hat{\lambda}\left(t^{*}\right) \notin \bigcup_{j} \mathbb{B}\left(\lambda_{i}, \kappa(X)\|E\|_{2}\right)$ as these balls are disjoint. This contradicts the Bauer-Fike theorem. Hence we must have $\hat{\lambda}(1) \in \mathbb{B}\left(\lambda_{i}, \kappa(X)\|E\|_{2}\right)$ as desired.

The following is a sufficient condition for the diagonalizability of a matrix. The result is well-known (Exercise V.8.1 in [4] for example).

Lemma 8.6. Let $A: V \rightarrow V$ be a linear operator over a finite dimensional vector space of dimension $n$. Suppose that all the eigenvalues of $A$ are distinct, i.e., $\lambda_{i} \neq \lambda_{j}$ for all pairs $i, j$. Then $A$ has $n$ linearly independent eigenvectors.

We require the following generalisation of the Davis-Kahan $\sin (\theta)$ theorem 31 for general diagonalizable matrices due to Eisenstat and Ipsen 34:

Theorem 8.7 (Generalized $\sin (\theta)$ Theorem). Let $A, A+E \in \mathbb{C}^{n \times n}$ be diagonalizable matrices. Let $\hat{\gamma}$ be an eigenvalue of $A+E$ with associated eigenvector $\hat{x}$. Let

$$
A=\left[X_{1}, X_{2}\right]\left(\begin{array}{cc}
\Gamma_{1} & 0 \\
0 & \Gamma_{2}
\end{array}\right)\left[X_{1}, X_{2}\right]^{-1},
$$

be an eigendecomposition of $A$. Here $\Gamma_{1}$ consists of eigenvalues of $A$ closest to $\hat{\gamma}$, i.e. $\left\|\Gamma_{1}-\hat{\gamma} I\right\|_{2}=$ $\min _{i}\left|\gamma_{i}-\hat{\gamma}\right|$, with associated matrix of eigenvectors $X_{1}$. And $\Gamma_{2}$ contains the remaining eigenvalues and the associated eigenvectors are in $X_{2}$. Also let $\left[X_{1}, X_{2}\right]^{-1}=:\left(\begin{array}{c}Z_{1}^{*} \\ Z_{2}^{*}\end{array}\right)$.

Then the angle between $\hat{x}$ and the subspace spanned by the eigenvectors associated with $\Gamma_{1}$ is given by

$$
\sin (\theta) \leq \kappa\left(Z_{2}\right) \frac{\|(A-\hat{\gamma} I) \hat{x}\|_{2}}{\min _{i}\left|\left(\Gamma_{2}\right)_{i i}-\hat{\gamma}\right|} .
$$

\section{General position and average case analysis}

Our necessary condition for identifiability is satisfied almost surely by randomly chosen vectors for a fairly general class of distributions. For simplicity we restrict ourselves to the case of $d=2$ and Gaussian distribution in the following theorem; the proof of a more general statement would be similar.

Theorem 9.1. Let $v_{1}, \ldots, v_{m} \in \mathbb{R}^{n}$ be standard Gaussian i.i.d. random vectors, with $m \leq\left(\begin{array}{c}n+1 \\ 2\end{array}\right)$. Then $v_{1}^{\otimes 2}, \ldots, v_{m}^{\otimes 2}$ are linearly independent almost surely.

Proof Sketch. Let's take $m=\left(\begin{array}{c}n+1 \\ 2\end{array}\right)$ without loss of generality. Consider vextors $w_{1}, \ldots, w_{m}$, where $w_{i}$ is obtained from $v_{i}^{\otimes 2}$ by removing duplicate components; e.g., for $v_{1} \in \mathbb{R}^{2}$, we have $v_{1}^{\oplus 2}=\left(v_{1}(1)^{2}, v_{1}(2)^{2}, v_{1}(1) v_{1}(2), v_{2}(1) v_{1}(2)\right)$

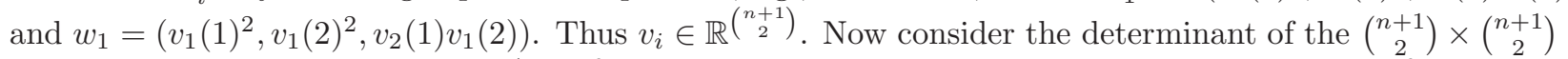
matrix with the $w_{i}$ as columns. As a formal multivariate polynomial with the components of the $v_{i}$ as variables, this determinant is not identically 0 . This is because, for example, it can be checked that the monomial $w_{1}(1)^{2} \ldots w_{n}(n)^{2} w_{n+1}(\rho(n+1)) \ldots w_{m}(\rho(m))$ occurs precisely once in the expansion of the determinant as a sum of monomials (here $\rho:\{n+1, \ldots, m\} \rightarrow\left(\begin{array}{c}{[n]} \\ 2\end{array}\right)$ is an arbitrary bijection). The proof can now be completed along the lines of the well-known Schwartz-Zippel lemma. 
We now show that the condition number of the Khatri-Rao power of a random matrix behaves well in certain situations. For simplicity we will deal with the case where the entries of the base matrix $M$ are chosen from $\{-1,1\}$ uniformly at random; the case of Gaussian entries also gives a similar though slightly weaker result, but would require some extra work.

We define a notion of $d^{\prime}$ 'th power of a matrix $M \in \mathbb{R}^{n \times m}$ which is similar to the Khatri-Rao power except that we only keep the non-redundant multilinear part resulting in $\left(\begin{array}{l}n \\ d\end{array}\right) \times m$ matrix. Working with this multilinear part will simplify things. Formally, $M^{\ominus d}:=\left[M_{1}^{\ominus d}, \ldots, M_{m}^{\ominus d}\right]$, where for a column vector $C \in \mathbb{R}^{n}$, define $C^{\ominus d} \in \mathbb{R}^{\left(\begin{array}{l}n \\ d\end{array}\right)}$ with entries given by $C_{S}:=C_{i_{1}} C_{i_{2}} \ldots C_{i_{d}}$ where where $1 \leq i_{1}<i_{2}<\ldots<i_{d} \leq n$ and $S=\left\{i_{1}, \ldots, i_{d}\right\} \in\left(\begin{array}{c}{[n]} \\ d\end{array}\right)$.

The following theorem is stated for the case when the base matrix $M \in \mathbb{R}^{n \times n^{2}}$. This choice is to keep the statement and proof of the theorem simple; generalization to more general parameterization is straightforward. While the theorem below is proved for submatrices $M^{\ominus d}$ of the Khatri-Rao power $M^{\odot d}$, similar results hold for $M^{\odot d}$ by the interlacing properties of the singular values of submatrices [63.

Theorem 9.2. Let $M \in \mathbb{R}^{n \times m}$ be chosen by sampling each entry iid uniformly at random from $\{-1,1\}$. For $m=n^{2}$, integer $d \geq 3$, and $N=\left(\begin{array}{l}n \\ d\end{array}\right)$, and $A=M^{\ominus d}$ we have

$$
\mathbb{E} \max _{j \leq n^{2}}\left|\sigma_{j}(A)-\sqrt{N}\right|<N^{1 / 2-\Omega(1)} .
$$

Proof. We are going to use Theorem 5.62 of Vershynin [66] which we state here essentially verbatim:

Theorem 9.3 ([66]). Let $A$ be an $N \times m$ matrix $(N \geq m)$ whose columns $A_{j}$ are independent isotropic random vectors in $\mathbb{R}^{N}$ with $\left\|A_{j}\right\|_{2}=\sqrt{N}$ almost surely. Consider the incoherence parameter

$$
\mu:=\frac{1}{N} \mathbb{E} \max _{j \leq m} \sum_{k \in[m], k \neq j}\left\langle A_{j}, A_{k}\right\rangle^{2} .
$$

Then for absolute constants $C, C_{0}$ we have $\mathbb{E}\left\|\frac{1}{N} A^{*} A-I\right\| \leq C_{0} \sqrt{\frac{\mu \log m}{N}}$. In particular,

$$
\mathbb{E} \max _{j \leq m}\left|\sigma_{j}(A)-\sqrt{N}\right|<C \sqrt{\mu \log m}
$$

Our matrix $A=M^{\ominus d}$ will play the role of matrix $A$ in Theorem 9.3. Note that for a column $A_{j}$ we have $\mathbb{E} A_{j} \otimes A_{j}=I$, so the $A_{j}$ are isotropic. Also note that $\left\|A_{j}\right\|_{2}=\sqrt{N}$ always.

We now bound the incoherence parameter $\mu$. To this end, we first prove a concentration bound for $\left\langle A_{j}, A_{k}\right\rangle$, for fixed $j, k$. We use a concentration inequality for polynomials of random variables. Specifically, we use Theorem 23 at (http://www.contrib.andrew.cmu.edu/ ryanod/?p=1472). Let us restate that theorem here.

Theorem 9.4. Let $f:\{-1,1\}^{n} \rightarrow \mathbb{R}$ be a polynomial of degree at most $k$. Then for any $t \geq(2 e)^{k / 2}$ we have

$$
\operatorname{Pr}_{x \sim\{-1,1\}}\left[|f(x)| \geq t\|f\|_{2}\right] \leq \exp \left(-\frac{k}{2 e} t^{2 / k}\right) .
$$

Here $\|f\|_{2}:=\left[\mathbb{E}_{x} f(x)^{2}\right]^{1 / 2}$. For our application to $\left\langle A_{j}, A_{k}\right\rangle$, we first fix $A_{j}$ arbitrarily. Then $\left\langle A_{j}, A_{k}\right\rangle$, which will play the role of $f(x)$ in the above theorem, can be written as $\sum_{S \in\left(\begin{array}{c}{[n]} \\ d\end{array}\right)} c_{S} x_{S}$ where the choice of the coefficients $c_{S}= \pm 1$ comes from the fixing of $A_{j}$ and the entries of $A_{k}$ are of the form $x_{S}$, where $S \in\left(\begin{array}{c}{[n]} \\ d\end{array}\right)$. Now 


$$
\begin{aligned}
\mathbb{E}_{x}\left\langle A_{j}, A_{k}\right\rangle^{2} & =\sum_{S, S^{\prime} \in\left(\begin{array}{c}
{[n]} \\
d
\end{array}\right)} c_{S} c_{s^{\prime}} \mathbb{E}_{x} x_{S} x_{S^{\prime}} \\
& =\sum_{S \in\left(\begin{array}{c}
{[n]} \\
d
\end{array}\right)} c_{S}^{2} \mathbb{E}_{x} x_{S}^{2}+\sum_{S, S^{\prime} \in\left(\begin{array}{c}
{[n]} \\
d
\end{array}\right): S \neq S^{\prime}} c_{S} c_{S^{\prime}} \mathbb{E}_{x} x_{S} x_{S^{\prime}} \\
& =\left(\begin{array}{c}
n \\
d
\end{array}\right) \\
& =N .
\end{aligned}
$$

In other words, for our choice of $f$ we have $\|f\|_{2}=\sqrt{N}$.

Applying Theorem 9.4 with $t \geq(2 e)^{d / 2}$ and $\lambda=t \sqrt{N}$ we have

$$
\operatorname{Pr}_{x \sim\{-1,1\}}\left[\left|\left\langle A_{j}, A_{k}\right\rangle\right| \geq \lambda\right] \leq \exp \left(-\frac{d}{2 e} t^{2 / d}\right)=\exp \left(-\frac{d}{2 e} \frac{\lambda^{2 / d}}{N^{1 / d}}\right) .
$$

Note that we proved the above inequality for any fixed $A_{j}$, so clearly it also follows when $A_{j}$ is also random.

We now estimate parameter $\mu$. Note that $\left\langle A_{j}, A_{k}\right\rangle^{2} \leq N^{2}$ always. When the union of the event in (43) over all $j \neq k$, which we denote by $B$, does not hold, we will use the bound just mentioned. For the following computation recall that the number of columns $m$ in $A$ is $n^{2}$.

$$
\begin{aligned}
\mu & \leq \frac{1}{N} m \lambda^{2} \operatorname{Pr}(\bar{B})+\frac{1}{N} m N^{2} \operatorname{Pr}(B) \\
& \leq \frac{m \lambda^{2}}{N}+\frac{m\left(\begin{array}{c}
m \\
2
\end{array}\right) N^{2}}{N} \exp \left(-\frac{d}{2 e} \frac{\lambda^{2 / d}}{N^{1 / d}}\right) \\
& \leq \frac{n^{2} \lambda^{2}}{N}+n^{6} N \exp \left(-\frac{d}{2 e} \frac{\lambda^{2 / d}}{N^{1 / d}}\right) .
\end{aligned}
$$

Now choose $\lambda:=N^{1 / 2+\epsilon}$ for a small $\epsilon>0$. Then the expression in (44) is bounded by

$$
\text { (44) } \leq n^{2} N^{2 \epsilon}+n^{6} N \exp \left(-\frac{d}{2 e} N^{2 \epsilon / d}\right) \text {. }
$$

It's now clear that for a sufficiently small choice of $\epsilon$ (say 0.05) and sufficiently large $n$ (cepending on $d$ and $\epsilon$ ), only the first term above is significant and using our assumption $d>2$ gives

$$
\mu<2 n^{2} N^{2 \epsilon}<2 d ! N^{2 / d+\epsilon}<<N .
$$

Therefore by Theorem 9.3 we have

$$
\mathbb{E}\left\|\frac{1}{N} A^{*} A-I\right\| \leq C_{0} \sqrt{\frac{\mu \log n^{2}}{N}}<1 / N^{\Omega(1)},
$$

which gives

$$
\mathbb{E} \max _{j \leq n^{2}}\left|\sigma_{j}(A)-\sqrt{N}\right|<N^{1 / 2-\Omega(1)} .
$$

In particular, setting $s_{\min }(A):=s_{n^{2}}(A)$ we have

$$
\mathbb{E}\left|\sigma_{\min }(A)-\sqrt{N}\right|<1 / N^{1 / 2-\Omega(1)} .
$$

Using Markov this also gives probability bounds. 


\section{Technical lemmas}

In this section we collect some of the technical claims needed in the paper.

Lemma 10.1 (Nonvanishing of $\phi(t)$ ). Let $s$ be a real-valued random vector in $\mathbb{R}^{m}$ with independent components and $\mathbb{E}(s)=0$. Also let $\mathbb{E}\left(\left|s_{j}\right|\right)$ and $\mathbb{E}\left(\left|s_{j}^{2}\right|\right)$ exist and $\mathbb{E}\left(\left|s_{j}^{2}\right|\right) \leq M_{2}$ for all $j$ for $M_{2}>0$. Then for $t \in \mathbb{R}^{m}$ with $\|t\|_{2} \leq \frac{1}{2 \sqrt{M_{2}}}$ the characteristic function $\phi(\cdot)$ of s satisfies $|\phi(t)| \geq 3 / 4$.

Proof. Using Taylor's theorem 4.8 for $\cos y$ and $\sin y$ gives

$$
e^{i y}=\cos y+i \sin y=1+i y-\frac{(i y)^{2}}{2 !}\left[\cos \left(\theta_{1} y\right)+i \sin \left(\theta_{2} y\right)\right]
$$

for $y, \theta_{1}, \theta_{2} \in \mathbb{R}$ with $\left|\theta_{1}\right| \leq 1,\left|\theta_{2}\right| \leq 1$. Applying this to $y=t^{T} s$, taking expectation over $s$, and using the assumption of zero means on the $s_{i}$ we get

$$
\mathbb{E}\left(e^{i t^{T} s}\right)=1-\mathbb{E}\left(\frac{\left(i t^{T} s\right)^{2}}{2}\left[\cos \left(\theta_{1} y\right)+i \sin \left(\theta_{2} y\right)\right]\right),
$$

which using the indpendence of the components of $s$ and the zero means assumption gives

$$
\begin{aligned}
\left|\mathbb{E}\left(e^{i t^{T} s}\right)-1\right|=|\phi(t)-1| & \leq \frac{1}{2} \mathbb{E}\left(\left(t^{T} s\right)^{2}\left|\cos \left(\theta_{1} y\right)+i \sin \left(\theta_{2} y\right)\right|\right) \\
& \leq \mathbb{E}\left(\left(t^{T} s\right)^{2}\right) \\
& =\sum_{j} t_{j}^{2} \mathbb{E}\left(s_{j}^{2}\right) \\
& \leq R_{2}\|t\|_{2}^{2} \\
& \leq 1 / 4
\end{aligned}
$$

Lemma 10.2. Let $a_{1}, \ldots, a_{d}, b_{1}, \ldots, b_{d} \in \mathbb{C}$ be such that $\left|a_{j}-b_{j}\right| \leq \epsilon$ for real $\epsilon \geq 0$, and $\left|a_{j}\right| \leq R$ for $R>0$. Then

$$
\left|\prod_{j=1}^{d} a_{j}-\prod_{j=1}^{d} b_{j}\right| \leq(R+\epsilon)^{d}-R^{d} .
$$

Proof. For $0<j<d$, define the $j$ th elementary symmetric function in $d$ variables: $\sigma_{j}\left(x_{1}, \ldots, x_{d}\right)=$ $\sum_{1 \leq i_{1} \leq \ldots \leq i_{j} \leq d} x_{i_{1}} \ldots x_{i_{j}}$. We will use the following well-known inequality (see, e.g., 61]) which holds for $x_{\ell} \geq 0$ for all $\ell$.

$$
\left(\frac{\sigma_{j}\left(x_{1}, \ldots, x_{d}\right)}{\left(\begin{array}{l}
d \\
j
\end{array}\right)}\right)^{1 / j} \leq \frac{\sigma_{1}\left(x_{1}, \ldots, x_{d}\right)}{d}
$$

Let $b_{j}=a_{j}+\epsilon_{j}$. Then

$$
\begin{aligned}
\left|\prod_{j}\left(a_{j}+\epsilon_{j}\right)-\prod_{j} a_{j}\right| & \leq \epsilon \sigma_{d-1}\left(\left|a_{1}\right|, \ldots,\left|a_{d}\right|\right)+\epsilon^{2} \sigma_{d-2}\left(\left|a_{1}\right|, \ldots,\left|a_{d}\right|\right)+\ldots+\epsilon^{d-1} \sigma_{1}\left(\left|a_{1}\right|, \ldots,\left|a_{d}\right|\right) \\
& \leq d \epsilon R^{d-1}+\left(\begin{array}{c}
d \\
2
\end{array}\right) \epsilon^{2} R^{d-2}+\ldots+\epsilon^{d} \\
& =(R+\epsilon)^{d}-R^{d}
\end{aligned}
$$

where the second inequality follows from (45). 
Claim 10.3. Let $u \in \mathbb{R}$ be sampled according to $N\left(0, \sigma^{2}\right)$. Then for $\tau>0$ we have

$$
\operatorname{Pr}(|u|>\tau) \leq \sqrt{\frac{2}{\pi}} \frac{\sigma^{2}}{\tau} e^{-\frac{\tau^{2}}{2 \sigma^{2}}}
$$

Proof. Follows from the well-known fact: $\frac{1}{\sqrt{2 \pi}} \int_{a}^{\infty} e^{-z^{2} / 2} d z \leq \frac{1}{\sqrt{2 \pi}} \cdot \frac{1}{a} \cdot e^{-a^{2} / 2}$, for $a>0$

We state the following easy claim without proof.

Claim 10.4. Let $B \in \mathbb{C}^{p \times m}$ with $p \geq m$ and colspan $(B)=m$. Let $D \in \mathbb{C}^{m \times m}$ be a diagonal matrix. Then

$$
\sigma_{m}\left(B D B^{T}\right) \geq \sigma_{m}(B)^{2} \sigma_{m}(D) .
$$

Claim 10.5. For $E \in \mathbb{C}^{m \times m}$ with $\|E\|_{F}<1 / 2$ we have

$$
(I-E)^{-1}=I+E+R,
$$

where $\|R\|_{F}<m\|E\|_{F}$.

Proof. For $\|E\|_{F}<1 / 2$ we have

$$
(I-E)^{-1}=I+E+E^{2}+\ldots
$$

Hence

$$
\left\|(I-E)^{-1}-(I+E)\right\|_{F} \leq\left\|E^{2}\right\|_{F}\left\|(I-E)^{-1}\right\|_{F}<m\|E\|_{F} .
$$

Fact 10.6. For a real-valued random variable $x$ and for any $0<p \leq q$ we have

$$
\begin{aligned}
\mathbb{E}\left(|x|^{p}\right)^{1 / p} & \leq \mathbb{E}\left(|x|^{q}\right)^{1 / q}, \\
\mathbb{E}\left(|x|^{p}\right) \mathbb{E}\left(|x|^{q}\right) & \leq \mathbb{E}\left(|x|^{p+q}\right) .
\end{aligned}
$$

Proof. Hölder's inequality implies that for $0 \leq p \leq q$ we have

$$
\mathbb{E}\left(|x|^{p}\right)^{1 / p} \leq \mathbb{E}\left(|x|^{q}\right)^{1 / q},
$$

and hence

$$
\mathbb{E}\left(|x|^{p}\right) \mathbb{E}\left(|x|^{q}\right) \leq \mathbb{E}\left(|x|^{p+q}\right)^{p /(p+q)} \mathbb{E}\left(|x|^{p+q}\right)^{q /(p+q)}=\mathbb{E}\left(|x|^{p+q}\right) .
$$

\section{Conclusion}

We conclude with some open problems. (1) Our condition for ICA to be possible required that there exist a $d$ such that $A^{\odot d}$ has full column rank. As mentioned before, the existence of such a $d$ turns out to be equivalent to the necessary and sufficient condition for ICA, namely, any two columns of $A$ are linearly independent. Thus if $d$ is large for a matrix $A$ then our algorithm whose running time is exponential in $d$ will be inefficient. This is inevitable to some extent as suggested by the ICA lower bound in [7]. However, the lower bound there requires that one of the $s_{i}$ be Gaussian. Can one prove the lower bound without this requirement? (2) Give an efficient algorithm for independent subspace analysis. This is the problem where the $s_{i}$ are not all indendent but rather the set of indices $[\mathrm{m}]$ is partitioned into subsets. For any two distinct subsets $S_{1}$ and $S_{2}$ in the partition $s_{S_{1}}$ is independent of $s_{S_{2}}$, where $s_{S_{1}}$ denotes the vector of the $s_{i}$ with $i \in S_{1}$ etc. Clearly this problem is a generalization of ICA.

Acknowledgements. We thank Sham Kakade for helpful discussions and Yongshun Xiao for showing us the Gershgorin Circle theorem and the continuous deformation technique used in Lemma 8.5 


\section{References}

[1] R. Adamczak, A. Litvak, A. Pajor, and N. Tomczak-Jaegermann. Quantitative estimates of the convergence of the empirical covariance matrix in logconcave ensembles. J. Amer. Math. Soc., 233:535-561, 2011.

[2] L. Albera, A. Ferréol, P. Comon, and P. Chevalier. Blind identification of overcomplete mixtures of sources (biome). Linear algebra and its applications, 391:3-30, 2004.

[3] A. Anandkumar, D. Foster, D. Hsu, S. Kakade, and Y.-K. Liu. A spectral algorithm for latent dirichlet allocation. In Advances in Neural Information Processing Systems 25, pages 926-934, 2012.

[4] A. Anandkumar, R. Ge, D. Hsu, S. Kakade, and M. Telgarsky. Tensor decompositions for learning latent variable models. 2012.

[5] A. Anandkumar, R. Ge, D. Hsu, S. M. Kakade, and M. Telgarsky. Tensor decompositions for learning latent variable models. CoRR, abs/1210.7559, 2012.

[6] A. Anandkumar, D. Hsu, and S. M. Kakade. A method of moments for mixture models and hidden markov models. In Proc. of COLT, 2012.

[7] J. Anderson, M. Belkin, N. Goyal, L. Rademacher, and J. Voss. The more, the merrier: the blessing of dimensionality for learning large gaussian mixtures. arXiv:1311.2891, 2013.

[8] J. Anderson, N. Goyal, and L. Rademacher. Efficient learning of simplices. COLT, 2013.

[9] S. Arora, R. Ge, A. Moitra, and S. Sachdeva. Provable ICA with unknown gaussian noise, with implications for gaussian mixtures and autoencoders. In NIPS, pages 2384-2392, 2012.

[10] R. I. Arriaga and S. Vempala. An algorithmic theory of learning: Robust concepts and random projection. Machine Learning, 63(2):161-182, 2006.

[11] F. L. Bauer and C. Fike. Norms and exclusion theorems. Numerische Mathematik, 2(1):137-141, 1960.

[12] M. Belkin, L. Rademacher, and J. Voss. Blind signal separation in the presence of Gaussian noise. In Proc. of COLT, 2013.

[13] M. Belkin and K. Sinha. Polynomial learning of distribution families. In FOCS, pages 103-112, 2010.

[14] M. Belkin and K. Sinha. Toward learning gaussian mixtures with arbitrary separation. In COLT, pages 407-419, 2010.

[15] A. Bhaskara, M. Charikar, A. Moitra, and A. Vijayaraghavan. Smoothed analysis of tensor decompositions. CoRR, abs/1311.3651, 2013.

[16] R. Bhatia. Matrix analysis, volume 169. Springer, 1997.

[17] P. Borwein and T. Erdelyi. Polynomials and Polynomial Inequalities. Springer, 1995.

[18] S. C. Brubaker and S. Vempala. Random tensors and planted cliques. In RANDOM, pages 406-419, 2009.

[19] S. C. Brubaker and S. S. Vempala. Isotropic PCA and affine-invariant clustering. In Building Bridges, pages 241-281. Springer, 2008.

[20] A. Carbery and J. Wright. Distributional and $L^{q}$ norm inequalities for polynomials over convex bodies in $R^{n}$. Mathematical Research Letters, 8:233-248, 2001.

[21] J. Cardoso. Source separation using higher order moments. In International Conference on Acoustics, Speech, and Signal Processing, 1989. 
[22] J. Cardoso. Super-symmetric decomposition of the fourth-order cumulant tensor. blind identification of more sources than sensors. In Acoustics, Speech, and Signal Processing, 1991. ICASSP-91., 1991 International Conference on, pages 3109-3112. IEEE, 1991.

[23] J. D. Carroll and J.-J. Chang. Analysis of individual differences in multidimensional scaling via an n-way generalization of Eckart-Young decomposition. Psychometrika, 35(3):283-319, 1970.

[24] J. T. Chang. Full reconstruction of markov models on evolutionary trees: identifiability and consistency. Mathematical biosciences, 137(1):51-73, 1996.

[25] K. Chaudhuri and S. Rao. Learning mixtures of product distributions using correlations and independence. In Proc. of COLT, 2008.

[26] P. Comon and C. Jutten, editors. Handbook of Blind Source Separation. Academic Press, 2010.

[27] P. Comon and M. Rajih. Blind identification of under-determined mixtures based on the characteristic function. Signal Processing, 86(9):2271-2281, 2006.

[28] S. Dasgupta. Learning mixtures of Gaussians. In Foundations of Computer Science, 1999. 40th Annual Symposium on, pages 634-644. IEEE, 1999.

[29] S. Dasgupta and A. Gupta. An elementary proof of a theorem of johnson and lindenstrauss. Random Structures and Algorithms, 22(1):60-65, 2003.

[30] S. Dasgupta and L. Schulman. A probabilistic analysis of EM for mixtures of separated, spherical Gaussians. The Journal of Machine Learning Research, 8:203-226, 2007.

[31] C. Davis and W. M. Kahan. The rotation of eigenvectors by a perturbation III. SIAM Journal on Numerical Analysis, 7(1):1-46, 1970.

[32] L. De Lathauwer, J. Castaing, and J. Cardoso. Fourth-order cumulant-based blind identification of underdetermined mixtures. Signal Processing, IEEE Transactions on, 55(6):2965-2973, 2007.

[33] L. De Lathauwer, B. De Moor, and J. Vandewalle. On the best rank-1 and rank- $\left(R_{1}, R_{2}, \ldots, R_{n}\right)$ approximation of higher-order tensors. SIAM Journal on Matrix Analysis and Applications, 21(4):1324$1342,2000$.

[34] S. C. Eisenstat and I. C. Ipsen. Relative perturbation results for eigenvalues and eigenvectors of diagonalisable matrices. BIT Numerical Mathematics, 38(3):502-509, 1998.

[35] A. M. Frieze, M. Jerrum, and R. Kannan. Learning linear transformations. In FOCS, pages 359-368, 1996.

[36] G. H. Golub and C. F. V. Loan. Matrix Computations. The Johns Hopkins University Press, 2013.

[37] R. A. Harshman. Foundations of the PARAFAC procedure: models and conditions for an "explanatory" multimodal factor analysis. 1970.

[38] C. Hillar and L.-H. Lim. Most tensor problems are NP-hard. Journal of the ACM, 60, 2013.

[39] D. Hsu and S. M. Kakade. Learning mixtures of spherical Gaussians: moment methods and spectral decompositions. In ITCS, pages 11-20, 2013.

[40] D. Hsu, S. M. Kakade, and T. Zhang. A spectral algorithm for learning hidden Markov models. 2009.

[41] A. Hyvärinen, J. Karhunen, and E. Oja. Independent Component Analysis. Wiley, 2001.

[42] A. T. Kalai, A. Moitra, and G. Valiant. Efficiently learning mixtures of two Gaussians. In Proceedings of the 42nd ACM symposium on Theory of computing, pages 553-562. ACM, 2010. 
[43] R. Kannan and S. Vempala. Spectral algorithms. Now Publishers Inc, 2009.

[44] T. G. Kolda and B. W. Bader. Tensor decompositions and applications. SIAM Review, 51:455-500, 2009.

[45] J. B. Kruskal. Three-way arrays: rank and uniqueness of trilinear decompositions, with application to arithmetic complexity and statistics. Linear algebra and its applications, 18(2):95-138, 1977.

[46] S. Lang. Complex analysis, volume 103. Springer, 1998.

[47] S. Lang. Undergraduate algebra. Springer Verlag, 2005.

[48] S. E. Leurgans, R. T. Ross, and R. B. Abel. A decomposition for 3-way arrays. SIAM Journal on Matrix Analysis and Applications, 14:1064-1083, 1993.

[49] L. Lovász and S. Vempala. The geometry of logconcave functions and sampling algorithms. Random Struct. Algorithms, 30(3):307-358, 2007.

[50] J. Marcinkiewicz. Sur une propriété de la loi de Gauss. Mathematische Zeitschrift, 44(1):612-618, 1939.

[51] A. Moitra and G. Valiant. Settling the polynomial learnability of mixtures of Gaussians. In Foundations of Computer Science (FOCS), 2010 51st Annual IEEE Symposium on, pages 93-102. IEEE, 2010.

[52] E. Mossel, R. O'Donnell, and K. Oleszkiewicz. Noise stability of functions with low influences: Invariance and optimality. Annals of Math., 171:295-341, 2010.

[53] E. Mossel and S. Roch. Learning nonsingular phylogenies and hidden markov models. In STOC, pages $366-375,2005$.

[54] P. Q. Nguyen and O. Regev. Learning a parallelepiped: Cryptanalysis of GGH and NTRU signatures. J. Cryptology, 22(2):139-160, 2009.

[55] K. Pearson. On lines and planes of closest fit to systems of points in space. The London, Edinburgh, and Dublin Philosophical Magazine and Journal of Science, 2(11):559-572, 1901.

[56] W. H. Press, S. A. Teukolsky, W. T. Vetterling, and B. P. Flannery. Numerical recipes 3rd edition: The art of scientific computing. Cambridge university press, 2007.

[57] M. Rudelson. Random vectors in the isotropic position. J. of Functional Analysis, 164:60-72, 1999.

[58] A. Sanjeev and R. Kannan. Learning mixtures of arbitrary Gaussians. In Proceedings of the thirty-third annual ACM symposium on Theory of computing, pages 247-257. ACM, 2001.

[59] A. Smilde, R. Bro, and P. Geladi. Multi-way analysis: applications in the chemical sciences. Wiley. com, 2005.

[60] N. Srivastava and R. Vershynin. Covariance estimates for distributions with $2+\epsilon$ moments. Annals of Probability, to appear (arXiv:1106.2775), 2011.

[61] J. M. Steele. The Cauchy-Schwarz Master Class. Cambridge University Press, 2004.

[62] G. W. Stewart and J.-g. Sun. Matrix perturbation theory. 1990.

[63] R. C. Thompson. Principal submatrices IX: Interlacing inequalities for singular values of submatrices. Linear Algebra and its Applications, 5:1-12, 1972.

[64] S. Vempala and G. Wang. A spectral algorithm for learning mixture models. Journal of Computer and System Sciences, 68(4):841-860, 2004.

[65] S. S. Vempala and Y. Xiao. Structure from local optima: Learning subspace juntas via higher order PCA. CoRR, abs/1108.3329, 2011. 
[66] R. Vershynin. Introduction to the non-asymptotic analysis of random matrices. In Y. Eldar and G. Kutyniok, editors, Compressed Sensing, Theory and Applications, pages 210-268. Cambridge University Press, Oxford.

[67] R. Vershynin. How close is the sample covariance to the actual covariance matrix? Journal of Theoretical Probability, to appear (arXiv:1004.3484), 2010.

[68] J. H. Wilkinson. The algebraic eigenvalue problem, volume 155. Oxford Univ Press, 1965.

[69] A. Yeredor. Blind source separation via the second characteristic function. Signal Processing, 80(5):897$902,2000$. 